\title{
III-V Ternary Nanowires on Si Substrates: Growth, Characterization and Device Applications
}

\author{
Giorgos Boras, Xuezhe Yu, ${ }^{*}$ Huiyun Liu \\ Department of Electronic and Electrical Engineering, University College London, London, \\ WC1E 7JE, United Kingdom
}

\begin{abstract}
Over the past decades, the progress in the growth of materials which can be applied to cutting-edge technologies in the field of electronics, optoelectronics and energy harvesting has been remarkable. Among the various materials, group III-V semiconductors are of particular interest and have been widely investigated due to their excellent optical properties and high carrier mobility. However, the integration of III-V structures as light sources and numerous other optical components on $\mathrm{Si}$, which is the foundation for most optoelectronic and electronic integrated circuits, has been hindered by the large lattice mismatch between these compounds. This mismatch results in substantial amounts of strain and degradation of the performance of the devices. Nanowires (NWs) are unique nanostructures that induce elastic strain relaxation, allowing for the monolithic integration of III-V semiconductors on the cheap and mature Si platform. A technique that ensures flexibility and freedom in the design of NW structures is the growth of ternary III-V NWs, which offer a tuneable frame of optical characteristics, merely by adjusting their nominal composition. In this review, we will focus on the recent progress in the growth of ternary III-V NWs on Si substrates. After analysing the growth mechanisms that are being employed and describing the effect of strain in the NW growth, we will thoroughly inspect the available literature and present the growth methods, characterization and optical measurements of each of the III-V ternary alloys that have been demonstrated. The different properties and special treatments required for each of these material platforms are also discussed. Moreover, we will present the results from the works on device fabrication, including lasers, solar cells, water splitting devices, photodetectors and FETs, where ternary III-V NWs were used as building blocks. Through the current paper, we exhibit the up-to-date state in this field of research and summarize the important accomplishments of the past few years.
\end{abstract}

Keywords: Ternary Alloys, III-V Nanowires, Si Substrates, Growth, Devices 


\section{1) Introduction}

The application of electronic and optoelectronic technologies in numerous aspects of daily life has been growing immensely over the past decades. The underlying platform on which most of the electronic and optoelectronic circuits and devices are founded is Si due to its low cost, good thermal conductivity, abundant availability and high-level maturity. ${ }^{[1]}$ On the other hand, group III-V semiconductors are particularly advantageous for optoelectronic devices, owing to the optical-active characteristics, which benefit optical applications including lasers, ${ }^{[2-3]}$ light emitting diodes (LEDs) ${ }^{[4-5]}$ and energy harvesting devices such as solar cells, ${ }^{[6-7]}$ while another equally important feature is the small carrier mass and the consequent high carrier mobility, rendering them ideal compounds for highspeed FET fabrication. ${ }^{[8-9]}$ Ergo, III-V on-chip integration with Si would enable the fabrication of circuits based on this material platform, which can provide the vital light source for Si photonics. Nevertheless, group III-V semiconductors exhibit a significant lattice mismatch with Si thus their combination induces a large amount of strain, which in turn causes severe problems such as the formation of threading dislocations that penetrate the III-V films and hinder the performance of the devices based on these materials. ${ }^{[10]}$ As a result, techniques that enable the integration of III-V semiconductors with $\mathrm{Si}$ are in high demand but challenging.

A solution that has been suggested is employing a sophisticated growth technique of buffer layer between the Si substrate and the III-V structure. ${ }^{[11]}$ Several materials have been attempted as buffer layers for the growth of III-V structures on $\mathrm{Si}$, the most popular of which are $\mathrm{GaAs},{ }^{[12]} \mathrm{Ge},{ }^{[13]} \mathrm{GeSi}^{[14]}$ and $\mathrm{GaP},{ }^{[15]}$ while $\mathrm{InGaP} / \mathrm{GaP}^{[16]}$ and $\mathrm{GaAsP} / \mathrm{GaP}^{[17]}$ buffer layers with composition gradient have also been used. Another method that has been developed is the non-epitaxial growth of $\mathrm{GaAs}$ on $\mathrm{Si}$, via the so-called 'wafer bonding', which includes the attachment of bulk or thin film III-V semiconductors on Si substrates. This technique can be implemented at room temperature, assuming that the surface roughness is minimized and the surfaces are flat and ideally clean. ${ }^{[11]}$ With the careful operation of the bonding procedure, the surfaces are bonded to each other via van der Waals forces. ${ }^{[18]}$

A novel method to achieve III-V growth on Si is to employ semiconductor nanowires (NWs), an emerging and promising platform that is being thoroughly investigated and rapidly developed mainly during the past two decades. The small contact area between the substrate and the NW along with the large surface to volume ratio leads to elastic strain relaxation, which allows for the monolithic growth of III-V NWs on Si substrates and thus renders the direct integration of III-V semiconductors on the Si platform feasible. ${ }^{[19]}$ Due to this high strain tolerance, the buffer layer, which requires sophisticated growth techniques, is no longer necessary.

An essential aspect of NWs research that is being under substantial investigations and is employed for applications is the use of ternary NWs, where, for III-V semiconductors, two group III and one group V or two group V and one group III elements are combined. This offers a high degree of freedom 
in material design owing to their fully tuneable bandgap depending on the elemental composition of the alloy. Generally, the elemental composition of the alloy is determined by the growth conditions and growth methods that are used and can be tuned to great extents by altering the growth parameters. Due to this flexibility that can be approached via the optimization of the growth, ternary NWs have been implemented in multiple applications such as energy harvesting, for example, solar cells ${ }^{[20-21]}$ and water splitting devices. ${ }^{[22]}$ Moreover, they have been used for the realization of lasers ${ }^{[23-24]}$ and LEDs ${ }^{[25-26]}$ with a controlled emission, which is determined by the rational design of the composition of the alloy. The tuneable bandgap is also crucial for ternary NW-based photodetectors with comparable or even enhanced figures of merit when compared to devices based on binary NWs. ${ }^{[27-28]}$ Another benefit in some ternary NWs is that the addition of an element can significantly improve the material quality, by reducing the number of defects. A specific category of these NWs is antimonides that promote a pure ZB crystal phase, due to the surfactant properties of $\mathrm{Sb}^{\left[{ }^{[29-30]}\right.}$ This factor is of importance, as crystal purity can enhance the intensity of radiative recombination and eliminate losses in the devices. Finally, the high carrier mobility of group III-V semiconductors indicates an extra advantage of III-V ternary NWs for high-speed FETs, which exhibit optimal electron transport properties. ${ }^{[31-32]}$

In this review, we will present the recent advances in the growth and characterization of ternary III$\mathrm{V}$ NWs grown on Si substrates. By examining the rich literature on this vibrant field, we have summarized each III-V ternary alloys that are available in the literature in terms of the growth methods, characterization and optical properties of each ternary alloy. In the first section, we will briefly discuss the different growth mechanisms, namely the vapour-liquid-solid (VLS) growth mode, selective area growth (SAG) and catalyst-free technique and the characteristics of each of them. Then, we will describe the effect of the strain for the growth of axial heterostructures or core/shell ternary III-V NWs, which is inevitably considered due to the essential of the heterostructure growth. A thorough description of each of the ternary alloy NWs will be demonstrated in the third section, including GaAsP, AlGaAs, GaAsSb, InAsSb, InGaAs and InGaP. Particular emphasis will be given to the aspects of the growth, the crystal purity of the NWs, as well as to the optical and electrical properties of the NWs. For some of these cases, growth on substrates other than $\mathrm{Si}$ is briefly included for comparison or for supplementary information, which includes the description of the growth of four rare III-V alloys of InGaSb, GaPSb, InPSb and AlInP, in the third section as well. Finally, we will briefly describe the various devices that have been implemented on the ternary III-V, such as lasers, solar cells, photodetectors, water splitting and FETs and their performance and potential optimizations are discussed. 


\section{2) Growth mechanisms}

Ternary III-V NWs can be grown via epitaxial techniques available such as Molecular Beam Epitaxy (MBE), Metal-Organic Vapour Phase Epitaxy (MOVPE) also known as Metal-Organic Chemical Vapour Deposition (MOCVD), Chemical Beam Epitaxy (CBE) and Chemical Vapour Deposition (CVD). In each of the growth systems, there are two major growth modes that are being used extensively, namely the selective-area growth (SAG) and the vapour-liquid-solid (VLS) growth mode. The progress on these fundamental growth modes for ternary NW growth is remarkable. Nevertheless, there are still several challenges met in each of them and their exact solution has not been fully unravelled yet.

SAG for ternary NWs is a growth method where the NW growth occurs through holes patterned on the substrate by the use of a mask and a lithography technique (i.e. nanoimprint lithography, electron beam lithography (EBL) etc. ${ }^{[33]}$ SAG has been frequently employed for NW growth, with the most prominent advantage being the high level of homogeneity in the position and the size distribution of the NWs, leading to the realization of highly uniform NW arrays (Figs. 1) ${ }^{[34]}$ Furthermore, the lack of a catalyst droplet leads to the benefit of avoiding the reservoir effect, which can cause non-abrupt interfaces between different sections of the NW structure. The reservoir effect will be further described later. The principles that lie beneath SAG are similar to the ones in thin film epitaxy. In this case, however, the use of a patterned pitch on the substrate suppressed growth in the lateral dimensions and allows only for perpendicular elongation of the NW. Despite this fact, parasitic growth may occur, which can hinder the applicability of the NWs in large scale. It is noted that this challenge is encountered for the rest of the growth techniques, as well. Additionally, the common use of lithography techniques such as EBL greatly enhance the cost of the growth, making SAG an economically unfavored growth method ${ }^{[35]}$ Despite these challenges, the position controlled merit of SAG has led to many advances over the past years. NW arrays grown via SAG have been successfully used for device fabrication, such as the surround-gated FETs ${ }^{[36]}$ and LEDs. ${ }^{[37]}$
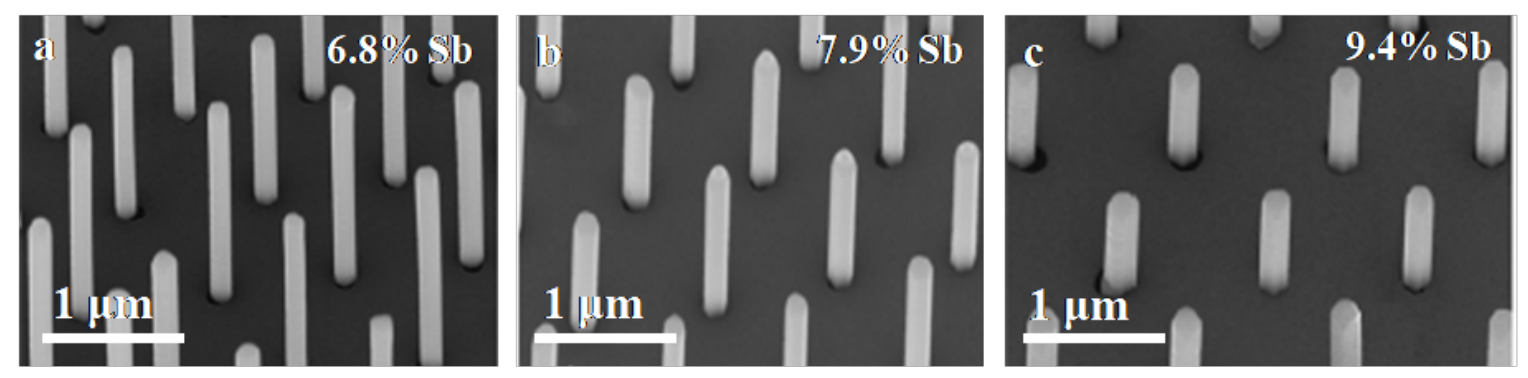

Figure 1: a-c) Scanning Electron Microscopy (SEM) images of InAsSb/GaSb core/shell NWs grown via MOCVD following SAG mode. The arrays of the grown NWs have a high level of homogeneity. NWs 
were grown with different Sb compositions, as indicated in each image. (Figs. 1a-c: Reprinted with permission from ref. 34. Copyright (2016) American Chemical Society)

VLS growth is the most popular method for the growth of ternary NWs. The growth is induced by a catalyst droplet, which can be either a foreign metal seed or an element which is also a component of the ternary NW. For ternary NWs of group III-V, group III elements can be used, for instance, Ga and In. Al can also be used as a catalyst droplet, ${ }^{[38]}$ even though there are no current reports on its use for the growth of III-V ternary NWs. VLS for III-V NWs can be, thus, subcategorized in two groups, extrinsic metal-assisted growth and intrinsic-metal-catalysed growth. In extrinsic metal-assisted growth, the growth is initiated by metal droplets (most usually $\mathrm{Au}$ ) which act as the collector, while adatoms introduced in the reactor are the suppliers. ${ }^{[39]}$ To form the metal droplets, metal nanoparticles are deposited on the substrate, which are liquefied via thermal annealing (Fig. 2a). After the group III or group V adatoms reach the droplet, they form a liquid alloy with it. With the continuous supply of the adatoms, this alloy becomes supersaturated and in order for the chemical balance to be restored, an island nucleates at the liquid/solid interface which rapidly expands and forms a monolayer covering the entire interface and the NWs grow axially (Fig. 2b) ${ }^{[39]}$ There are two main paths for the group III-V adatoms to reach the droplet, either via direct impingement or by diffusion over the substrate and the NW sidewalls (Fig. 2c). The growth is terminated after the sources are not supplied any more in the reactor (Fig. 2d). It is noted that group V atoms, have a low solubility in Au and need to be continuously supplied with a sufficiently high flux in the reactor to reach the droplet and maintain the amount of group $\mathrm{V}$ adatoms soluted in the droplet for the axial elongation of the $\mathrm{NW} \cdot{ }^{[10]} \mathrm{Au}$ is suggested to be superior to other metal seeds because of some of its properties, including the thermal stability, chemical inertness and the fact that most used alloys are soluble in $\mathrm{Au}^{[40]}$ Despite its advantageous properties, the presence of $\mathrm{Au}$ introduces some difficulties in the growth and applicability of the structures. First of all, it can diffuse into the NW and form deep trap levels that deteriorate the optical and electrical performance of the structures. ${ }^{[41]}$ Additionally, it can be alloyed with the Si substrates and form midgap states, a phenomenon which is detrimental as it renders the NWs incompatible with CMOS technology applications. ${ }^{[41]} \mathrm{Au}$ is also hard to remove and is considered a significant contaminant for the growth system. Another difficulty is the reservoir effect, according to which some elements are not entirely consumed in the Au droplet. As a result, they can diffuse and incorporate in the NW even after the corresponding supply of elements is terminated. Therefore the abruptness of the interfaces between the different sections of the NWs is jeopardized. ${ }^{[42]}$ Alternatively, other metals, such as Ag has also been employed as a catalyst for the growth of InGaAs NWs via MOVPE. ${ }^{[43]}$ However, the metalcatalysed growth with alternative metal seeds other than $\mathrm{Au}$ is far from being understood. 


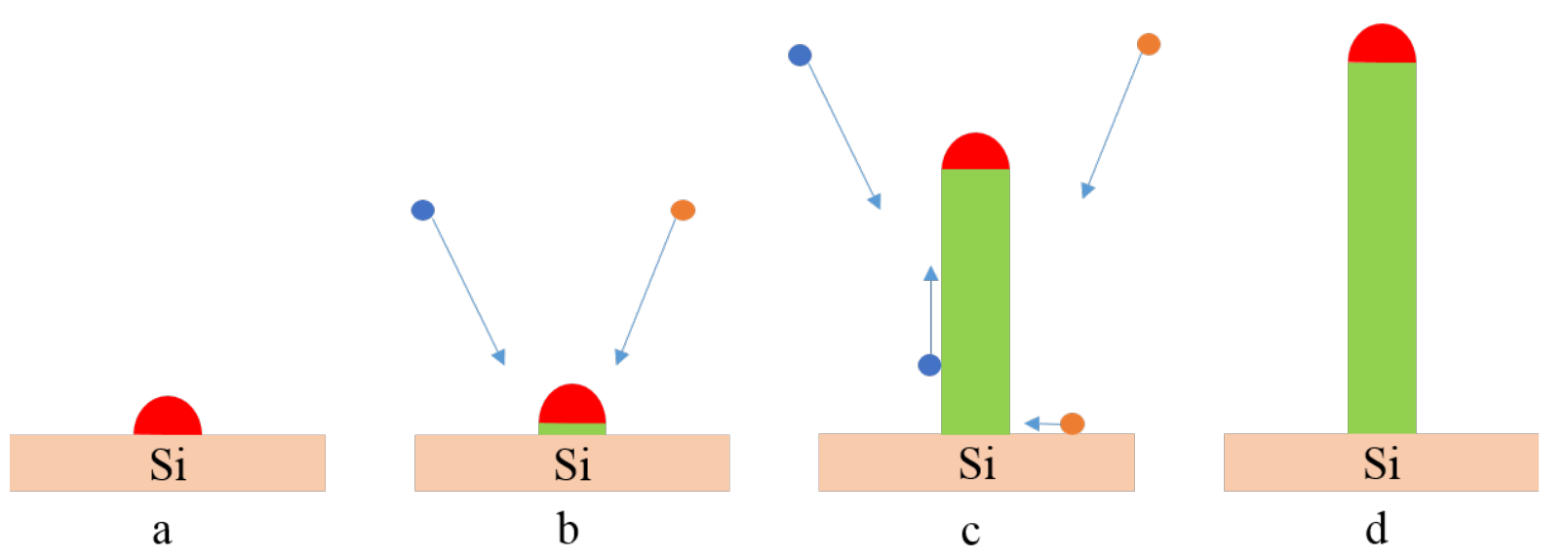

Figure 2: a) Droplet is deposited on the Si substrate. b) Elements are supplied in the reactor. The adatoms are alloyed with the liquid droplet and, after supersaturation, a monolayer is formed covering the liquid/solid interface. c) The continuous supply of elements leads to the elongation of the NW. The adatoms reach the droplet either via direct impingement or after diffusion on the substrate and the NW sidewalls. d) After the supply of elements is terminated, the axial growth stops.

Due to the detrimental effects of Au on the applicability of the NWs, the need for an alternative route was emerging. This route could be the self-catalysed growth, where catalyst droplets that initiate the growth are also one of the components of the ternary NW. The growth mode is similar to the case of the foreign metal catalyst. The fact that the material of the droplet is already a component of the NW eliminates the contamination, as mentioned earlier. ${ }^{[44]}$ Apart from the different catalyst droplet, the growth procedure is similar to the Au-catalysed growth (Fig. 2b-d). It is noted that the growth of IIIIII-V ternary alloys is considered much more challenging via the self-catalysed VLS growth mode when compared to the growth of III-V-V alloys. It is due to the high solubility of group III atoms in the droplet, any alterations between group III elements would signify substantial chemical differentiation in the droplet and even modify the chemical and physical properties of droplet itself since it mainly consists of group III atoms. VLS growth of ternary NWs is rendered challenging due to the commonly observed inhomogeneous elemental distribution along the entire NW structures in both self-catalysed and Au-catalysed approaches. ${ }^{[45-46]}$ Despite its complexity and sensitivity, the self-catalysed VLS growth mode is compatible with MBE well, which is one of the most advanced epitaxial tools and can be employed for the growth of sophisticated structures. As a result, large amounts of effort have been devoted to fully unravelling its mechanism and potential in the field of MBE research.

As stated earlier, the reservoir effect which appears in VLS growth of ternary NWs hinders the abruptness of the interfaces. ${ }^{[42,47]}$ Another alternative that is currently under investigation is the catalystfree mode. In catalyst-free mode, no droplet initiates the growth of the NW. ${ }^{[48-49]}$ The driving force of the NW growth, in this case, could be the surface roughness of the substrate that affects the kinetics of the materials or strain, which is induced by a significant lattice mismatch between the NW compounds and the substrate. ${ }^{[50]}$ That way, the NW is grown without any contamination and non-abrupt interfaces. 
Despite its advantages, this growth technique is not entirely understood yet, and the literature based on the catalyst-free approach is relatively less when compared to SAG or VLS growth.

Ternary III-V NWs have been grown via all three of the described techniques. It is highlighted, however, that due to the disadvantages of the Au droplet, self-catalysed and catalyst-free methods are particularly suitable for the growth of ternary NWs on Si substrates. In the next chapter, we will briefly describe the role of strain in NW structures, before continuing with the central part of our literature review.

\section{3) Effect of strain}

The integration of group III-V semiconductor structures with the cost-effective and mature $\mathrm{Si}$ platform has been hindered by the significant lattice mismatch between them, which induces high amounts of strain. The existence of strain would give rise to defects such as misfit dislocations and more importantly, threading dislocations that are formed in III-V structures. Threading dislocations are considered non-radiative recombination centres, which severely affect the intensity of the PL emission, thus degrading the performance of the resulting optoelectronic device. Other defects that may emerge include stacking faults, rotational twins, and grain boundaries. Due to the profound influence that these crystal defects have on the performance of the structures, intense efforts have been devoted to the optimization of their material quality and consequently, their optical performance.

NWs are unique compared with other nanostructures or thin films because of their large strain tolerance. Owing to their geometrical features, such as the large surface to volume ratio and the small contact area between the NW and the substrate, there is a critical radius below which the strain is elastically relaxed. ${ }^{[51]}$ This enables the monolithic integration of group III-V NWs on the mature Si platform and ternary NWs on binary NW stems. Notably, the latter is extensively applied in cases where monolithic growth of NWs on Si is impractical and allows for the NWs to adopt unique properties and features. ${ }^{[52]}$

As a result of the above, it is clear that axial NWs are free of strain. It is noted that misfit dislocations can still be observed at the NW/substrate interface. ${ }^{[33-55]}$ Nevertheless, threading dislocations are commonly absent in the case of axial NWs. That is not the case, however, in core/shell NWs. The strain induced by the lattice mismatch between the core and the shell is relaxed inelastically, with potential formation of dislocations between the lattice-mismatched core and shell. ${ }^{[56-57]}$ Strain residues can still alter the morphology and properties of NWs in many cases. An example of this phenomenon is the occasional bending and kinking of NWs in the presence of some non-negligible amounts of strain attributed to the formation of a lopsided shell surrounding the NW cores. ${ }^{[58-59]}$ There are mainly two types of strain in epitaxial growth, namely tensile and compressive strain. It is noted that the case of tensile-strained NW core has been examined in the past. ${ }^{[58-59]}$ After an initial bending occurring due to the strain, adatom incorporation is preferential at the convex side, leading to the NW bending towards 
the thinner side of the shell (Figs. 3a-b). ${ }^{[59]}$ The behaviour of the compressively-strained core should be opposite. Unfortunately, the existing literature on compressively strained core NWs is much more limited and as a result, the anticipated behaviour cannot be distinctly observed. ${ }^{[60]}$ This indicates that there is the need for further investigations regarding this topic to unambiguously confirm the different distortions induced under the influence of compressive and tensile strain. The vital role of strain in the properties of core/shell NWs along with the complexity regarding its relaxation has led to several works being conducted for the evaluation and calculation of strain in these structures. ${ }^{[61-63]}$
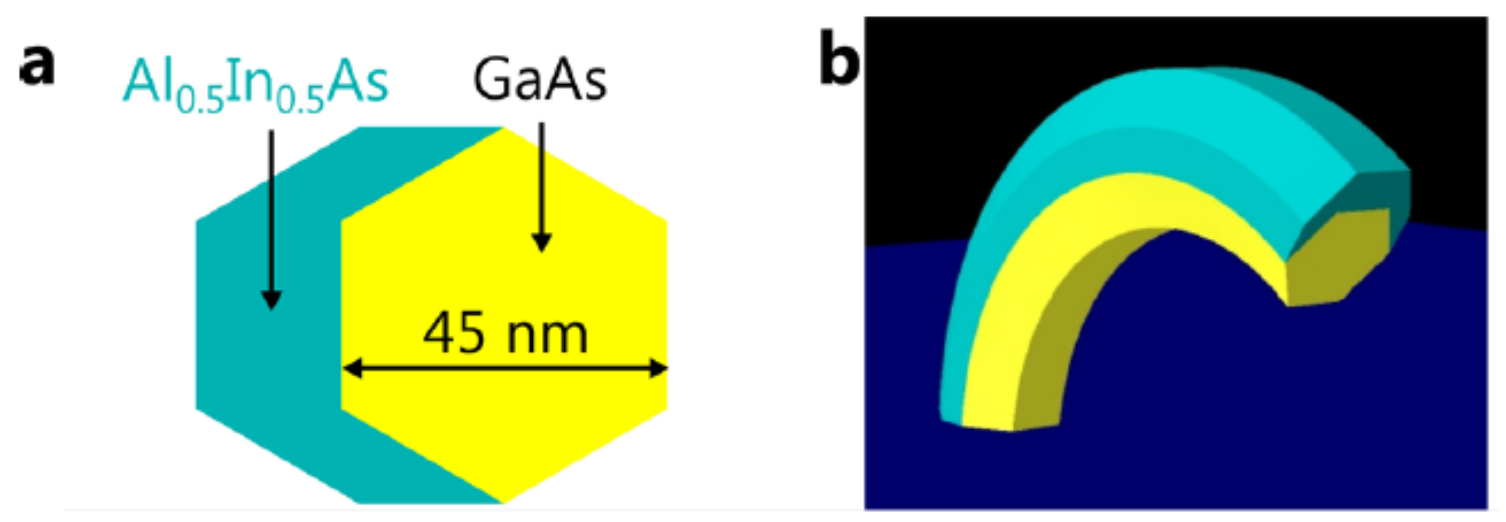

Figure 3: a-b) Top view and front view schematic representations respectively for a GaAs NW cladded with a $20 \mathrm{~nm}$ thick AlInAs shell. The strain that is induced by the lattice mismatch between the core and the shell causes a severe bending of the NW. The convex side is more appropriate for adatom accommodation, further increasing this phenomenon. (Figs. 3a-b: Reprinted with permission from ref.

\section{Copyright (2018) American Chemical Society)}

Based on the above results found in the literature, the strain can give rise to detrimental effects on the core/shell NW morphology and composition. A direct path to circumvent this obstacle is the combination of lattice-matched materials in core/shell structures. The minimum amount of strain between the core and the shell has been exploited to realize not only III-V core/shell NWs but even hybrid III-V/IV NW structures, with vast potential for optoelectronic applications. ${ }^{[64]}$ Nevertheless, this solution limits the possible alloys that can be combined.

Despite the types of strain that can be induced in the core/shell structures or intentionally applied in the NWs, the unique axial NW geometry allows for efficient, elastic strain relaxation which in turn enables compatibility of ternary III-V NWs with the mature Si platform. That property renders the NWs particularly appealing for a wide range of applications, where Si plays a crucial role such as electronics, optoelectronics and solar cells. Due to the effective elastic relaxation, strain plays a less significant role in the growth of axial NWs. The detailed investigation of core/shell architecture and the effects of strain is beyond the scope of this review. In the rest of the current report, we will focus on the growth and analysis of NWs with ternary cores. 
In the following section, we will investigate the rich literature of each type of NWs that has been successfully grown, and some of them have already been applied to the prototypical devices. Particular emphasis will be given to the different aspects of the growth and to the characterization of NWs regarding their crystal structure to conclude on their material quality. A description of the optical and electrical properties of the ternary NWs will also be included. These features enable us to evaluate the suitability of these nanostructures for the fabrication of devices, which will be later analysed in the fifth section. Through this procedure the growth, characterization and device performance of ternary NWs will be discussed and clarified.

\section{4) Ternary III-V NWs on Si}

\section{1) III-V-V Nanowires}

\subsection{1) GaAsP Nanowires}

One of the most explored ternary NWs is GaAsP. The interest in this alloy is mainly due to the considerable frame of tunability of its bandgap, which can theoretically range between $1.43 \mathrm{eV}$ (GaAs) and $2.24 \mathrm{eV}(\mathrm{GaP})$ at room temperature. Even though $\mathrm{GaP}$, as one of its edge alloys, has an indirect bandgap, GaAsP exhibits an indirect to direct transition at $44 \% \mathrm{P}$ content, ${ }^{[65]}$ which has an ideal band alignment for water splitting devices and a chemical stability that allows it to be used as a photocathode, ${ }^{[22]}$ rendering it ideal for energy applications such as tandem solar cells on Si. It is interesting to mention that the growth of $\mathrm{GaP}$ on $\mathrm{Si}$ has been realized, which signifies that the entire coverage of the bandgap range between GaAs and GaP is possible. ${ }^{[66]}$ Furthermore, GaP can have a direct bandgap once crystallized into the WZ phase, which has already been realized on ZB GaP substrates successfully. ${ }^{[67]}$ As a result, it can be safely assumed that obtaining WZ GaP on Si substrates would allow the emission coverage reaching a wide range from the green to the near-infrared region of the spectrum. The growth of GaAsP NWs has been achieved via different epitaxy techniques such as Metal-Organic Vapour Phase Epitaxy (MOVPE) ${ }^{[68]}$ and Molecular Beam Epitaxy (MBE). ${ }^{[22,69-71]}$

One of the most crucial tasks during the growth of ternary NWs is defining the appropriate parameters that will determine the morphological features and the consequent properties of the structures. Among the growth parameters, the most critical one in ternary NW growth is the V/III ratio, owing to the different incorporation rates of group III and group $\mathrm{V}$ adatoms. By altering the V/III ratio, the morphology of the NWs can be entirely altered from uniform, standing NWs to tapered or cluster-like structures, due to changes in the geometry of the Ga droplets that initiate the growth. In the case of GaAsP NWs, at a relatively low V/III beam flux ratios of 50, the droplets are fully formed and clearly define the boundaries for the growth of the NWs (Fig. 4a). ${ }^{[69]}$ As a direct result, most of the resulting NWs are standing on the Si substrate, with a high degree of homogeneity. With increasing V/III ratio 
at 75 , the droplets shrink during growth which causes a severe tapering at the NW tip (Fig. $4 b$ ). Eventually, at an excessive V/III ratio of 100 , the group V flux is so much increased that the droplets are rapidly consumed and no VLS growth takes place (Fig. $4 \mathrm{c}$ ). ${ }^{[69]}$ Changes in the V/III ratio also affect the crystal structure of the NWs. GaAsP NWs grown under optimized V/III ratio adopt a pure ZB crystal phase in their middle part. It is interesting to observe that the NW tip and base exhibit defects (Fig. 4d), because of the high group V flux and instability of the growth, respectively. ${ }^{[69]}$ On the contrary, GaAsP NWs grown under enhanced V/III ratios exhibit a polytypic nature and are crystallized into the mixed WZ/ZB phase (Fig. 4e). ${ }^{[69]}$ This differentiation in the crystal structure of the NWs has a profound effect on their optical properties, as well.

The phosphoric content of GaAsP NWs is of great importance for the optical properties of the structures and is directly related to the bandgap of GaAsP. The UV visible diffuse reflectance spectrum presented in Fig. $4 \mathrm{f}$ analyses the photon absorption for NWs with a different nominal composition of $\mathrm{P}$. The onset value of the absorption band is determined as the value of the bandgap of the semiconductor. ${ }^{[68]}$ Two NWs were measured with different thickness of $60 \mathrm{~nm}$ (dotted lines) and 120 $\mathrm{nm}$ (solid lines). It is noted that the tuning range of the bandgap was different, ranging between $1.48 \mathrm{eV}$ and $2.28 \mathrm{eV}$ for narrow NWs and $1.35 \mathrm{eV}$ and $2.23 \mathrm{eV}$ for wide NWs (Fig. 4g) thus it concludes that the bandgap of narrow NWs is larger than that of wide NWs. However, with increasing P content these two values approach each other. That is attributed to the narrower bandgap of the multiple WZ segments when compared to ZB segments, the presence of which is increasing at higher P content.

Most applications require a highly homogeneous array of NWs, which is particularly challenging, especially in the case of ternary NWs. A potential solution is the patterning of the Si substrates via lithography techniques. This method is costlier due to the additional processing steps but it is also instrumental in defining the exact position and dimensions of the NWs that form the array. The size of the holes is a vital parameter to be considered. When the size of the hole is equal or smaller than the diameter of the droplet, the latter covers the hole in its entirety, causing all of the Ga adatoms to reach the droplet and participate in the axial growth of the GaAsP NWs (Fig. 4h). On the contrary, when the size of the holes is larger than the diameter of the droplet, a vacant, oxide-free Si area is exposed and the Ga, $\mathrm{P}$ and As adatoms would cover this area and giving rise to lateral vapour-solid (VS) growth. That phenomenon reduces the number of $\mathrm{Ga}$ adatoms migrating to the droplet, leading to the formation of NWs with very narrow tips and mostly wide bases. ${ }^{[70]}$ In case the size of the hole is much larger than the diameter of the droplet, the aforementioned phenomenon is more pronounced and the NWs take the form of short nanopillars (Fig. 4i). ${ }^{[70]}$ 

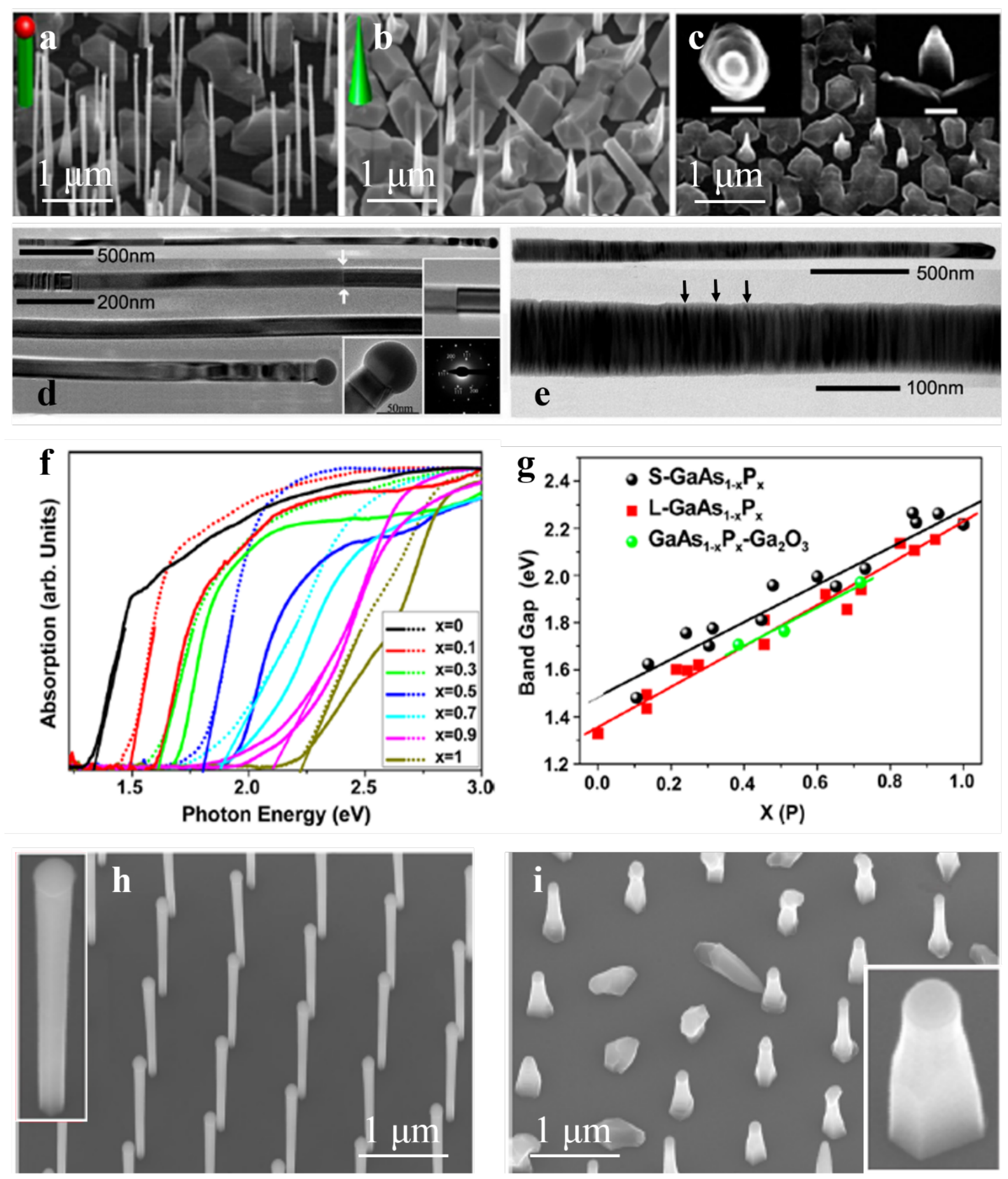

Figure 4: a) SEM image of GaAsP NWs grown under V/III ratio of 50. Uniformity and vertically aligned $N W$ s are observed. b) SEM image of GaAsP NWs grown under V/III ratio of 75. The growth rate is reduced due to shrinkage of the droplet, causing a severe tapering in the NWs. c) SEM image of GaAsP NWs grown under V/III ratio of 100. Large group V flux rapidly consumes the droplets and no VLS growth takes place. d) Transmission Electron Microscopy (TEM) image of the overall and section views of a NW grown under V/III ratio of 50. In the middle of the structure, no defects are observed and the crystal adopts a pure ZB phase. The only defects are located at the top and bottom parts of the NW. The 
SAED pattern, a higher magnification on the droplet and a higher magnification of the part indicated by the white arrows are given in the insets. e) A TEM image of a NW grown under V/III ratio of 75. Multiple defects are viewed in the image due to the increased flux of group V elements. The defects appear as regions of different brightness and three typical examples are shown with black arrows. (Figs. 4a-e: Reprinted with permission from ref. 69. Copyright (2013) American Chemical Society) f) UV visible diffuse reflectance spectrum (absorption) of both narrow NWs (dashed line) and wide NWs (solid line) at different $P$ contents. $g$ ) Dependence of the bandgap on the nominal composition of $P$, for narrow and wide NWs. (Figs. 4f-g: Reprinted with permission from ref. 68. Copyright (2014) American Chemical Society) h) SEM of NWs grown on patterned substrate, where the diameter of the holes is 50 $n m$ and is smaller than the diameter of the droplet. A highly homogeneous $N W$ arrays is formed. In the inset, a higher magnification of a single $N W$ is depicted, confirming the homogeneous shape of the structure. i) SEM image of NWs grown on patterned substrate where the diameter of the holes is 135 $\mathrm{nm}$. The promotion of lateral VS growth leads to the structures taking the form of short nanopillars, as viewed in the inset. (Figs. 4h-i: Reprinted with permission from ref. 70. Copyright (2014) American Chemical Society. Further permissions related to the material excerpted should be directed to the ACS.)

Interesting morphological and optical features can be observed after embedding a single GaAs QD axially in the GaAsP NWs. ${ }^{[71]}$ The resulting structures are a popular alternative for QD fabrication, allowing for a potentially enhanced intensity of the emission and optimum optical properties. In ref. 71, the presence of the QD is confirmed via the Transmission Electron Microscopy (TEM) image of Fig. 5a, where the brighter segment circled, reveals the higher As content of the dot. The above were also corroborated via Energy Dispersive X-ray (EDX) mapping along the length of the GaAsP NW (Fig. $5 b)$. The map of $\mathrm{P}$ presents a darker region, corresponding to a brighter region in the As map, illustrating the axial stacking of a narrow GaAs segment. Emission deriving from the dot is sharp and are located around $1.66 \mathrm{eV}$ (Fig. 5c). The linewidth is as narrow as $130 \mu \mathrm{eV}$. It is noted that carriers can still escape the QD region and recombine radiatively in the NW section, thus causing the appearance of a weaker GaAsP peak (Fig. 5c). ${ }^{[71]}$ 

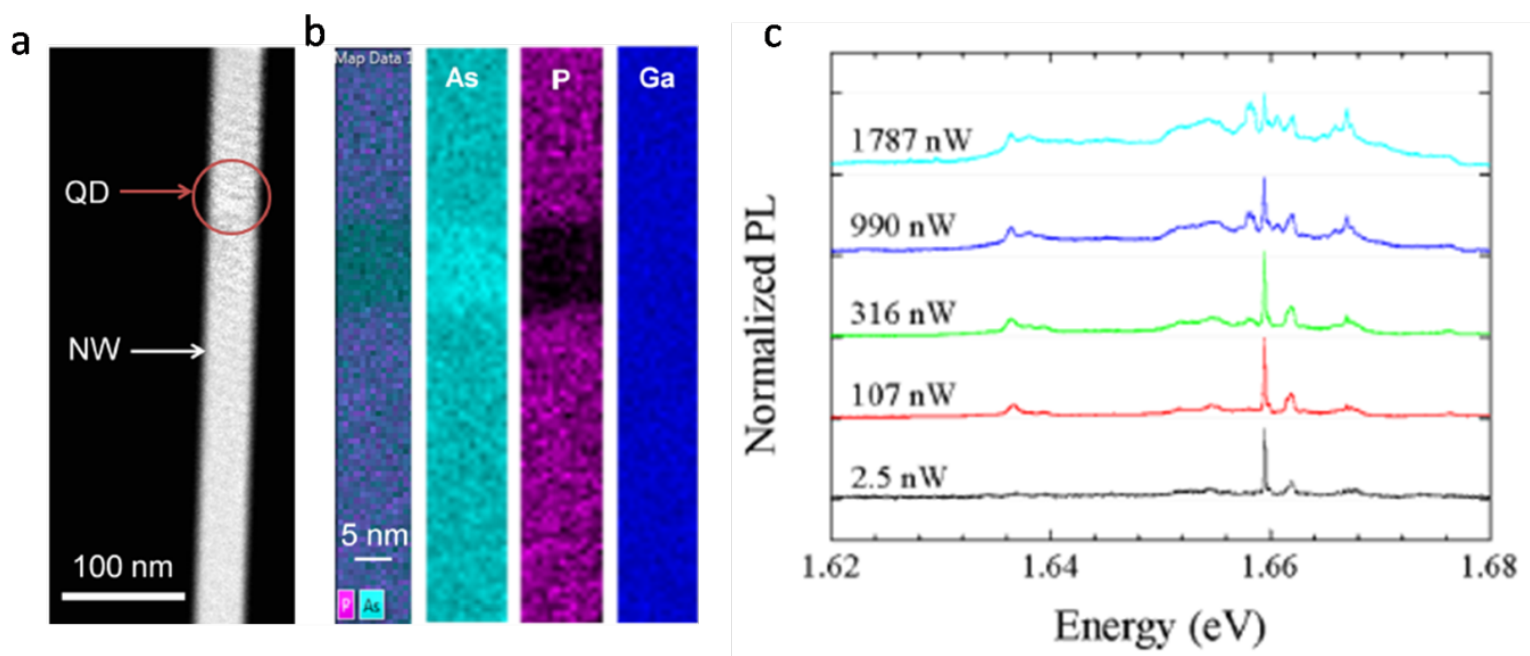

Figure 5: a) TEM image of a GaAs $Q D$ embedded in a GaAsP NW. The brighter region, revealing the presence of the dot, is marked by the red circle. b) EDX mapping of the structure. The QD section exhibits a higher As and lower P content than the NW part of NWQDs. c) Micro PL spectra at different excitation powers from a single GaAsP/GaAs NWQD. Sharp peaks attributed to $Q D$ emission are observed at $1.66 \mathrm{eV}$. The linewidth is measured as narrow as $130 \mu \mathrm{eV}$. (Figs. 5a-c: Reprinted with permission from ref. 71. Copyright (2015) American Chemical Society)

\subsection{2) InAsP Nanowires}

InAsP is a ternary alloy with an extensive range of tunability of its bandgap. The bandgap energy varies between 0.36 (InAs) and $1.344 \mathrm{eV}$ (InP), covering a full range from the near-infrared to the midinfrared regions of the spectrum. InAsP NWs also has high electron mobility and large absorption coefficient. Nevertheless, the growth of InAsP NWs on $\mathrm{Si}$ has been hindered by the highly inhomogeneous compositional distribution that derive from the VLS growth mode. The reports on InAsP NWs monolithically grown on $\mathrm{Si}$ are, indeed, limited. ${ }^{[21,72-73]}$ Consequently, in this section, we will also refer to InAsP NWs grown on different substrates as well, in order to expand our comprehension of their features and properties.

The growth of InAsP NWs on Si can be conducted via MOCVD ${ }^{[21,72]}$ or MBE. ${ }^{[73]}$ This material platform allows for a high level of uniformity of vertically aligned NWs, which is preferred for applications including optoelectronics and energy harvesting. A specific technique to achieve such a result is the treatment of the substrate by a poly-L-lysine (PLL) solution, before the growth. ${ }^{[21]}$ The addition of the PLL results in a polyelectrolyte layer on the Si wafers that creates a positive charge on the surface ${ }^{[21]}$ Even though PLL is a contaminant for the material system after its thermal annealing, its positive effect on the homogeneity of the standing NWs is noticeable after Scanning Electron Microscopy (SEM) measurements (Fig. 6a). A statistical analysis of the dimensions of the NWs in three 
different positions of the wafer (labelled A, B, and C) is presented in Fig. $6 \mathrm{~b}$ and corroborates the homogeneous distribution of the dimensions of the structures. These NWs are found to adopt a ZB structure, without any WZ segments being formed. Even so, a massive amount of stacking faults are still exhibited in the middle part of the structures (Fig. 6c). ${ }^{[21]}$ This large number of stacking faults is also confirmed by the streaky patterns in selected area electron diffraction (SAED) image as in the inset of Fig. 6c. A TEM inspection at the interface between the NW and the substrate shows a small number of misfit dislocations (Fig. 6d), owing to the lattice mismatch between InAsP and Si. However, from the SAED patterns of the inset of Fig. $6 \mathrm{~d}$ at the NW/Si interface, it is observed that bright spots appear, indicating purity in ZB crystal phase. ${ }^{[21]}$

On the contrary to $\mathrm{Si}$, some reports are focused on the growth on InAs (111)B substrates ${ }^{[74-75]}$ or InP (111)B substrates. ${ }^{[76-77]}$ In those cases, lattice mismatch between the NWs and the III-V substrates are less significant than that for $\mathrm{Si}$, which makes the growth more feasible. As a result, we consider it essential to include some results of InAsP growth on other substrates to obtain a better view of this alloy. Apart from the growth of InAsP NWs, another highly important structure that has been obtained is axial heterostructure, where InAsP segments are embedded in NWs comprised of a different material. That way the tuning of the compositional range is widened. ${ }^{[74-76]} \mathrm{A}$ characteristic example is the realization of InAsP segments embedded in InP NWs grown on InP (111)B substrates, ${ }^{[76-77]}$ which demonstrate the bright and narrow peaks of single photon emission and high light extraction efficiency. In the micro-PL spectrum measured at $10 \mathrm{~K}$, which is presented in Fig. 6e, two sharp peaks correspond to two InAsP QDs embedded in an InP NW. The peaks are very narrow with a linewidth of $120 \mu \mathrm{eV}$, as a result of the quantum confinement effect in the InAsP sections. ${ }^{[76]}$ In addition, via Au-assisted CBE growth on InAs (111)B substrates, the elemental composition of the ternary alloy segments could be tuned between $20 \%$ and $100 \% \mathrm{P}$ content. ${ }^{[7]}$ So although the growth of InAs/InAsP axial heterostructures, including NWQDs, has not been demonstrated on Si so far, all of the above results and their importance for applications in the field of electronics and optoelectronics render the attempt to monolithically grow these structures on the Si platform very promising. 

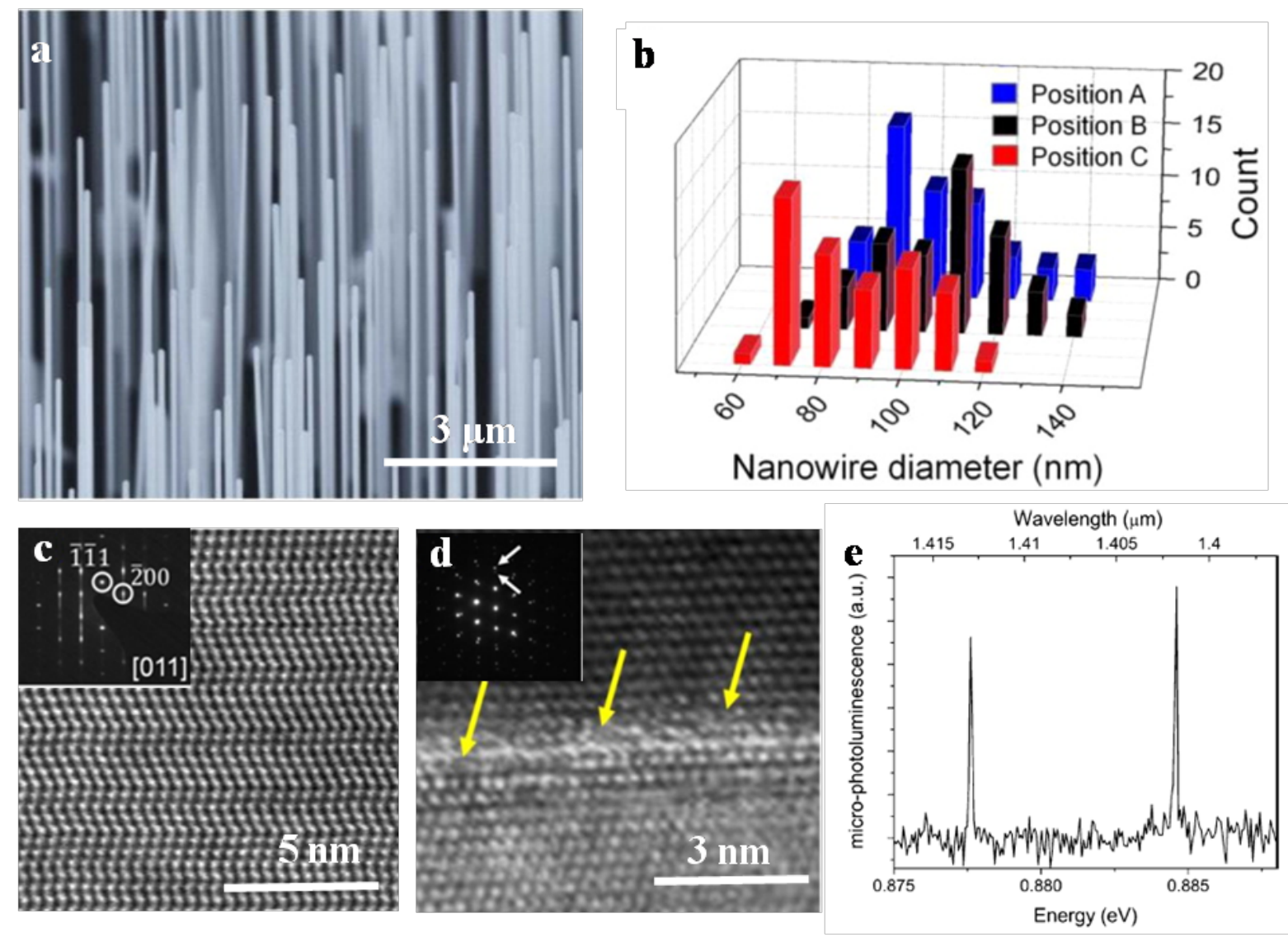

Figure 6: a) SEM image of InAsP NWs tilted by $45^{\circ}$. The NWs are perpendicular to the Si substrate and their density is estimated at $5.5 \times 10^{8} / \mathrm{cm}^{2}$. b) Diameter distribution respectively for the InAsP NWs. High uniformity is confirmed. c) High Resolution TEM (HRTEM) of the middle part of an InAsP NW. Multiple stacking faults are observed. The inset presents the SAED pattern of this part. The streaky features confirm the formation of stacking faults. d) HRTEM of the NW/Si interface. Misfit dislocations appear and are marked by yellow arrows in the image. In the inset the SAED pattern of the interface is shown. The clear spots reveal a pure ZB structure at the NW/Si interface, which leads to the conclusion of stacking faults being developed after the nucleation of the InAsP NWs at the initial stages of the growth. (Figs. 6a-d: Reprinted with permission from ref. 21. Copyright (2013) American Chemical Society) e) Micro-PL spectrum of a single InP NW with two InAsP QD insertions. The peaks corresponding to the QDs are located at $1.401 \mu \mathrm{m}$ and $1.412 \mu \mathrm{m}$ and are very sharp and narrow with a full width at half maximum of $120 \mu \mathrm{eV}$. The narrow peaks are indicative of the $3 \mathrm{D}$ quantum confinement induced by the QD insertions. (Fig. 6e: Reprinted with permission from ref. 76. Copyright (2007) American Chemical Society)

\subsection{3) GaAsSb Nanowires}

As clarified so far in the current review, one of the achievements of growing ternary NWs is the ability to tune the wavelength of the emission by altering the nominal composition of the alloy. The 
case of GaAsSb is of particular interest among other III-V ternary alloys because of the broad coverage of the near-infrared region of the spectrum. The bandgap of GaAsSb can theoretically range from 0.726 $\mathrm{eV}(\mathrm{GaSb})$ to $1.43 \mathrm{eV}(\mathrm{GaAs})$ at room temperature. For that reason, GaAsSb structures have been extensively investigated for applications such as near-infrared photodetectors. ${ }^{[78-79]}$ Besides, this spectrum region is also crucial for telecommunication applications, as GaAsSb emission can reach the O-band (1260 nm-1360 nm) and the C-band (1530 nm-1565 nm). Additionally, GaAsSb has very low hole carrier masses and consequently remarkably high hole mobility among other III-V ternary alloys. These properties are advantageous for the fabrication of high-speed nanoelectronic devices. However, the surfactant effect and poisoning effect of $\mathrm{Sb}$, which will be analysed later on, cause significant alterations at the NWs morphology. The poisoning effect, additionally, reduces the axial growth rate of NWs with increasing Sb content, thus posing a limit to the applicable composition of Sb, below 50\%. ${ }^{[29]}$ Despite this, several groups have devoted to exploring high Sb content in the GaAsSb NWs to enable the emission to reach a larger scale. An additional problem that arises with the incorporation of $\mathrm{Sb}$ in the growing NWs is the trend that $\mathrm{Sb}$ is distributed in an inhomogeneous way in a single NW, thus degrading the functionality of the NW arrays.

There are already reports that describe detailed characterizations and investigation of the optical properties of GaAsSb NWs on Si substrates grown via $\mathrm{CVD}^{[78]}$ and MBE. ${ }^{[27-29,52,79-86]}$ As mentioned earlier, the $\mathrm{Sb}$ content, for structures grown on $\mathrm{Si}$, is kept at a relatively low level. In several cases of growth on patterned Si substrates, where VLS growth occurred, the size of the pattern plays an important role in the morphology and elemental composition of the structures. Decreasing the size of the pitch leads to a higher density of NWs and an enhanced appearance of the shadowing effect. ${ }^{[00,84]}$ Due to the shadowing effect and the strong competition between the NWs, the number of Ga adatoms that incorporated in each of these structures is reduced leading to a radical decrease in the dimensions of the individual NW (Figs. 7a-c). ${ }^{[83]}$ It is worth mentioning that the change in the dimensions of the NWs reduces the wetting angle between the droplet and the NW, a phenomenon which affects the elemental distribution of $\mathrm{Sb}$ in the axis of the NW. ${ }^{[80]}$ Both the height and the diameter of the NWs increase with increasing dimensions of the pitch. The PL spectra also exhibit significant alterations as a result of the changes mentioned earlier, for which the increasing size of the pitch leads to a redshift of the emission peaks (Fig. 7d) ${ }^{[83]}$ The enhanced collection of re-emitted group V flux from the oxide surface and the simultaneous reduced collection of re-emitted group V flux from the NW sidewall facets create compositional variations and lead to the aforementioned redshift of the peaks. ${ }^{[83]}$

There are several interesting effects of the Sb incorporation in the NWs. One of the most interesting intrinsic properties of $\mathrm{Sb}$ is the surfactant effect, due to which, $\mathrm{Sb}$ tends to incorporate at the external facets of the NW, leading to the expansion of the NW diameter with the increasing Sb content. ${ }^{[29,79,83,86]}$ The homogeneous, narrow NWs of Fig. 7e evolve into wide, pillar-like structures (Fig. 7f), upon increasing $\mathrm{Sb}$ content. That trend is also illustrated in the graph of Fig. $7 \mathrm{~g}$. When more $\mathrm{Sb}$ adatoms incorporate in the NW, there is an As-Sb exchange at the surface of the structure, creating a Sb-rich and 
As-deficient environment. The surfactant effect is also related to the poisoning effect, another systematically observed property of Sb. Since Sb atoms occupy spaces in the surface of the NW and owing to the strong bonding between $\mathrm{Ga}$ and $\mathrm{Sb}$, a blockage of the pathway for $\mathrm{Ga}$ adatoms is created, which reduces the amount of Ga that migrates to the droplet and participates in the elongation of the NW. ${ }^{[29]}$ As a direct result, the axial growth rate, hence the length of the NWs is decreased with increasing Sb content (Fig. 7g). Moreover, Sb promotes the crystallization into pure ZB phase, without the presence of obvious polytypes or crystallographic defects. ${ }^{[29,78-79,86]}$ Even at small percentages of $\mathrm{Sb}$, there is a rapid WZ to ZB transition in the crystal. ${ }^{[29,86]}$ In the Bright Field (BF) image of Fig. 7h, a GaAsSb segment is embedded in a GaAs NW. The WZ to ZB transition is noticed at the bottom part of the section where $\mathrm{Sb}$ is incorporated (Fig. 7i). On the contrary, beyond the length of the GaAsSb segment, the crystal structure transits back into WZ (Fig. 7j), showing that the ZB crystal phase of the section is a direct result of the surfactant effect of $\mathrm{Sb}$. It is noticed that both the Sb-induced passivation of surface states and the purity of the ZB phase can lead to a significant improvement of the optical properties of the NWs, via enhancing the intensity of the luminescence. ${ }^{[84]}$ To further improve surface passivation, core/shell structures can be grown, where AlGaAs has been used as shells to neutralize surface states. ${ }^{[28]}$
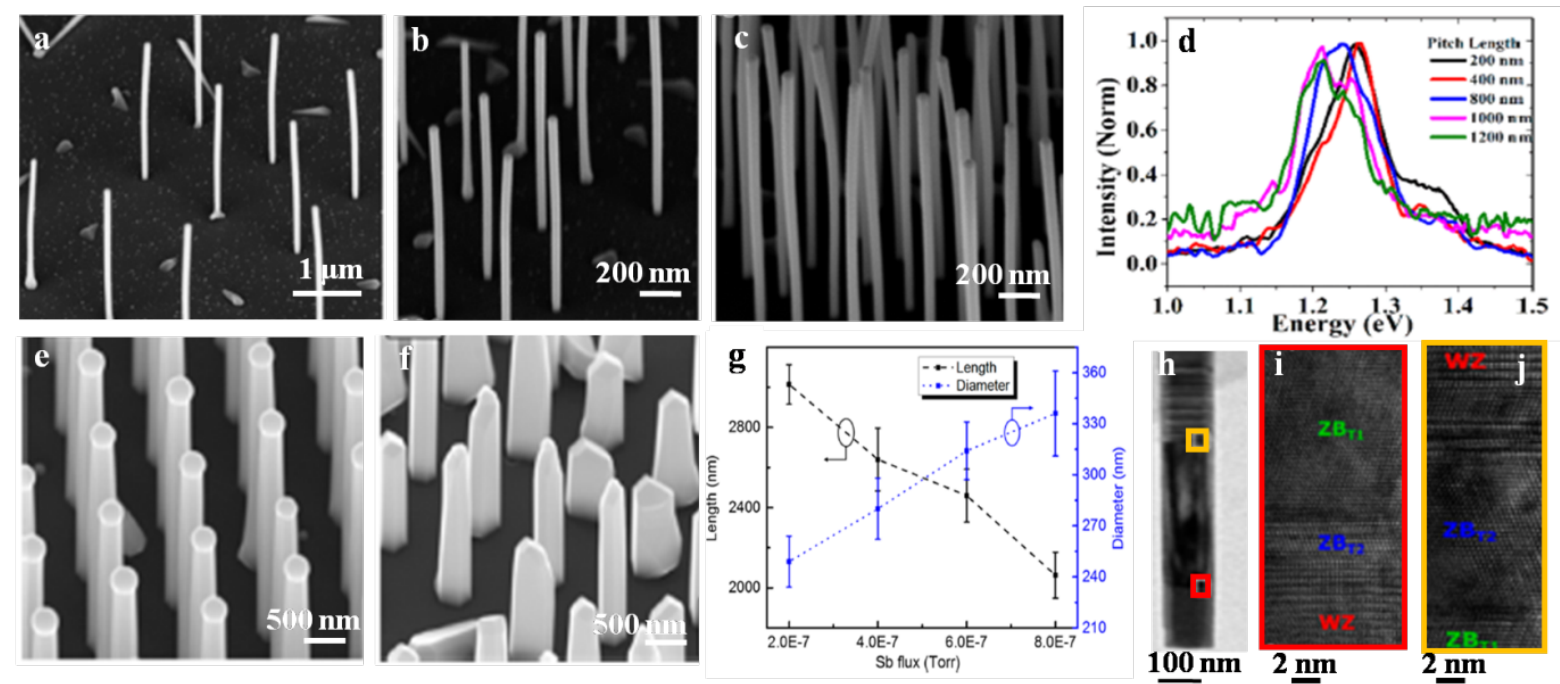

Figure 7: a-c) SEM images of NWs grown on patterned substrate with pitch lengths of 1200, 800 and $400 \mathrm{~nm}$, respectively. The density of the NWs is significantly increased with decreasing length of the pitch d) PL spectra of NWs grown at different pitch lengths. Increasing size of the pitch leads to a redshift of the emission attributed to an increased presence of Sb in the NWs. That is in accordance with the Sb-richness induced by the increasing wetting angle of the droplet with enlarged holes. (Figs. 7a-d: Reprinted with permission from ref. 83. Copyright (2016) American Chemical Society) e-f) SEM images of GaAsSb NW arrays grown under $S b$ flux of $2 \times 10^{-7}$ Torr and $8 \times 10^{-7}$ Torr, respectively. Increasing Sb flux, thus enhanced Sb content, leads to a significant increase in the diameter of the NWs. g) Graph of the diameter and the length of the NWs as a function of the Sb flux. Increasing Sb flux 
causes an increase in the diameter of the NWs as a result of the surfactant effect and a reduction in the length of the structures as a result of the poisoning effect. h) BF-TEM image of a GaAs NW with a GaAsSb segment embedded inside. The red square and the orange square mark the bottom and top part of the segment, respectively. i) High Resolution TEM image of the red square of Fig. 7h. The incorporation of Sb in the NW leads to a rapid WZ to ZB transition. j) High Resolution TEM image of the orange square of Fig. 7h. Beyond the length of the GaAsSb segment, the crystal phase transits back into WZ. (Figs. 7e-j: Reprinted with permission from ref. 29. Copyright (2016) American Chemical Society)

Despite the remarkable features of antimonides, the range of tuning of the elemental composition of GaAsSb NWs grown on Si via MBE has been very narrow. The percentage of Sb does not exceed 50\% for most of the papers mentioned so far, rendering the NWs Sb-poor. ${ }^{[27-28,79,83-85]}$ However, the significance of high wavelength emission for telecommunication applications and infrared light detections leads to concentrated efforts to increase the amount of Sb that is incorporated in the NWs. For instance, an increase in the incorporation of $\mathrm{Sb}$ in GaAsSb NWs grown via MBE was reported by adopting a two-step growth technique, where the temperature was reduced during growth to achieve a higher $\mathrm{Sb}$ incorporation. ${ }^{[52,81-82]}$ In the paper by $\mathrm{Yu}$ et al., ${ }^{[81]}$ apart from the self-catalysed VLS growth that was done in a two-step procedure, they proposed that As background pressure is fixed so that it acts as the As source. That way $\mathrm{Sb}$ content could reach the unprecedented value of $93 \%$. The same technique was adopted in a more in-depth work, which also accomplished GaAsSb NWs with high $\mathrm{Sb}$ composition. ${ }^{[52]}$ This has a profound effect on the optical properties of the NWs. With increasing Sb content by direct increase in Sb flux and one-step growth on Si substrates, the emission is tuned from $844 \mathrm{~nm}$ to $1480 \mathrm{~nm}$ (Fig. 8a). On the other hand, the emission can reach $1760 \mathrm{~nm}$, which is obtained for GaAsSb NWs grown on GaAs stems via the two-step growth method, covering a large range of the near-infrared region of the spectrum (Fig. 8b). ${ }^{[52]}$ 


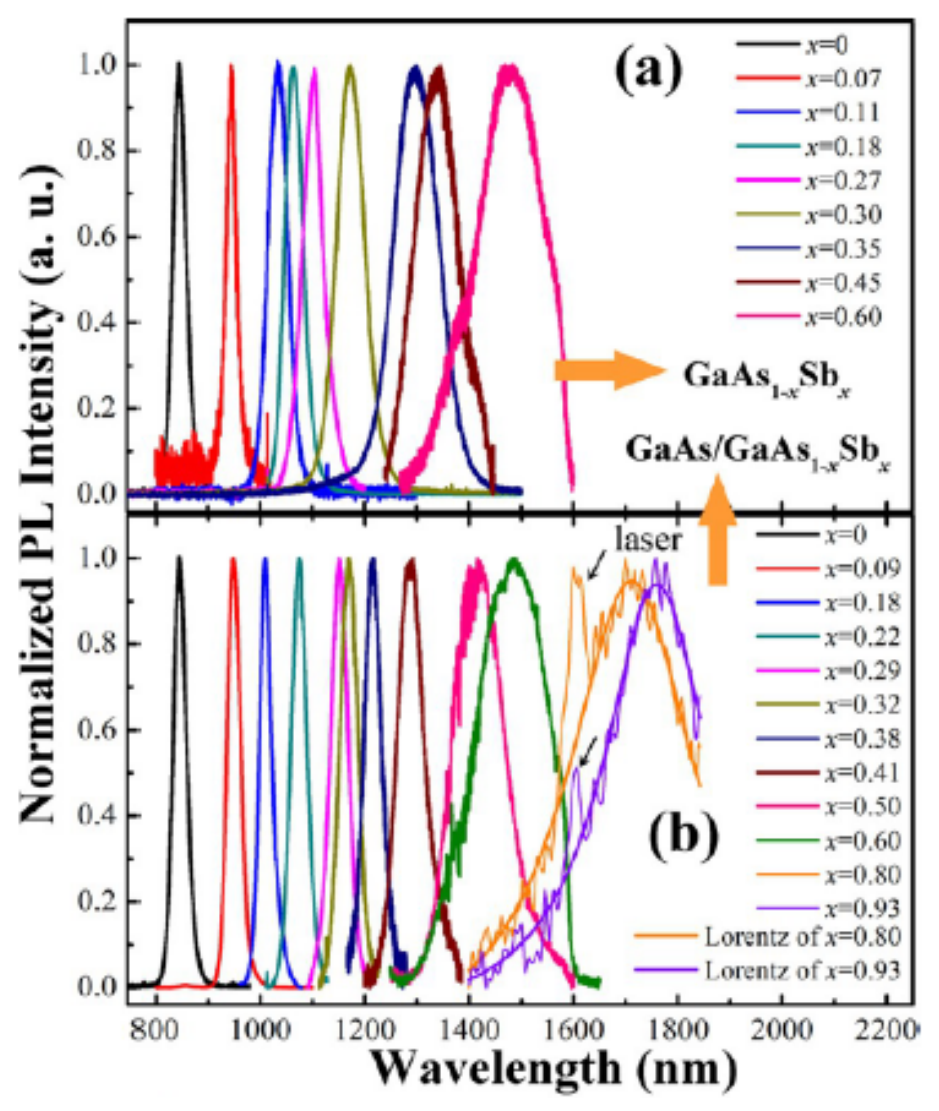

Figure 8: a) Normalized PL spectra of GaAsSb NWs grown on Si substrates, with different Sb content. The measurements are taken at $77 \mathrm{~K}$ and the wavelength of the emission reaches $1480 \mathrm{~nm} . \mathrm{b}$ ) Normalized PL spectra of GaAsSb NWs grown on GaAs stems, with different Sb content. The measurements are taken at $77 \mathrm{~K}$ and the wavelength of the emission reaches $1760 \mathrm{~nm}$. (Figs. 8a-b: Reprinted with permission from ref. 52. Copyright (2017) American Chemical Society)

\subsection{4) InAsSb Nanowires}

After having examined the importance of Sb in ternary III-V NWs by focusing on GaAsSb structures, we will now describe another Sb-related ternary alloy. InAsSb has a bandgap ranging between $0.17 \mathrm{eV}$ (InSb) and $0.36 \mathrm{eV}$ (InAs), making it the ternary alloy with the narrowest bandgap among III-V semiconductors. The energy of the emission can, thus, reach the mid-infrared region of the spectrum and has a great potential for implementation in infrared photodetectors. Furthermore, both InAs and $\mathrm{InSb}$ are semiconductors with remarkably low electron carrier masses and high carrier mobility that renders InAsSb attractive for high-speed FET fabrication. Moreover, InAsSb QWs have been axially embedded in InAs NWs, with a highly bright spontaneous emission up to room temperature, located at the mid-infrared region. ${ }^{[87]}$

The growth of InAsSb NWs on Si substrates has been reported several times, which have been done via MOCVD ${ }^{[88,91-92]}$ and MBE. ${ }^{[30,89-90,93-95]}$ In the majority of the cases, the self-catalysed VLS approach 
was employed, where In droplets were used as catalysts. Similar to GaAsSb NWs, the presence of Sb and its surfactant effect has a significant influence in the morphology and the properties of the NWs. ${ }^{[90]}$ Besides, the low volatility of $\mathrm{Sb}$ also promotes preferential incorporation at the external facets, which explains the quick increase of the diameter. ${ }^{[89,94]}$ As a result, with increasing Sb presence, the diameter largely expands and the length of the NWs is rapidly reduced. ${ }^{[30,88-90]}$ The growth temperature is significant for the morphology and orientation of the NWs are also influenced by the growth temperature. ${ }^{[1]}$ At increased temperatures, the NWs have been vertically aligned on the Si (111) substrate, as observed in the SEM of Fig. 9a. On the contrary, a reduction in temperature from $470^{\circ} \mathrm{C}$ to $450^{\circ} \mathrm{C}$ causes the NWs to change orientation and have a planar configuration on the substrates (Fig. $9 b)$. Growth on other substrate orientations such as (110) and (-100) has also been reported. ${ }^{[92]}$

The crystal structure of InAsSb NWs has also been investigated. The surfactant effect of Sb promotes the formation of pure ZB crystal phase, even when its presence in the NWs is limited. ${ }^{[30,90,94-95]}$ InAs NWs exhibit severe polytypic nature with multiple stacking faults (Fig. 9c). ${ }^{[30,90]}$ The predominant WZ crystal phase is entirely altered upon introduction of $\mathrm{Sb}$ in the material system even in the percentage as low as 3.9\% (Fig. 9d). Upon further increase, the crystal structure is rapidly turned into pure ZB (Fig. 9e) until Sb reaches a maximum of $15 \%$ (Fig. 9f). It is important to mention that because of the catalystfree method used for the growth of the NWs of Figs. 9c-f, the material quality might not be as good as in the case of catalysed NWs. ${ }^{[91]}$ Nevertheless, the ZB percentage was quickly enhanced from an initial value of $19 \%$ to a maximum of $99 \%$, exhibiting the improvement of material quality upon $\mathrm{Sb}$ addition. ${ }^{[30]}$ The improvement of the material quality has direct effects on the electronic properties of the structures. Usually, InAs NW-based photodetectors exhibit a high surface leakage current, due to the intrinsic formation of an electron accumulation layer. On the contrary, InAsSb NW-based photodetectors exhibited a remarkably low leakage current density around $2 \mathrm{~mA} / \mathrm{cm}^{2} .{ }^{[95]}$ The lower noise due to leakage current when compared to other photodetectors is a direct result of the high-quality crystal with increasing $\mathrm{Sb}$ content, even if the structures were grown on a substrate with a high degree of lattice-mismatch. ${ }^{[95]}$

As in any material, the quality of the NWs plays an important role in their optical properties and the existence of polytypism in NW structures causes the formation of a type II band alignment and the subsequent rise of a QW-like peak in the PL spectrum. The premier material quality achieved for InAsSb NWs allowed for the elimination of the type II band alignment, which in turn gave rise to only one peak associated with band-to-band emission. ${ }^{[90]}$ Hence, the purity that was achieved by the incorporation of $\mathrm{Sb}$ in the crystal is significant in improving the optical response of the NWs and is promising for the fabrication of efficient optoelectronic devices. ${ }^{[00,93]}$ In the work by Zhuang et al., for instance, the growth of pure ZB InAsSb NWs and the accomplishment of $19 \% \mathrm{Sb}$ content led to full coverage of the mid-infrared region $(3 \mu \mathrm{m}-5.1 \mu \mathrm{m}) .{ }^{[93]}$ The single crystallinity allowed circumventing the type II band alignment and QW-like peaks, while the shrinkage of the bandgap with increasing Sb 
composition led the emission peak at very low photon energy, approximately $0.23 \mathrm{eV} .{ }^{[93]}$ The corresponding PL spectra at different Sb compositions are presented in Fig. 9g.
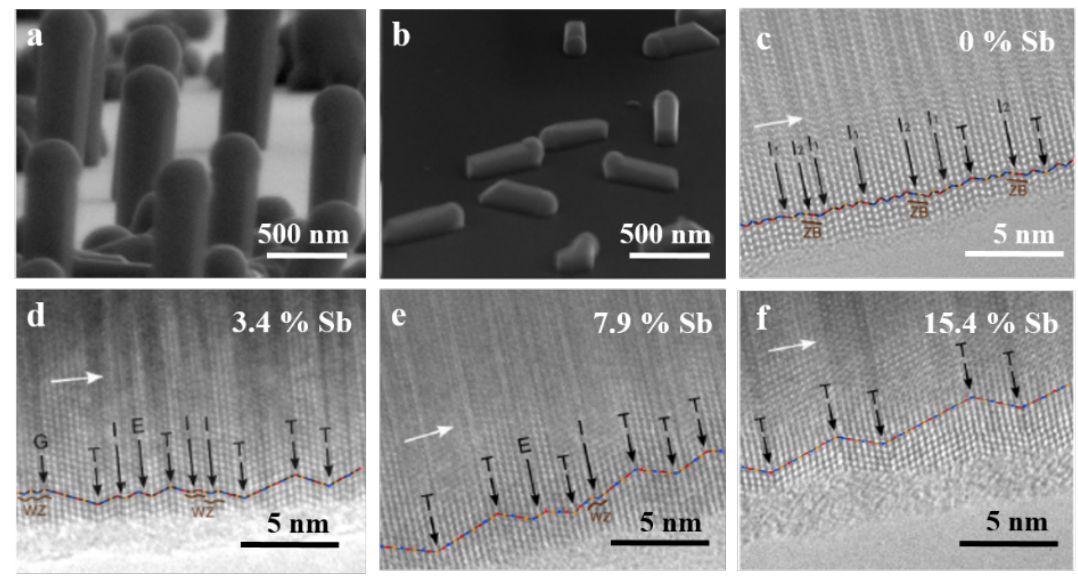

Figure 9: a) SEM image of InAsSb NWs grown at $470^{\circ} \mathrm{C}$. The structures are vertically aligned on the (111)-oriented substrate. b) SEM image of InAsSb NWs grown at $450^{\circ} \mathrm{C}$. The decreasing temperature forces the NWs to change orientation and elongate planar on the substrates. (Figs. 9a-b: Reprinted with permission from ref. 91. Copyright (2015) American Chemical Society) c-f) High Resolution TEM of catalyst free InAs(Sb) NWs grown via the catalyst-free method at different Sb content as indicated in the images. White arrows show the direction of the growth. Increasing Sb content leads to a transition from WZ dominant to pure ZB crystal phase due to the surfactant effect of Sb. (Figs. 9c-f: Reprinted with permission from ref. 30. Copyright (2014) American Chemical Society) g) PL spectra of InAsSb $N W$ s with Sb content of $0,3 \%, 10 \%, 16 \%$ and $19 \%$, respectively. The shrinkage of the bandgap leads to peaks covering the entire mid-infrared region, reaching $0.23 \mathrm{eV}$. No QW-like peaks are noticed, due to the single crystallinity of the NWs. (Fig. 9g: Reprinted with permission from ref. 93. Copyright (2017) IOP Publishing Ltd.)

\section{2) III-III-V Nanowires}

\subsection{1) AlGaAs Nanowires}

Another important ternary alloy is AlGaAs, which has great potential in the realization of lasers and other optical applications. AlGaAs has a bandgap theoretically ranging between $1.43 \mathrm{eV}$ (GaAs) and $2.12 \mathrm{eV}$ (AlAs) at room temperature. Experimentally, the growth of AlAs NWs on Si has not yet been reported and the nominal composition of $\mathrm{Al}$ in $\mathrm{AlGaAs}$ alloys cannot cover the entire range between the two mentioned values, so far. From the literature, the highest value of $\mathrm{Al}$ that has been reported in AlGaAs NWs on $\mathrm{Si}$ is $60 \%$ and further efforts for increasing the $\mathrm{Al}$ content has led to inhomogeneous, 
tapered or conical NWs with a highly irregular morphology. ${ }^{[96]}$ The lattice matching of AlGaAs and GaAs makes this ternary alloy particularly attractive for the realization of GaAs-based near-infrared devices in the form of core/shell NWs, where AlGaAs shell surrounds the GaAs core. Indeed GaAs/AlGaAs core/shell heterostructures have been frequently employed for optical applications, where the shell protects the core from oxidation, confines the carriers in the GaAs core region and passivates surface states, ${ }^{[97]}$ and these straightforwardly lead GaAs/AlGaAs core/shell NWs to exhibit good optical properties with enhanced PL emission and strong polarization. ${ }^{[98-99]}$ Additionally, they are employed for laser fabrication, with good features up to room temperature. ${ }^{[100-101]}$ The shape-driven segregation of $\mathrm{Al}$ at the vertices of the hexagonal shell has also led to the generation of self-assembled AlGaAs QDs embedded in the shell of GaAs/AlGaAs NWs, a structure which exhibits optimum emission properties. ${ }^{[102]}$

On the other hand, the work on axial AlGaAs NWs is more limited. Available relevant reports on AlGaAs NWs on GaAs (111)B substrates highlighted the optimum optical properties of this ternary alloy. ${ }^{[103-104]}$ Literature on Si substrates may be less but has some significant observations as well which will be shown in the next paragraph. The growth of AlGaAs NWs has been achieved via MBE ${ }^{[66,105]}$ and $\mathrm{MOCVD}^{[106]}$ following the VLS growth mode. In all of the up-to-date reports, the procedure was $\mathrm{Au}$-assisted. The presence of $\mathrm{Au}$ introduces possible contamination in the system, as stated in the previous section.

The growth parameters play a crucial role in the case of AlGaAs NWs. The duration of the growth is of primary importance. A narrow time frame does not allow for NWs to be developed and adopt their axial morphology, while a wide time frame allows for the incorporation of more semiconductor adatoms and causes the elongation of the NWs. ${ }^{[96]}$ It is also noted that higher percentages of Al lead to different morphology of the NWs. Increasing Al content from $10 \%$ to $30 \%$ forces the NWs to adopt a pencil like morphology (Fig. 10a). ${ }^{[96,105]}$ Further increase leads to the formation of conical structures with a pronounced tapering at their tips. The diameter of the NW tip is defined by the diameter of the droplet. On the other hand, the spontaneous formation of an AlGaAs shell due to the shorter diffusion length of $\mathrm{Al}$ is also exhibited. These two factors cause a tapered shape in the NW, with a large base and a narrow tip. ${ }^{[96]}$ The Al-rich shell has been reported by a few reports on AlGaAs NWs and is attributed to the low diffusion length of $\mathrm{Al}$, which tends to incorporate at the external facets of the NW, forming a shell-like feature. ${ }^{[96,105-106]}$ Spontaneous shell growth is not uncommon in ternary NWs and will be mentioned again for other ternary alloys. The presence of the shell is revealed in cross-sectional EDX measurements, as Al peaks and corresponding Ga dips at the outer parts of the NW (Fig. 10b). ${ }^{[105]}$ Furthermore, the Al-rich shell affects TEM images, since the reduced presence of the heavier element, which in this case is Ga, leads to a darker contrast in the external facets of the NW (Fig. 10c). ${ }^{[105]}$ It is noted that the $\mathrm{Au}$ droplet is rapidly emptied of $\mathrm{Al}$ components while it has a small presence of As, owing to its low solubility in $\mathrm{Au}$, that barely reaches $1-2 \%$. On the contrary, the presence of $\mathrm{Ga}$ in the droplet is much more enhanced. ${ }^{[105]}$ Besides, the temperature also influences the morphology of the 
structures, as increasing temperature slows down the axial growth rate due to reduction in the level of supersaturation in the Au droplet. ${ }^{[96]}$ In the SEM image of Fig. 10a, the NWs depicted are grown at $510^{\circ} \mathrm{C}$ and have a good morphology, for which the $\mathrm{Al}$ content is approximately $30 \%{ }^{[105]}$ It is noted that in some cases the unintentional formation of a 2D AlGaAs layer on the Si substrates may occur. Nevertheless, this layer is optically inactive and does not participate in the PL emission. ${ }^{[96]}$
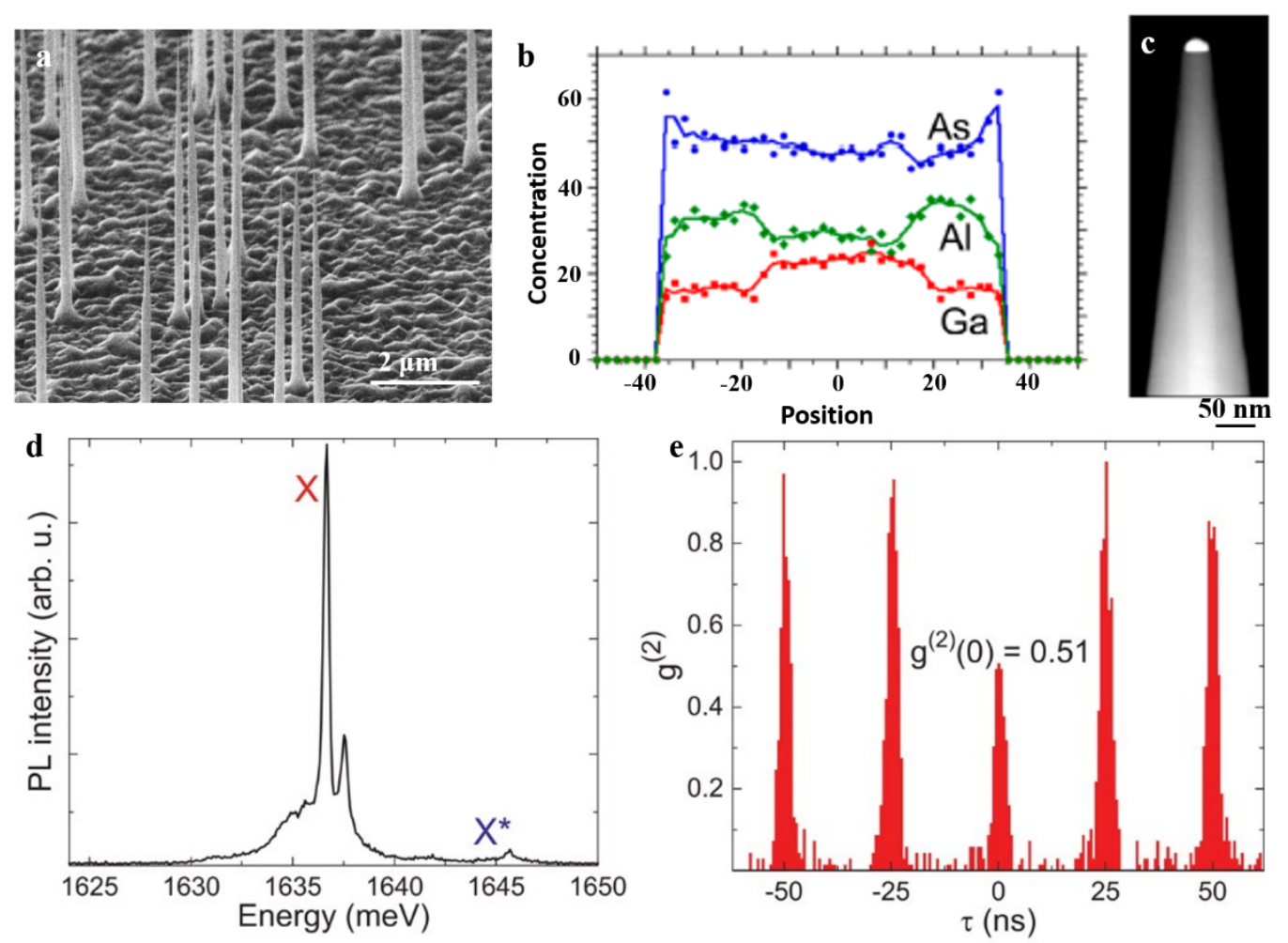

Figure 10: a) SEM of an AlGaAs NW array grown on Si at $510^{\circ} \mathrm{C}$ with a nominal composition of Al at 30\%. b) Cross sectional EDX scanning of an AlGaAs NW. The peaks of Al and dips of Ga at the external facets reveal the presence of an Al-rich shell. As expected, Arsenic is homogeneously distributed in the structure. c) TEM image of a $N W$, revealing its inversed tapered shape. The darker colour of the shell reveals its higher percentage of Al. (Figs. 10a-c: Reprinted with permission from ref. 105. Copyright (2016) American Chemical Society) d) PL spectrum of GaAs QDs embedded in GaAs/AlGaAs core/shell NWs. Two peaks are exhibited, $X$ and $X^{*}$ attributed to exciton and biexciton transition, respectively. The linewidths for $X$ and $X^{*}$ are $315 \mu \mathrm{eV}$ and $458 \mu \mathrm{eV}$. e) Second order correlation function of $X$, revealing antibunching behaviour and demonstrating the potential of the structure to act as a non-classical light source. (Figs. 10d-e: Reprinted with permission from ref. 107. Copyright (2015) American Chemical Society)

The importance of NWQDs was already described earlier for GaAsP NWs. ${ }^{[71]} \mathrm{AlGaAs}$ NWs are ideal for hosting GaAs QDs, due to the lattice matching of the two alloys, causing negligible, if any, amount 
of strain. The presence of the QD in the NW results in an additional emission peak attributed to the QD segment. ${ }^{[96]}$ When NWs are grown at higher temperatures, the intensity of the signals is significantly reduced, and the difference between the QD peak and the NW peak becomes negligible. ${ }^{[96]}$ Additionally, $\mathrm{Al}$ content influences the emission properties of the structures, as increasing $\mathrm{Al}$ leads to a blueshift of the NW emission. It is noted, however, that the QD emission can be tuned by altering the size of the dot and as a result, the corresponding peak can be maintained at precisely the same wavelength, for different elemental composition of the NW. ${ }^{[96]}$ Ultranarrow emission peaks have been observed, deriving from GaAs QDs in GaAs/AlGaAs core/shell NWs (Fig. 10d). ${ }^{[107]}$ The low-energy peak, attributed to exciton transition exhibits a linewidth of $315 \mu \mathrm{eV}$, while the high-energy peak is attributed to biexciton transition and demonstrated a linewidth of $458 \mu \mathrm{eV}$. These results are comparable with the best ones obtained from the commonly grown Stranski-Krastanov QDs (SKQDs). ${ }^{[108]}$ Furthermore, these NWQDs act as non-classical light sources and exhibit photon antibunching behaviour, with a second order correlation function at zero time delay being 51\% (Fig. 10e). ${ }^{[107]}$ Moreover, axial heterostructures may include consecutive segments of GaAs and AlGaAs respectively, where AlGaAs insertions act as quantum wells (QWs) ${ }^{[106]}$ which shows the crystal features of such NWs depends on the growth temperature.

\subsection{2) InGaAs Nanowires}

The bandgap of InGaAs covers the entire range from the near-infrared to mid-infrared, with values between $0.36 \mathrm{eV}$ (InAs) and $1.43 \mathrm{eV}(\mathrm{GaAs})$ at room temperature. InGaAs NWs have been used for solar cell fabrication with very good results. ${ }^{[20]}$ Additionally, they have been implemented for the fabrication of FETs, by following a surround-gated morphology, with optimum performance in electron transport owing to its high electron mobility. ${ }^{[31]}$ Optically pumped laser device based on InGaAs NWs has also been reported, ${ }^{[109]}$ while embedding InGaAs QDs in GaAs NWs has been proven beneficial for near-infrared operating lasers. ${ }^{[110]}$

The growth of InGaAs NWs has been achieved via MOCVD,${ }^{[31,43,50,111-114]} \mathrm{CVD}^{[32]}$ and MBE, ${ }^{[20,109,115-}$ ${ }^{116]}$ either via a catalyst free-approach or by using $\mathrm{Au}, \mathrm{Ga}$ or In droplets as catalysts. The effect of different parameters on the morphology of the NWs has been examined intensely. The growth temperature has a significant impact on the morphology of the NWs by promoting VS lateral growth and forming a shell. This may induce tapering and degrade the morphology of the structures, while it can also affect the elemental distribution of In as it favours In incorporation at the external shell. ${ }^{[12]}$ In the work by Hou et al, ${ }^{[32]}$ upon reducing the temperature from annealing to growth, severe kinking of the structures occur, which were solved by using a two-step method to grow InGaAs NWs in the same paper. By doing so, a high temperature of the nucleation stage eliminated any solid phase residues within the droplet, while the reduction in temperature during growth ensures the verticality of the NWs, which led to straight, unkinked NWs with high uniformity and single crystallinity. ${ }^{[32]}$ Besides, V/III 
ratio is also crucial for the growth, as increasing group V flux reduces the migration of group III adatoms in the catalyst droplet and further enhances lateral expansion of the NWs. ${ }^{[12]}$

Similar to AlGaAs NWs, the growth of III-III-V ternary NWs is more challenging compared with III-V-V ternary NWs since the catalyst droplet is a group III element, which acts as both the collector and supplier, and altering group III fluxes while maintaining the stable growth is difficult due to the replacement of the component that consist of droplet. Fortunately, both Ga and In can act as catalyst droplets. Taking advantage of this fact and by adopting the apparently different growth window for NWs growth with In and Ga droplets respectively, a full coverage of the compositional range can be achieved. It has been shown that in order to obtain In-rich, InGaAs NWs the ideal temperature would be $550^{\circ} \mathrm{C}$, while the temperature for Ga-rich NWs is $610^{\circ} \mathrm{C} .{ }^{[115]}$ This is probably attributed to the lower mobility of Ga adatoms, which requires for a higher temperature in order for Ga incorporation in the NWs to be promoted. The above are illustrated in the SEM images of Figs. 11a-h, where InGaAs NWs were grown at different temperatures and adopted different Ga composition. ${ }^{[115]}$ The NWs of this work covered the entire compositional range between InAs and GaAs, even though two growth regimes were employed for In-rich and Ga-rich structures, respectively. The crystal structure is also altered at different Ga content. In-rich structures are crystallized into the WZ phase, with the appearance of several stacking faults, while Ga-rich NWs exhibit ZB structure with occasional rotational twins. ${ }^{[15]}$ The optical properties of NWs can also be widely tuned, as the energy of the emission can range between $0.42 \mathrm{eV}$ and $1.1 \mathrm{eV}$ with increasing Ga composition (Fig. 11i).

In a wafer scale, the distribution of NWs is important for the potential device fabrication. For the MOCVD growth, the density of InGaAs NWs demonstrates slight variations, as presented in the optical image of Fig. $11 \mathrm{j} .{ }^{[114]}$ The rainbow-like feature in the image depicts the differences in the NW density, with less NWs being formed at the edges of the substrate. The morphology of the structures is also affected by In composition. Decreasing In content leads to NWs with a larger diameter, averagely. ${ }^{[14]}$ This increase in the diameter can be viewed from the SEM images of Figs. $11 \mathrm{k}$ and 111 , with $85 \%$ and $30 \%$ In content, respectively. ${ }^{[114]}$ It is also noted that for each of these cases, the temperature was optimized. For $85 \%$ In content, the growth temperature was $570^{\circ} \mathrm{C}$ (Fig. 11k) while for decreasing In content, the optimized temperature increased at $590^{\circ} \mathrm{C}$ (Fig. 111). 

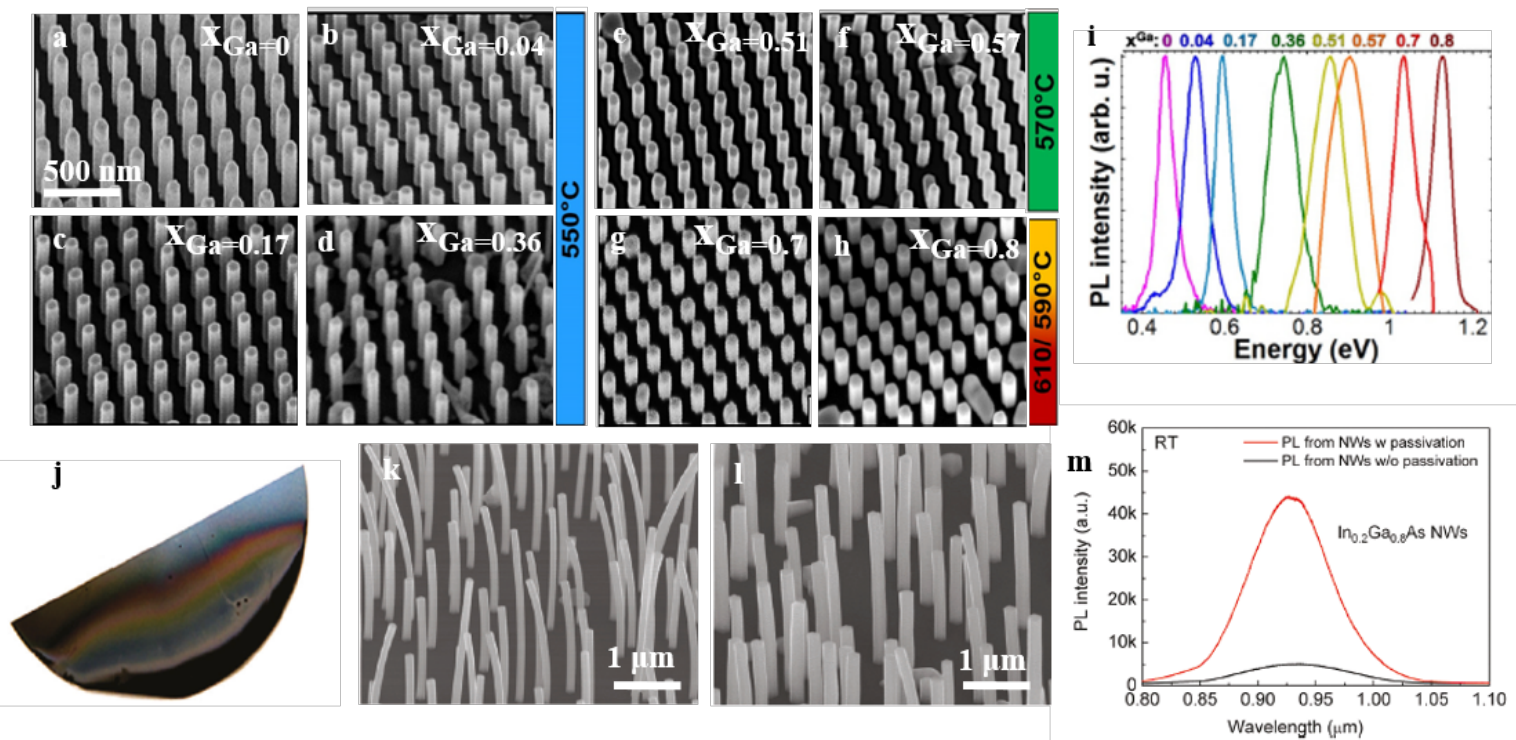

Figure 11: a-h) SEM images of InGaAs NWs grown via MBE following SAG mode. The arrays of the grown NWs have a high level of homogeneity. NWs were grown at different temperatures with different Ga compositions, as indicated in each image i) Normalized micro PL spectra of InGaAs NWs grown at different Ga composition. A wide range of tuning is accomplished. (Figs. 11a-i: Reprinted from ref. 115 with the permission of AIP publishing.) j) Optical image of a Si (111) wafer with InGaAs NWs grown. The slight variations in the $N W$ density are demonstrated by the colour variations in the image. k-l) SEM images for InGaAs NWs with $15 \%$ and $70 \%$ In content grown at $570^{\circ} \mathrm{C}$ and $590^{\circ} \mathrm{C}$, respectively. Increased composition of In causes an increase in the diameter of the NWs, due to its incorporation at the external facets. m) Micro-PL spectrum of an InGaAs NW array, with 20\% In depicted as a black line. The red line corresponds to a similar array with the addition of a GaAs shell. The passivation of surface states by the shell leads to a considerable increase of the PL intensity. (Figs. 11j-m: Reprinted with permission from ref. 114. Copyright (2011) American Chemical Society)

The passivation shell was highlighted in the optical properties of the same InGaAs NWs and it was found that the passivation of surface states enhances the PL intensity significantly. ${ }^{[14]}$ In the micro-PL spectrum of an InGaAs NW array in Fig. 11m, a clear peak is observed originating from InGaAs NWs, located at $0.92 \mathrm{eV}$ (black line). The red line of the spectrum depicts core/shell NWs, where the GaAs shell cladded the InGaAs core while the black one is for those without GaAs passivation shell. As in this case, for which the formation of a shell cladding the NW core can passivate surface states and ameliorate the optical performance of the NWs, the growth of an InAlAs shell surrounding the InGaAs core has been proven beneficial in the applicability of NWs in solar cells. ${ }^{[20]}$ With the Ga content in the core and the $\mathrm{Al}$ content in the shell kept at a stable level of $47 \%$, the alloys are lattice matched and no strain is introduced in the NW by that mechanism. Another novel morphology was suggested by Tomioka et al., who created a core/multishell structure in order to improve the performance of InGaAs NWs devices. ${ }^{[31]}$ The complex structures are composed of an InGaAs core, followed by an InP barrier 
layer and an InAlAs shell that included a $\delta$-doped region. Finally, the entire structure is cladded by an InGaAs outer shell.It is noted that this configuration enabled the fabrication of efficient, surround-gated FETs.

\subsection{3) InGaP Nanowires}

Another important ternary alloy for optical and energy applications is InGaP, which has a bandgap ranging between $1.344 \mathrm{eV}(\mathrm{InP})$ and $2.24 \mathrm{eV}(\mathrm{GaP})$ at room temperature. As mentioned earlier, $\mathrm{GaP}$ has an indirect bandgap. However, an indirect-to-direct transition can occur when the alloy is crystallized into the $\mathrm{WZ}$ phase for GaP. This has not been realized yet on Si but is very promising for achieving green light emission and covering the entire compositional range between direct bandgap $\mathrm{GaP}$ and $\mathrm{InP}$, when integrated with the Si platform. In combination with quaternary AlGaInP, InGaP NWs on GaP substrates have been demonstrated for the realization of orange, orange-red, yellow, and green LEDs. ${ }^{[117-118]}$ They can also be implemented in the fabrication of multijunction solar cells, with high efficiency. ${ }^{[119]}$ Despite that the elastic strain relaxation that NW geometry offers is an extremely advantageous characteristic, allowing substrate insensitivity in the growing NWs, the growth of InGaP NWs on Si is far from being fully understood, and the available work on this ternary alloy is minimal. Up to our knowledge, there is only one report focusing on the monolithic growth of these ternary NWs on $\mathrm{Si}$ which is based on a composition gradient achieved via a particular technique they employed, where they performed a horizontal tilting of the substrate to achieve a temperature gradient. ${ }^{[120]}$ Ternary $\mathrm{InGaP}$ has also been grown as a double shell, surrounding a GaP NW core on Si substrates, achieving visible light emission. ${ }^{[121]}$

On the contrary, the literature on $\mathrm{InGaP}$ NWs grown on substrates other than $\mathrm{Si}$ is more abundant. InGaP NWs have been grown via $\mathrm{MBE}^{[122]}$ or $\mathrm{MOCVD}^{[123-125]}$ on various semiconductor substrates. The elemental composition of the alloy has covered the entire range from GaP to InP. ${ }^{[122]}$ Their morphology is mostly dependent on the growth parameters as in the case of previously described ternary NWs. For example, decreasing group III impingement rate leads to a gradual tapering of the structures. On the other hand, increasing V/III ratio promotes lateral VS growth, leading to a pencil-like shape of the NWs. Another finding regarding the morphological features of InGaP NWs is the spontaneous formation of a Ga-rich InGaP shell, as previously mentioned for other ternary alloys, which is attributed to the lower diffusion length of Ga when compared to In. The promotion of lateral VS growth via the incorporation of Ga adatoms at the external facets of the NWs can hinder their axial elongation and can be detrimental to the optical properties of the structures. A suggested way to eliminate or limit this phenomenon is $\mathrm{HCl}$ etching in-situ during the growth procedure. ${ }^{[123]}$ That method also significantly reduces the volume of the InGaP NWs. Another essential factor in determining the morphology of the NWs is the growth temperature. ${ }^{[124]}$ At low growth temperatures $\left(625^{\circ} \mathrm{C}-650^{\circ} \mathrm{C}\right)$, the length of the structures is shorter while radial overgrowth is promoted (Fig. 12a). The competition between neighbouring NWs causes 
severe abnormalities in the morphology of the structures. Between $675^{\circ} \mathrm{C}$ and $750^{\circ} \mathrm{C}$, NWs are highly homogeneous in dimensions, vertically aligned on the substrate, forming uniform arrays (Figs. 12b-d). At higher temperatures, the axial growth rate was further promoted, resulting in a more pronounced suppression of the radial growth. However, severe bending occurred, probably stemming from the strain that is induced in the structures by a slight compositional inhomogeneity at these high temperatures (Figs. 12e-f).Ga composition is a factor that plays a crucial role in the optical properties of the NWs. In Fig. 12g, cathodoluminescence (CL) spectra of samples with different Ga composition are depicted. It is obvious that with increasing $\mathrm{X}_{\mathrm{TMGa}} /\left(\mathrm{X}_{\mathrm{TMGa}}+\mathrm{X}_{\mathrm{TMIn}}\right)$, which led to an increase in Ga content, the emission peaks are shifted towards shorter wavelengths. It is noted that increasing Ga content also led to a morphological change of the structures, with polycrystals being formed instead of standing NWs. ${ }^{[124]}$

Besides, the crystal structure of InGaP NWs has also been investigated. The NWs grown via MBE are crystallized mainly in the WZ phase, regardless of the growth parameters. However, several ZB monolayers in the form of stacking faults are exhibited periodically, within the predominant WZ segments. ${ }^{[122]}$ The density of the stacking faults is decreased with decreasing group III impingement rate or with increasing V/III ratio and the NW crystal approaches purity in the WZ phase. The opposite trend was observed for NWs grown via MOCVD, with in-situ $\mathrm{HCl}$ etching. ${ }^{[123]}$ The predominant crystal phase, in this case, was $\mathrm{ZB}$, with occasional twins being formed but no WZ signal deriving from the InGaP part of the structure. ${ }^{[123]}$ In ref. 125 , the introduction of diethylzinc in the MOCVD reactor during SAG of NWs leads to a promotion of ZB crystal phase. It is interesting to observe that increasing diethylzinc concentrations leads to a rapid reduction of the In content in the NW, as depicted in the corresponding graph (Fig. 12h), which is also supported by the Ga peaks in the axial Energy Dispersive X-Ray Spectroscopy (EDX) scanning of the NW, confirming Ga-richer p-doped regions and In-richer intrinsic regions along with the structure (Fig. 12i). This higher incorporation rate of Ga is suggested to stem from the increased efficiency in the pyrolysis of TMGa in the presence of diethylzinc, which in turn results in a higher Ga supply in the growth front. 

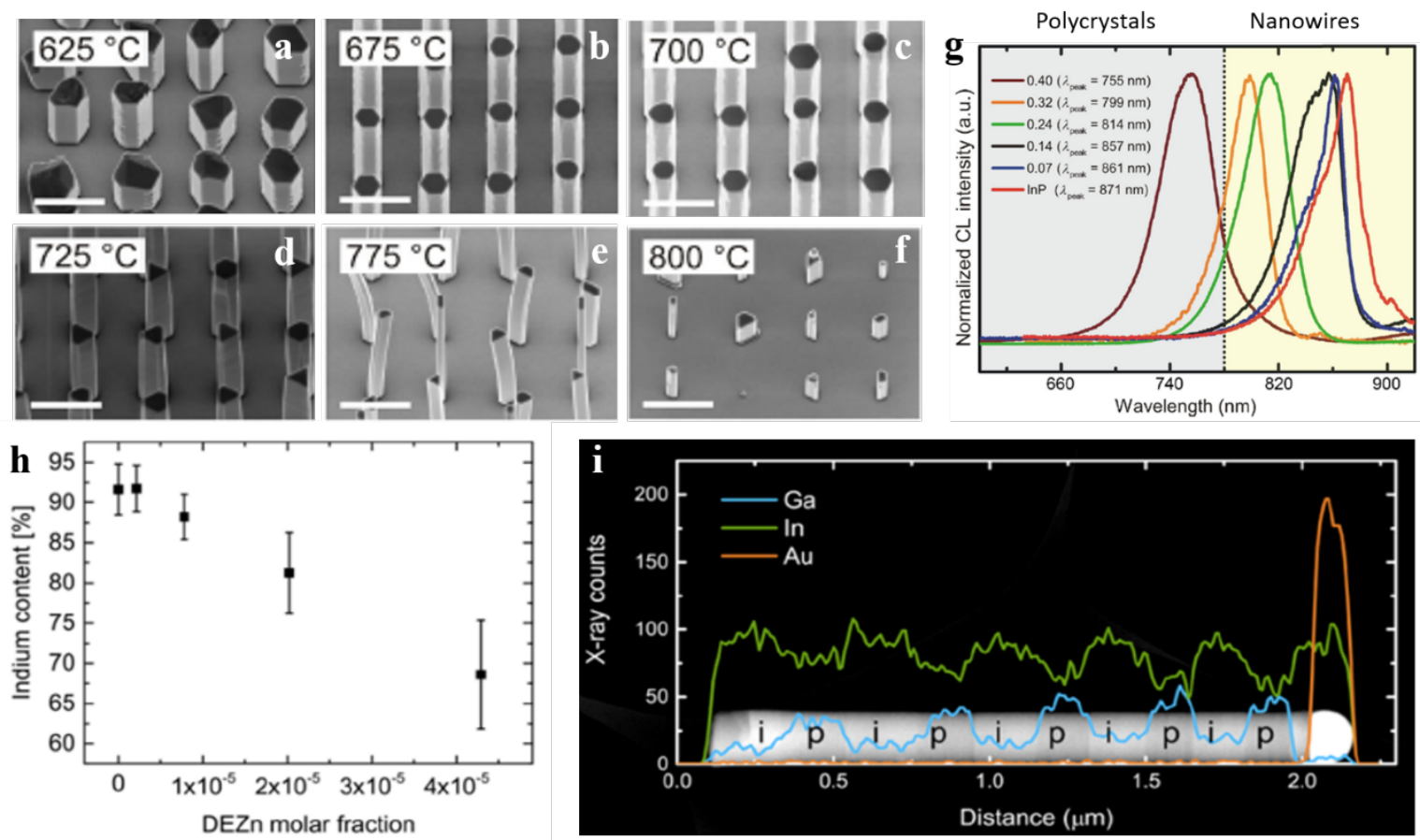

Figure 12: a-f) SEM images of InGaP NWs grown on InP substrates. The different temperatures of the growth are noted in the images. It is observed that low growth temperatures led to shorter NWs and promoted radial overgrowth (Fig. 12a), while increasing temperatures induced the formation of highly homogeneous arrays of perpendicular NWs (Figs. 12b-d). Further increase above $750^{\circ} \mathrm{C}$ in temperature led to compositional inhomogeneity that induced strain in the structures and ultimately caused their bending and non-vertical orientation (Figs. 12e-f). g) CL spectra of InGaP NWs grown with different Ga composition. A blueshift related to the increased Ga content is presented with increasing flow ratio. After a critical point, polyrcrystals were formed instead of NWs. The shortest wavelength for standing NWs in this work was $799 \mathrm{~nm}$, while the highest was obtained for InP NWs at $871 \mathrm{~nm}$. (Figs. 12a-g: Reprinted by permission from Springer Nature: Nano Research, ref. 124. Copyright (2017) i) Graph of the In content as a function of the diethylzinc molar fraction. It is clear that increasing presence of diethylzinc leads to a rapid reduction in the In composition of the structure. j) EDS line scanning of an InGaP NW with consequent $i$ - and p-regions. Ga peaks at the doped regions reveal increased presence of this element and confirm that the presence of diethylzinc causes a reduction in the In content and a subsequent increase in the Ga content. (Figs. 12h-i: Reprinted with permission from ref. 125. Copyright (2017) American Chemical Society) 


\section{3) InGaSb, InPSb, GaPSb and AlInP}

After having examined some of the most heavily investigated ternary alloys NWs, we will focus on four other alloys that have not been explored much, for which the growth on $\mathrm{Si}$ is particularly challenging. It is noted that the growth of NWs based on three of these ternary alloys has not yet been reported on Si substrates, but their necessity renders their growth on other substrates, as well as $\mathrm{Si}$, a great accomplishment. For that reason, we find it necessary to briefly describe each of them, which are III-III-V InGaSb and InAlP and III-V-V GaPSb and InPSb.

$\mathrm{InGaSb}$ is an alloy with great potential especially for high speed electronics and CMOS technology applications, as one of its edge alloys, InSb, has the highest electron mobility among III-V semiconductors and the other edge alloy, GaSb, has the highest hole mobility. This intriguing feature leads to InGaSb being an ideal candidate both for electron-based and hole-based electronic devices, depending on its composition. Its bandgap ranges from $0.17 \mathrm{eV}(\mathrm{InSb})$ to $0.726 \mathrm{eV}(\mathrm{GaSb})$ at room temperature. This coverage allows for the implementation of InGaSb NWs in infrared photodetectors and thermovoltaic devices, along with high speed electronics. Up-to-date, we are only aware of two reports on the growth of InGaSb NWs on InAs (111)B substrates via MOCVD. ${ }^{[45,126]}$ In these reports, Ghalamestani et al. used a wire-on-stem growth technique, which enabled them to grow InGaSb NWs successfully. This was done by adopting the wire-on-stem technique, where first InAs stems were grown, followed by InSb stems and then InGaSb segments (Fig. 13a). The NW segments exhibit differences in the diameter, the first one being attributed to the $\mathrm{WZ}$ to $\mathrm{ZB}$ transition after incorporation of $\mathrm{Sb}$ in InAs/InSb interface and the second one being attributed to the higher growth rate of InGaSb when compared to InSb (Fig. 13b). ${ }^{[126]}$ The NWs that were grown had a Ga content of $60 \%$ (Fig. 13c).

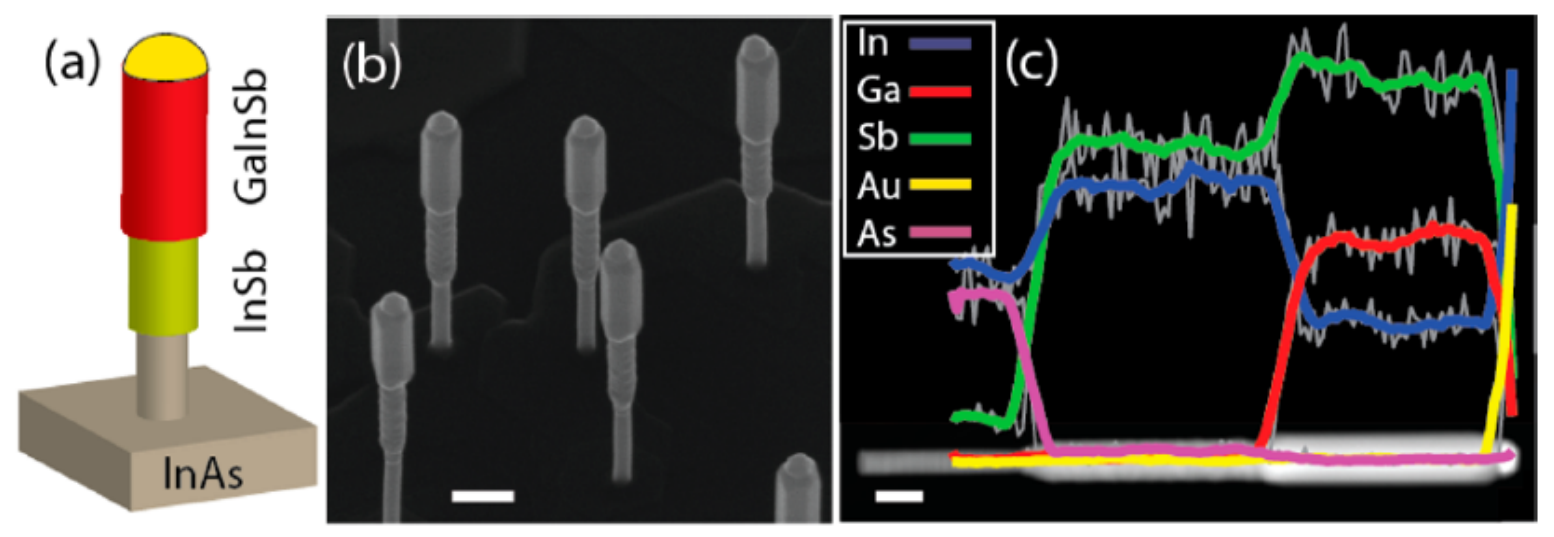

Figure 13: a) Schematics of the InGaSb NW, showing the three different segments (InAs stem, InSb stem, InGaSb segment) of the structure. b) SEM image of the wire-on-stem structures, clearly exhibiting differences in the diameter, between the consecutive segments. The scale bar is $200 \mathrm{~nm}$ c) EDX axial scanning of the NW. The InGaSb regions correspond with the increase in the Ga line (red). This segment 
is revealed to be Ga-rich, with approximately $60 \%$ Ga content. The scale bar is $100 \mathrm{~nm}$. (Figs. 13a-c: Reprinted with permission from ref. 126. Copyright (2012) American Chemical Society)

On the other hand, InPSb is a ternary alloy with a highly tuneable bandgap between $0.17 \mathrm{eV}(\mathrm{InSb})$ and $1.344 \mathrm{eV}(\mathrm{InP})$ at room temperature. As a result, it is particularly attractive for electronics, solar cells and most importantly mid-infrared photodetectors. Nevertheless, the large lattice mismatch between InP and InSb (approximately 10.4\%) and lack of a suitable substrate renders its thin film growth challenging, for which NW geometry may be feasible, as it allows for the combination of materials with high lattice mismatch by elastic relaxation of strain. The difficulties in the growth of InPSb led to just one report on their development and characterization. The InPSb NWs were grown via MOCVD on InP (111)B substrates ${ }^{[127]}$ and exhibit a highly tuneable morphology, crystal phase and elemental distribution, depending on the growth temperature. A temperature gradient that can be formed on the substrate leads to central NWs adopting a fully formed, 1D NW morphology, while moving towards the edge, NWs are formed initially as cones and ultimately as short nanopillars. ${ }^{[127]}$ This difference is connected to the low, intermediate and high temperature while moving from the centre of the substrate to its edge.

One of the most important fields where NWs can play an active role is energy harvesting, in solar cells and water splitting devices. Especially GaP exhibits stability as photocathode for water splitting devices and ideal band alignment for $\mathrm{CO}_{2}$ reduction. Its bandgap is indirect and has a value of $2.3 \mathrm{eV}$ at room temperature. Alloying $\mathrm{GaP}$ with $\mathrm{GaSb}$ enables a wide tuning of the bandgap energy from 0.726 $\mathrm{eV}(\mathrm{GaSb})$ and $2.24 \mathrm{eV}(\mathrm{GaP})$ and contributes in an indirect to direct transition, which is beneficial for solar cells and optoelectronic applications but also unbiased water splitting reaction. ${ }^{[128]}$ The alloying of $\mathrm{GaP}$ with $\mathrm{GaSb}$ introduces Sb-related impurity states the edges of the bandgap, which lead to the lowering of the bands at the $\Gamma$-point and, in turn, cause an indirect to direct transition of the bandgap. ${ }^{[128]}$ Despite these features, up to our knowledge there is only one report on the growth and investigation of GaPSb NWs grown via reactive vapour transport in a microwave plasma reactor by adopting a selfcatalysed approach on (111) Si substrates. In micro-photoluminescence (PL) measurements of GaPSb NWs in ref. 128, the spectrum deriving from the NWs shows two clear peaks located at $1.73 \mathrm{eV}$ and $1.82 \mathrm{eV}$ at $77 \mathrm{~K}$. It is noted that measurements on $\mathrm{GaP}$ reference sample reveal a single peak at $2.3 \mathrm{eV}$, corresponding to the bandgap of $\mathrm{GaP}$, which is entirely suppressed at room temperature, owing to its indirect bandgap nature. The spectrum at room temperature shows multiple peaks corresponding to the values of the direct bandgaps exhibited in NWs with different elemental composition which range between $1.73 \mathrm{eV}$ and $2.21 \mathrm{eV} \cdot{ }^{[128]}$ It is interesting to notice that the excellent optical properties and the indirect to direct transition in GaPSb NWs are observed even at very low Sb presence, down to $\%$.

As mentioned throughout the current review, NWs are promising as building blocks for the fabrication of important optical devices such as lasers and LEDs. Solid state lighting via LEDs is particularly attractive because of their bright emission, high efficiency, and long lifetimes. However, a 
significant obstacle that is encountered is the phenomenon known as the 'green gap', where the efficiency of the devices in the yellow and green regions is very low. AlInP NWs have a direct bandgap in the green region and are, thus, ideal candidates for the realization of green-emitting LEDs. Their bandgap theoretically ranges between $1.344 \mathrm{eV}(\mathrm{InP})$ and $2.25 \mathrm{eV}$ (AlP). In the work by Gagliano et al., AlInP NWs have been grown on (111)A InP substrates via SAG in an MOVPE reactor ${ }^{[129]}$ By controlling V/III ratio, NWs are crystallized into the pure WZ phase. These NWs present a high level of uniformity (Fig. 14a). They also exhibit complex morphology, with the hexagonal cross-section of pure InP NWs transforming into dodecagonal cross section upon increasing Al content (Figs. 14b-d). This change is induced by the alterations in V/III ratio and elemental composition, which influenced the growth rate of the different families of side facets of AlInP NWs. It is interesting to notice that an Al-rich AlInP inner shell is spontaneously formed, which is triggered by the slanted side facets of AlInP NWs. ${ }^{[129]}$ Additionally, owing to its low diffusivity, Al tends to segregate at the edges between the facets, forming Al-rich lines that are radially connected to the inner shell. The PL peaks shift towards the green region with increasing $\mathrm{Al}$ content and the maximum $\mathrm{Al}$ content that was reached is $40 \%$ (Fig. 14e). It is highlighted that this high level of confinement leads to increased emission lifetime of $0.5 \mathrm{~ns}-$ $1.5 \mathrm{~ns}$ at low temperatures, while at room temperature the lifetime is much lower, reaching the value of 0.3 ns. $^{[129]}$

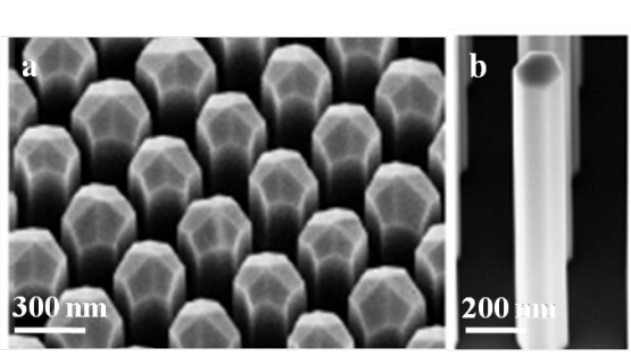

WZ InP NWs

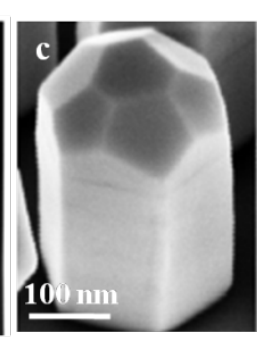

$\mathrm{WZ} \mathrm{Al}_{0.15} \mathrm{In}_{0.85} \mathrm{P}$ NWs

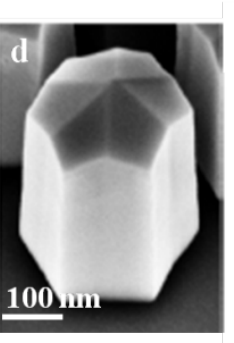

$\mathrm{WZ} \mathrm{Al}_{0.25} \mathrm{In}_{\mathbf{0 . 7 5}} \mathrm{P}$ NWs

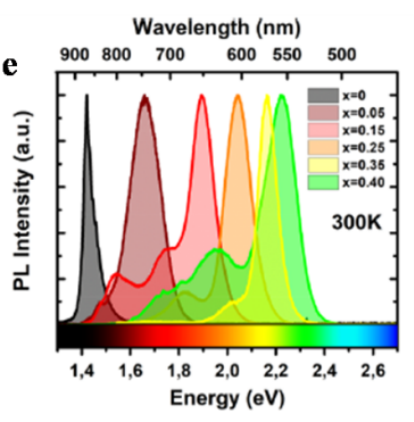

Figure 14: a) SEM image of an AlInP NW array, demonstrating a high degree of morphological homogeneity. $b$-d) High magnification SEM images of a WZ InP NW and two WZ AlInP NWs with Al content at $15 \%$ and $25 \%$, respectively. Increasing Al content leads to a transition of the initial, hexagonal cross section to a more complex dodecagonal form, potentially due to the alterations of V/III ratio and elemental composition. e) PL spectra of AlInP NW ensembles. Increasing Al content induces a blue shift of the peak towards the green region of the spectrum. For 0\%-40\% Al, emission covers the range between $875 \mathrm{~nm}$ and $555 \mathrm{~nm}$ (infrared to green region). (Figs. 14a-e: Reprinted with permission from ref. 129. Copyright (2018) American Chemical Society. Further permissions related to the material excerpted should be directed to the ACS.) 
After having thoroughly analysed the literature for the growth of ternary III-V NWs on Si and having included some particularly interesting cases of growth on different substrates, as a next step we will focus on the devices based on the ternary NWs. These devices include lasers, solar cells, water splitting, photodetectors and FETs and will be briefly described in different subchapters of the following section.

\section{5) Devices based on ternary III-V NWs}

\section{1) Lasers}

Group III-V semiconductor materials are ideal candidates for the fabrication of lasers, which produce stimulated emission at a desirable wavelength at room temperature. Lasers have been fabricated by employing QWs and QDs with excellent results. However, the lattice mismatch of the III-V compounds and Si substrates that induce strain can lead to defects such as threading dislocations, which enhance non-radiative recombination and hinder the performance of the devices. NW geometry allows for their monolithic integration on the mature Si platform, with elastic strain relaxation and lack of threading dislocations. For that reason, semiconductor NW lasers have been extensively studied as one of the emerging nanolasers. Another advantage for NW lasing is the necessary Fabry-Perot (FP) cavity to achieve stimulated emission as itis intrinsically formed in the micrometre scale by the NW geometry, which is different from the deliberate FP cavities by etching in traditional laser structure. NW lasers have been realized in various NWs-based nanostructures such as bulk NW material, dot-in-wire approaches and core/shell NWs with additional QWs in the shell as the active region. More specifically, group III-V GaAs, GaSb and phosphides can push the lasing to the infrared region, which is ideal for applications in optical communications, biomedical applications, and information technologies. Nevertheless, the lack of tunability of the bandgap and the wavelength of the stimulated emission is a significant obstacle in the wider implementation of binary III-V NW lasers. On another hand, ternary NWs offer a large range of tunability of their bandgaps, along with controllable material quality, which enables tuning of lasing to the desired region of the spectrum.

As mentioned in the previous section, the combination of GaAs core and AlGaAs shell is prevalent for laser fabrication, owing to the lattice match between the two compounds and to the bandgap of AlGaAs, which can be tuned in the near-infrared region. AlGaAs shell has a triple role; protecting the core from oxidation, neutralizing surface states, and confining the carriers in the core region. ${ }^{[97]}$ GaAs/AlGaAs NWs in laser fabrication exhibit good features up to room temperature. ${ }^{[100-101]}$ In addition, GaAs/AlGaAs core/shell NWs as lasers have exhibited characteristic temperatures up to $109 \pm 12 \mathrm{~K} .{ }^{[130]}$ Besides, lasing at the near-infrared region at a threshold of approximately $179 \mu \mathrm{J} / \mathrm{cm}^{2}$ has been revealed by embedding $50 \mathrm{InGaAs}$ QDs in the axis of GaAs/AlGaAs core/shell NWs grown on GaAs (111)B substrates. ${ }^{[110]}$ The emission was tuneable by controlling the size of the dots, even though the range of tunability is limited. Other than GaAs/AlGaAs core/shell NWs, different material combinations have 
been employed for the realization of lasers as well. For example, InAsP QDs embedded in InP NWs grown on InP (111)B substrates have also shown advantageous characteristics for laser fabrication, namely the high Q-factor and a record of eightfold enhancement of the emission. ${ }^{[131]}$ Enhanced stimulated emission and satisfying characteristics are exhibited in core/shell structures, where multiQWs are embedded in the shell. Low-threshold and room temperature operation have been exhibited in GaAs/AlGaAs core/shell NWs, with GaAs QWs embedded in the shell, a structure whose threshold was ranged at approximately $110 \mu \mathrm{J} / \mathrm{cm}^{2} /$ pulse. $^{[23]}$

On the other hand, on the matter of ternary core NWs on Si substrates, both GaAsSb and InGaAs have been exploited for laser fabrication. ${ }^{[109,132]}$ A challenge that is met in this procedure is the tunability of the wavelength of stimulated emission, low threshold and ability to operate up to room temperature. To overcome these obstacles, various material combinations and techniques have been reported. For instance, a structure composed of GaAsSb superlattices within GaAs NWs enabled lasing up to room temperature. ${ }^{[132]}$ Stimulated emission was observed at $950 \mathrm{~nm}$, with increasing pump power (Fig. 15a). It is also important to mention that tunability of the lasing peak was achieved between $990 \mathrm{~nm}$ and 890 $\mathrm{nm}$ by altering the $\mathrm{Sb}$ content. By reducing $\mathrm{Sb}$ content, the lasing peak blueshifts until it reaches 890 $\mathrm{nm}$ at $1 \% \mathrm{Sb}$ content (Fig. 15b). Besides, InGaAs/GaAs core/shell nanopillars also exhibited room temperature lasing, with the shell's thickness being a key factor for this function. ${ }^{[109]}$ Lasing was exhibited at $950 \mathrm{~nm}$, with a remarkably low threshold at $22 \mu \mathrm{J} / \mathrm{cm}^{2}$ (Fig. 15c). ${ }^{[109]}$ Most importantly, the wavelength of lasing was fully tunable by varying the In content of the core between $12 \%$ and $20 \%$, which enabled control of the wavelength of the stimulated emission from $890 \mathrm{~nm}$ to $940 \mathrm{~nm}$, approximately, upon In incorporation (Fig. 15d). ${ }^{[109]}$ Likewise, tuning of the lasing emission has been efficiently exhibited by controlling the In composition of InGaAs QWs in the shell of GaAs/(In,Al)GaAs core/shell NWs ${ }^{[133]}$ A cross-sectional TEM image (Fig. 15e) gives us a better insight of the structure. In this work by Stettner et al., low growth temperature for the shell and barrier layers between the QWs allowed In composition to reach 25\% without plastic relaxation or significant alloy intermixing, which in turn led to a full tunability of the lasing emission between the near-infrared and the telecommunications regions of the spectrum (Figs. $15 \mathrm{f}$ and $\mathrm{g}$ ). The lasing peak could be tuned by altering the In composition up to $40 \%$ at low temperature with the tuning range between $1.57 \mathrm{eV}$ and $1.36 \mathrm{eV}$ (Fig. 15f). The maximum In composition that demonstrated lasing at room temperatures was $30 \%$ (Fig. 15g). ${ }^{[133]}$

An important amount of work has been the realization of InGaAs NWs or nanopillars on silicon-oninsulator (SOI) substrates via SAG. ${ }^{[134-136]}$ The NWs exhibit a wide tunability of their composition, which leads to the emission being fully controllable and able to cover both the $1.31 \mu \mathrm{m}$ and the 1.55 $\mu \mathrm{m}$ telecommunication windows (Fig. 15h). ${ }^{[135]}$ In addition, a novel morphology where emission from a single InGaAs NW coupled into an SOI waveguide is a gaping step towards highly efficient optical communications. ${ }^{[135]}$ InGaAs/InGaP core/shell NW arrays have also been fabricated on SOI for lasing. ${ }^{[134]}$ Room temperature lasing has been achieved via optical pumping, and the range of the lasing 
wavelength could be tuned between $1100 \mathrm{~nm}$ and $1440 \mathrm{~nm}$, covering the important telecommunication window of $1.31 \mu \mathrm{m}$. Photonic crystal cavity formed by InGaAs nanopillars have also been fabricated on SOI, achieving strong horizontal confinement and lasing emission between $1020 \mathrm{~nm}$ and $1300 \mathrm{~nm}$ merely by altering the dimensions of the pitch or the diameter of the structures. ${ }^{[136]}$
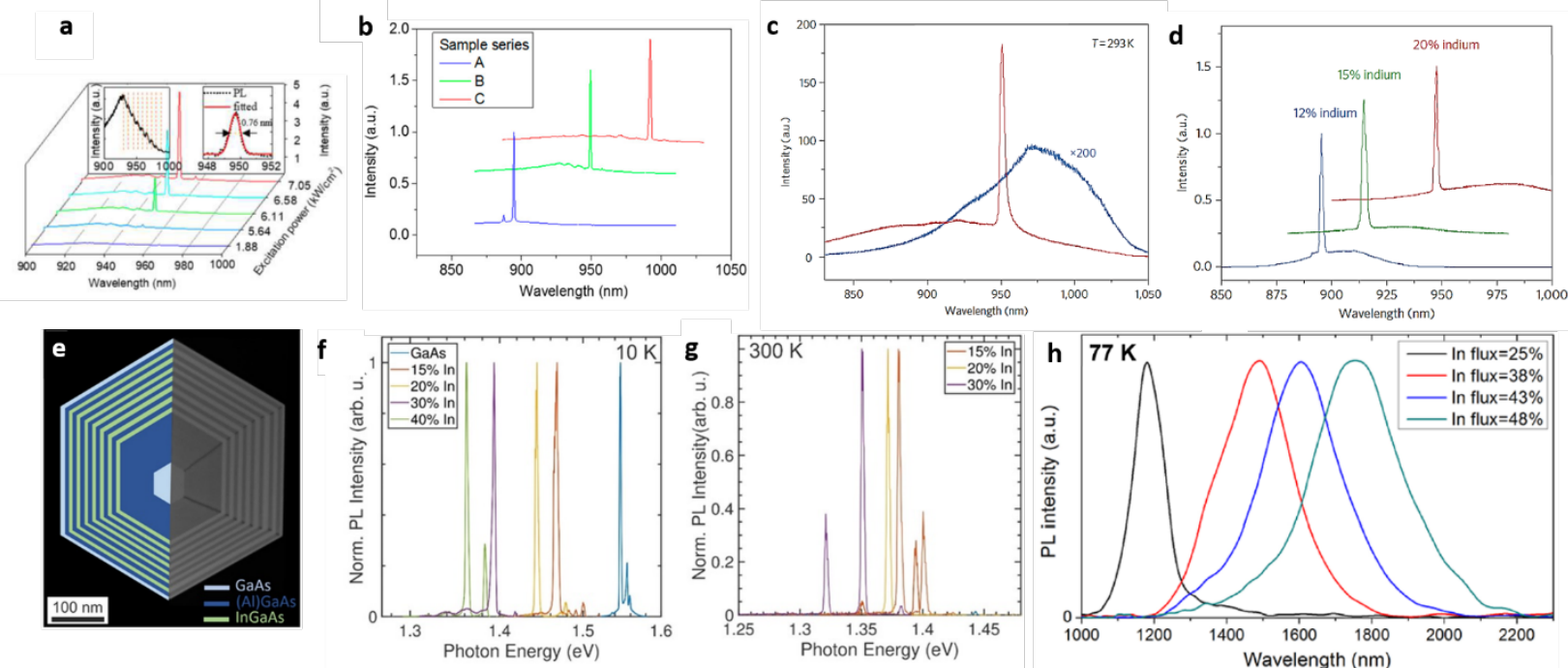

Figure 15: a) PL spectra at room temperature for different excitation powers. The insets show a PL spectrum of FP modes below lasing threshold under a pump power density of $1.88 \mathrm{~kW} / \mathrm{cm}^{2}$ (left) and a lasing peak with a linewidth of $0.76 \mathrm{~nm}$ (right). b) PL specra of three different samples with Sb compositions of $1 \%, 5 \%$ and $8 \%$ for samples A, B and C, respectively. Increasing Sb presence leads to a redshift of the lasing peak enabling tunability between $890 \mathrm{~nm}$ and $990 \mathrm{~nm}$. (Figs. 15a-b: Reprinted with permission from ref. 132. Copyright (2018) American Chemical Society) c) Lasing from an InGaAs nanopillars surrounded by GaAs shell, at $950 \mathrm{~nm}$. The device operated up to room temperature (293 K). d) PL spectra from InGaAs/GaAs core/shell nanopillars exhibiting lasing at different wavelengths as a function of the nominal composition of In. The In content of the core was varied between $12 \%$ and $20 \%$ leading to a control of lasing between $890 \mathrm{~nm}$ and $940 \mathrm{~nm}$. (Figs. $15 \mathrm{c}-d$ : Reprinted by permission from Springer Nature: Nature Photonics, ref. 109. Copyright (2011)) e) Cross-sectional TEM image of a GaAs/(In,Al)GaAs core/shell NW with InGaAs QWs embedded in the shell. f) PL spectra of the structure of Fig. 15e at $10 \mathrm{~K}$, exhibiting lasing with tunability from $1.36 \mathrm{eV}$ to $1.57 \mathrm{eV}$ for In composition ranging between 0 and $40 \%$ g) Similar spectra at room temperature exhibiting tunability up to $30 \%$ In content. (Figs. 15e-g: Reprinted with permission from ref. 133. Copyright (2018) American Chemical Society) h) PL spectra from InGaAs NW grown on SOI exhibiting tuneable emission, covering $1.33 \mu \mathrm{m}$ and $1.51 \mu \mathrm{m}$ telecommunication windows via tuning of their In composition. (Fig. 15h: Reprinted with permission from ref. 135. Copyright (2016) American Chemical Society) 


\section{2) Solar Cells}

Solar energy is a particularly attractive and alternative, energy source since it is abundant and clean, without causing any pollution to the environment. It can be converted into electrical energy with multiple benefits regarding financial and environmental issues. Due to these advantageous features and the limitations of the natural resources from where energy is harvested, efforts are being made in increasing the cost efficiency and operational efficiency of such devices. However, the efficiency of the fabricated devices remains low in the order of $9 \%-18 \%{ }^{[137]}$ It is noted the efficiency of tandem solar cells can reach a higher value. Consequently, new material platforms with higher efficiency are currently under intense investigation. The direct bandgap of group III-V semiconductors, along with their high absorption, render them ideal for this objective on Si which is the dominant platform on which most solar cells are based as it is widely explored and cheap. On that matter, NWs are beneficial due to their ability to be monolithically integrated with $\mathrm{Si}$ and their enhanced light extraction efficiency. ${ }^{[138]}$ Moreover, the formation of ternary alloys allows for a bandgap tunability, which is crucial as the bandgap of the materials needs to be small enough to absorb a wide range of the solar spectrum..$^{[22]}$ Indeed, NW array solar cells can theoretically achieve almost ideal light absorption with minimal material consumption. ${ }^{[137]}$ Therefore, ternary III-V NWs on Si have been systematically employed for solar cell fabrication via different approaches.

As mentioned in the previous section, $\mathrm{GaP}$ has an ideal bandgap and band alignment for solar energy conversion. Additionally, the bandgap of GaAsP becomes direct at $\mathrm{P}$ content less than $44 \%{ }^{[65]}$ The radial p-i-n junction, which is vital for solar energy conversion, occurs via opposite doping of the core and shell of the NW. ${ }^{[139]}$ The p-n junction can also be formed at the interface between the NW and the substrate. However, it is noted that since the radial p-n junction is long in the direction of the incident light and thin in the orthogonal direction, it combines the benefits of enhanced light absorption and effective carrier collection. Besides, GaAsP NWs may include a passivation layer with remarkable effects on the efficiency of the solar cells. In ref. 140, InGaAs has been used for surface passivation with notable results in the efficiency of the fabricated solar cells. The best passivated device is depicted in the SEM of Fig. 16a, for which the increase in the efficiency was $72 \%$, increasing the conversion efficiency above $10 \%$ (Fig. 16b). Besides the open circuit voltage was increased from $0.7 \mathrm{~V}$ to $0.9 \mathrm{~V}$. These improvements were attributed to the passivation layer prohibiting the carriers from reaching the surface and recombine non-radiatively.

InGaAs NWs have also been used as the major platform for solar cell fabrication. In ref. 20, solar cells has been fabricated based on the intrinsic p-n junction, where Si substrate is p-type and NW is ntype (Fig. 16c). An InAlAs shell is grown surrounding the InGaAs core, with remarkable results in the photovoltaic performance of the devices. The short circuit current increases from $4.3 \mu \mathrm{A} / \mathrm{cm}^{2}$ to 10 $\mu \mathrm{A} / \mathrm{cm}^{2}{ }^{2}{ }^{[20]}$ It is important to notice that the upper section of the shell is etched, to ensure good Ohmic contacts. The I-V curve of the device under dark exhibits a highly rectifying behaviour, similar to a 
diode (Fig. 16d). The unpassivated NWs have an I-V curve that exhibits an S-shape behaviour, indicative of a low conversion efficiency due to losses attributed to surface states and defects. On the contrary, the lack of the S-shape behaviour for the core/shell NWs highlights their superiority (Fig. $16 \mathrm{e}) .{ }^{[20]}$

A similar device structure has been adopted for InAsP NWs grown on Si as well. ${ }^{[21]}$ In this case, the p-n junction is intrinsically formed between the Si substrate and the InAsP NWs. It is noted that no parasitic film is grown, which implies that the p-n junction is purely formed between the p-Si and nInAsP. The I-V curve of the solar cells shows highly rectifying behaviour (Fig. 16f). ${ }^{[21]}$ The high rectifying ratio along with the low reversed bias leakage current are indicative for a type II band alignment, where the conduction band and the valence band of Si are located above the ones of InAsP. Another factor examined is the ideality factor, which is a value varying between 1 and 2 and takes the value of 1 for ideal diodes. The ideality factor accounts for imperfections in the fabrication of real devices. In this case the ideality factor is very small which is 1.4 , much improved when compared to other NWs and can be compared to the one of state-of-the-arts NW homojunctions. ${ }^{[21]}$ As in the previous cases, the addition of an InP shell surrounding the InAsP core leads to spatial separation of the carriers from the surface, resulting in reduced non-radiative recombination and thus to a higher short circuit current and enhanced efficiency. ${ }^{[21]}$

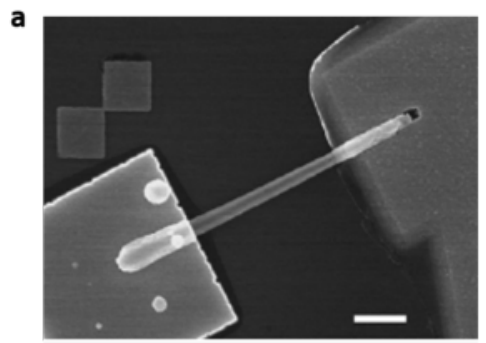

d

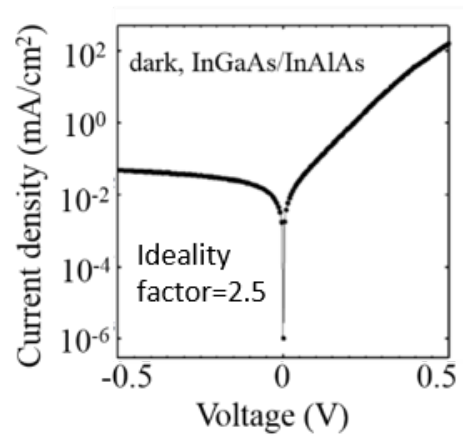

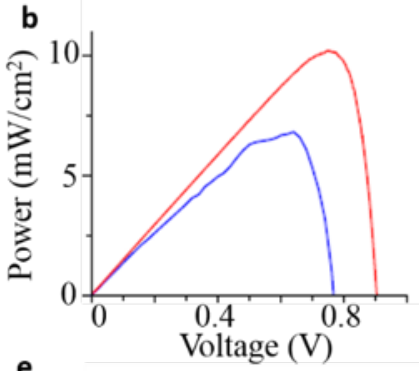

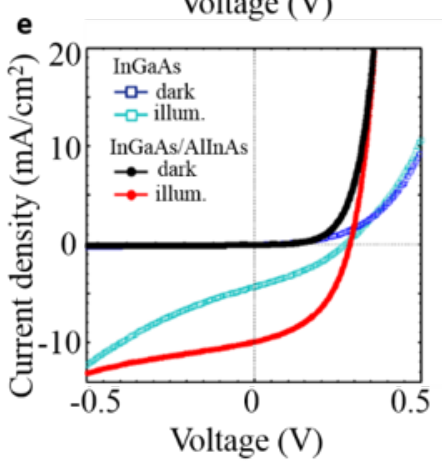

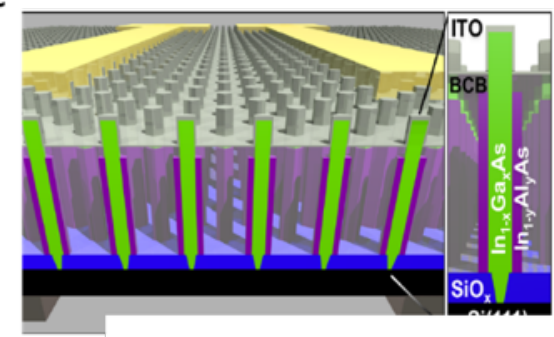

f

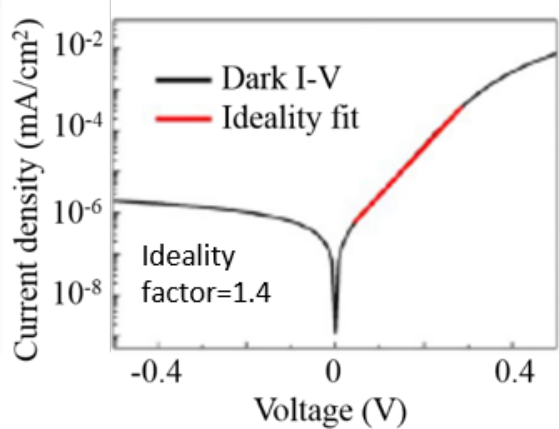

Figure 16: a) SEM of a GaAsP NW solar cell with InGaAs passivation layer. The scale bar is $1 \mu \mathrm{m} . \mathrm{b}$ ) $P$-V comparison between the passivated (red) and the unpassivated (blue) GaAsP NWs. The power reaches a maximum of $10.2 \mathrm{~mW} / \mathrm{cm}^{2}$ for the passivated $N W s$ while remaining at $6.8 \mathrm{~mW} / \mathrm{cm}^{2}$ for unpassivated NWs. (Figs. 16a-b: Reprinted with permission by Springer Nature: Nature Communications, ref. 140. Copyright (2013)) c) Schematics of photovoltaic solar cell structures based 
on InGaAs/AlInAs NWs on Si. d) I-V curve in dark for InGaAs/AlInAs NWs showing a clear rectifying, diode-like behaviour. The ideality factor is extracted as 2.5. e) Comparison of the I-V curves in dark and under illumination for passivated and unpassivated NWs. The curve of the unpassivated NWs exhibits $S$-shape behaviour, indicative of losses and low efficiency, which is absent from core/shell NWs. (Figs. 16c-e: Reprinted with permission from ref. 20. Copyright (2015) American Chemical Society. Further permissions related to this material excerpted should be directed to the ACS.) f) I-V curve of solar cells based on InAsP NWs. Clear diode, rectifying behaviour is exhibited. The ideality factor is small at 1.4 and comparable to the up-to-date NW solar cell technologies. (Fig. 16f: Reprinted with permission from ref. 21. Copyright (2013) American Chemical Society)

\section{3) Water splitting devices}

Another ideal candidate source for green energy is hydrogen, as it is the simplest and most common compound in nature. One of the potential ways to develop such a technology is to convert solar energy to hydrogen via water splitting directly. Although this field has been intensively studied for many years, the results regarding the efficiency of this conversion are far from enough to implement it on its full scale. For photocathode fabrication, several wide bandgap materials have been preferred, especially $\mathrm{TiO}_{2}$ due to its photochemical stability. However, the bandgap of the material needs to be small enough to absorb a wide range of the solar spectrum and large enough to induce water splitting. The ideal values range between $1.6 \mathrm{eV}$ and $2.4 \mathrm{eV} \cdot{ }^{[22]}$ As a direct consequence, the use of direct bandgap III-V semiconductors is beneficial. Additionally, ternary III-V alloys offer the advantage of tuneable bandgap, which is also desirable.

GaAsP has a tuneable bandgap between $1.4 \mathrm{eV}$ and $2.26 \mathrm{eV}$, which renders it ideal for water splitting devices fabrication. In the work by Wu et al., the growth of GaAsP core/shell NWs was conducted monolithically on Si via solid-source MBE (Fig. 17a). ${ }^{[22]}$ The p-type core is Be-doped and the n-type shell is Si-doped. Due to the large surface to volume ratio in NW structures, the high density of surface states might hinder the efficiency of the resulting devices. As a result, surface passivation via the use of an InGaAs layer surrounding the core/shell homojunction can significantly improve the performance of the water splitting structures. ${ }^{[22]}$ The homogeneous NW array is then implemented in water splitting device fabrication, with the schematics of Fig. 17b depicting the configuration of the photocathodes. For the unpassivated structures, the photocurrent onset is $0.54 \mathrm{~V}$ vs. reversible hydrogen electrode (RHE), while the photocurrent density at $0 \mathrm{~V}$ vs. RHE is $4.5 \mu \mathrm{A} / \mathrm{cm}^{2}$ (Fig. 17c). It is noted that the photocurrent remains stable for $30 \mathrm{~min}$. With the addition of the InGaAs passivation layer, the surface states are neutralized and the carriers are confined within the NW. Consequently, the photocurrent density at $0 \mathrm{~V}$ vs. RHE is increased to $4.7 \mu \mathrm{A} / \mathrm{cm}^{2}$, while the photocurrent onset is at $0.63 \mathrm{~V}$ vs. RHE (Fig. 17d). The achieved solar to hydrogen (STH) conversion efficiency achieved is $0.5 \%$, much higher than the ones reported for other NW photocathodes. However, the photocurrent stability is deteriorated 
and remains stable for less than $10 \mathrm{~min}$, which is attributed to the rapid photochemical corrosion that the InGaAs passivation layer suffers. ${ }^{[22]}$

a

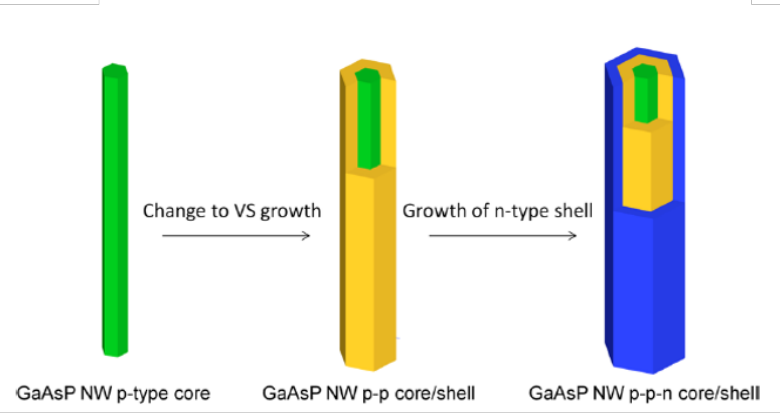

C

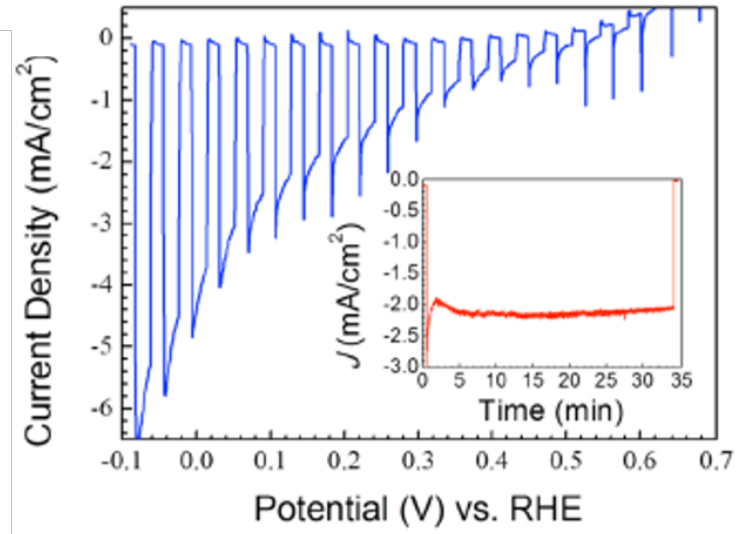

b

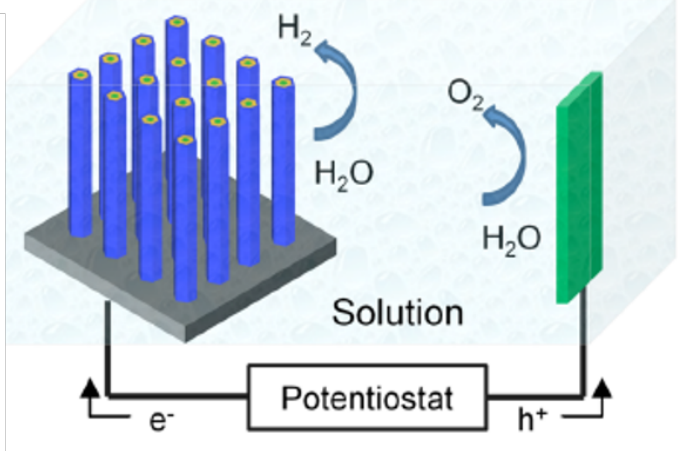

d

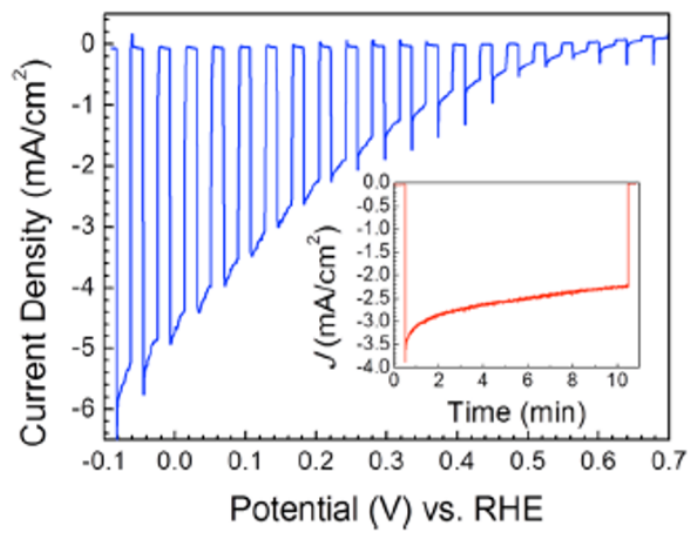

Figure 17: a) Schematics of the GaAsP NW, showing the growth of the p-type core and n-type shell, with an intrinsic shell between the two. b) Schematics of a water splitting device consisted of a NW array grown on Si substrates. c) Current density potential characteristics of GaAsP homojunction NWs photocathode. d) Current density potential characteristics of GaAsP NWs photocathode with the addition of an InGaAs passivation shell. The insets in Figs. 17c-d show the steady-state current density of the photocathodes. The measurements were conducted at $0.1 \mathrm{~V} v \mathrm{~s}$. RHE under AM 1.5G illumination. (Figs. 17a-d: Reprinted with permission from ref. 22. Copyright (2014) American Chemical Society. Further permissions related to the material excerpted should be directed to the ACS.)

\section{4) Photodetectors}

Photodetectors are light sensors, which include a p-n junction that enables the conversion of light into current. Group III-V semiconductors have excellent optical properties owing to some of their advantageous features, such as the direct bandgap. As a result, they have been frequently employed for 
the realization of photodetectors. Especially antimonides enable photodetection in the near- or midinfrared region of the spectrum, which is crucial for a wide range of applications regarding detection of infrared light. Ternary III-V alloys allow for a bandgap tuning, particularly beneficial for this type of optical applications. Considering the monolithic integration of III-Vs on $\mathrm{Si}$, offered by the unique NW geometry, ternary III-V NWs are attractive as platforms for the fabrication of photodetectors. In this part of the fifth section, we will briefly describe photodetectors based on ternary III-V NWs on Si.

GaAsSb has been implemented for the fabrication of near-infrared photodetectors with excellent results. In the work by Huh et al., the single NW-based photodetector was organized in a four-probe geometry as depicted in the SEM of Fig. $18 \mathrm{a} \cdot{ }^{[79]}$ From, the I-V curves, the first observation is a clear rectifying behaviour of single GaAsSb NWs after device fabrication (Fig. 18b). ${ }^{[27,79]}$ The rectifying behaviour is attributed to the trend of the uneven distribution of $\mathrm{Sb}$, with several fluctuations in the NW, which creates asymmetric Schottky contacts between the NW edges and the metal contacts. Additionally, $\mathrm{Sb}$ is diffused at the NW surface and forms Sb vacancies via an As-Sb exchange. ${ }^{[79]}$ These defects act as acceptors and can contribute in the rectifying behaviour, as well. ${ }^{[27]}$ In ref. 79 , the photoresponsivity achieved at reverse bias and under illumination is $1463 \mathrm{~A} / \mathrm{W}$, a value 48,000 times higher than the one reported for GaAs NW-based photodetectors and comparable to the state-of-the-art nanostructure-based photodetectors (Fig. 18c). It is ascribed to the higher electric field for a reversely biased Schottky barrier. On the other hand, the rise time and decay time are relatively large at $32 \mathrm{~ms}$ and $136 \mathrm{~ms}$, respectively, signifying the persistence of the photocurrent, due to Sb-related defects. ${ }^{[79]} \mathrm{In}$ addition, high $\mathrm{Sb}$ content has a profound influence on the performance of photodetectors, as well. It is observed that in InAsSb NW-based photodetectors, the presence of $\mathrm{Sb}$ and the resulting high-quality material and $\mathrm{Sb}$ accumulation at the external facets caused a record-low leakage current that enabled a better performance of the device. ${ }^{[95]}$ Moreover, this design caused electrical conduction through the $\mathrm{Si}$ substrates, which renders potential integration very promising. ${ }^{[95]}$

After having examined single NW-based photodetectors it is important to examine the figures of merit and the performance in an ensemble photodetector. The NWs in this case include an AlGaAs shell that is used for surface passivation (Fig. 18d). ${ }^{[28]}$ In the I-V curves, rectifying behaviour is observed due to the formation of asymmetric Schottky contacts at the ends of the NWs (Fig. 18e). The photoresponsivity under illumination at reverse bias is $311 \mathrm{~A} / \mathrm{W}$. It is noted that as-grown NWs result in devices with large dark currents and low photocurrents, with approximate values of $10^{-4} \mathrm{~A}$ and $10^{-3}$ A, respectively at the forward bias. However, in-situ annealing after growth improves the performance of the photodetectors resulting in a higher photocurrent of more than $10^{-2} \mathrm{~A}$ and a lower dark current of $10^{-5} \mathrm{~A}$ at the forward bias (Fig. 18f). Despite the high carrier mobility presented in this case, the results could not reach the level of single NW-based photodetectors. ${ }^{[28]}$ 


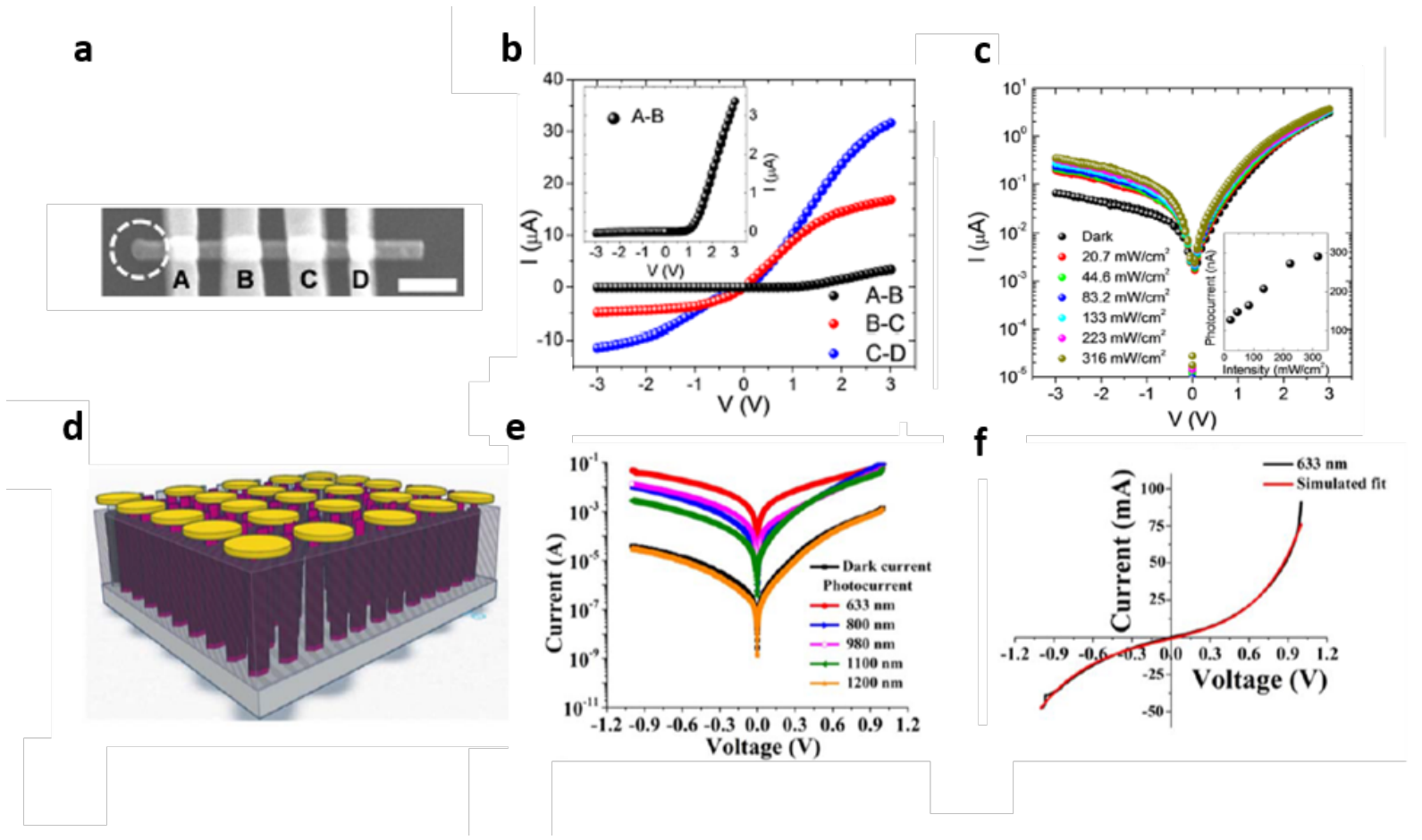

Figure 18: a) SEM image of a single GaAsSb NW-based device with four metal electrodes. The scale bar is $500 \mathrm{~nm}$. b) I-V curve of a single GaAsSb NW in four-probe geometry (A-D). Probe A is close to the tip and $D$ is close to the base as viewed in the SEM image of Fig. 18a. Rectifying behaviour is clearly observed in all configurations (with the C-D measurement closer to the base being more pronounced). c) I-V curves under different illuminations of the photodetector fabricated by GaAsSb NWs that gave the measurements of Fig. 18b. The rectifying behaviour observed is a result of the asymmetric Schottky contacts formed at the NW ends. The inset shows the photocurrent as a function of the intensity of illumination. The photoresponsivity is calculated as $1463 \mathrm{~A} / \mathrm{W}$. (Figs. 18a-c: Reprinted with permission from ref. 79. Copyright (2015) American Chemical Society) d) Schematics of a photodetector based on an array of GaAsSb/AlGaAs core/shell NWs. e) I-V curves of the photodetector of Fig. 18d under different illuminations. Rectifying behaviour indicates the existence of asymmetric Schottky contacts at both NW ends. f) I-V curve of the device of Fig. 18d. The photocurrent has a high value at forward bias, due to previous $N W$ annealing. The stimulated fit is depicted by the red line. (Figs. 18d-f: Reprinted with permission from ref. 28. Copyright (2018) IOP Publishing Ltd.)

\section{5) Field-effect transistors (FETs)}

The implementation of group III-V semiconductors in various types of FETs is gaining more and more attention, mainly because of the high carrier mobility of this category of materials. The dominance of electronics in an abundance of applications render investigation of this field crucial. The key to the exploitation of the advantageous carrier transport properties of III-V materials, however, is their onchip integration. Considering that the vast majority of electronics is based on the cheap Si platform, 
monolithic growth of III-V structures on $\mathrm{Si}$ is a priority for the realization of efficient FETs. Until recently, this procedure was hindered by the large lattice mismatch between III-Vs and Si. This obstacle can be overcome with the adoption of the NW geometry which, as mentioned extensively in the current paper, offers elastic strain relaxation, allows for monolithic integration with the Si platform and has the potential to improve the performance of the NW-based devices.

Electron transport properties of ternary III-V NWs are an exciting topic of research. Owing to the high carrier mobility it presents, InGaAs is one of the most attractive ternary alloys for FET fabrication. Back-gated FETs can be fabricated based on these NWs, by using metal contacts defined via lithography techniques (Fig. 19a). ${ }^{[32,111,141]}$ InGaAs NW FETs exhibit n-type conductivity. In the work by Hou et al., by investigating a single NW-based FET, The I I $/ \mathrm{I}_{\mathrm{OFF}}$ ratio is up to $10^{5}$ (Fig. 19b). ${ }^{[32]}$ The mobility is also calculated as a function of back-gate voltage $\mathrm{V}_{\mathrm{GS}}$, which is enhanced and reaches 2700 $\mathrm{cm}^{2} /\left(\mathrm{V}^{*} \mathrm{~s}\right)$, comparable to the state-of-the-art InAs NW-based FETs. However, for similar measurements on FETs based on an ensemble of parallel InGaAs NWs, it greatly reduces the carrier mobility and the FET performance. The $\mathrm{I}_{\mathrm{ON}} / \mathrm{I}_{\mathrm{OFF}}$ ratio is 500 and the carrier mobility approximately 60 $\mathrm{cm}^{2} /(\mathrm{V} * \mathrm{~s})$ which is attributed to the non-optimized growth causing fractures or misalignments of the NW ensemble and creates parasitic capacitance. For that reason, chemical treatment could be used for surface passivation to improve the performance of the devices. ${ }^{[32]}$ It is noted that the dimensions of the $\mathrm{NWs}^{[141]}$ and the elemental composition of the alloy ${ }^{[111]}$ are important in the carrier mobility and, thus, the performance of the FETs. More specifically, the carrier mobility is reduced in smaller InGaAs NWs, as deduced by the I-V curves of devices based on different NWs (Fig. 19c). The possible reasons for this decrease are several, such as 1) the increased phonon and surface scattering of carriers in smaller NWs, and 2) the reduced effective channel width and less subbands, all of which result in a reduced normalized current. ${ }^{[141]}$ Finally, In-richer structures exhibit a vast increase in the carrier mobility, which also may decrease the turn off-ratio as leakage current may appear due to the reduction of the bandgap. ${ }^{[111]}$

InGaAs NWs have also been used for the fabrication of surround-gated FETs. ${ }^{[31]}$ It is noted that the advantage of surround-gated transistors is the ability to remove ungated regions. Despite the transport in these regions usually increases parasitic source and drain resistance and can degrade the performance of the metal-oxide-semiconductor field-effect transistor (MOSFET), the addition of the InGaAs region and its use as the drain increases the transconductance and reduces the subthreshold swing of the transistors. As a result, the leakage is much less and lower off-state currents can be obtained, without any degradation of the on-state performance of the transistor, and the lower leakage current leads to the operation of the transistor at higher voltages without suffering from a breakdown. ${ }^{[142]}$

A surround-gated or gate-all-around FET has been demonstrated by fabricating a parallel array of NWs, and the material comprising the gate is surrounding the NWs. The drain and source are comprised of metal contacts (Fig. 19d) ${ }^{[31]}$ Under such a configuration, the leakage current is minimal, and no noticeable hysteresis is found in the capacitance vs. gate voltage graph (Fig. 19e). The source-drain 
current is modulated by the gate voltage, with an $\mathrm{I}_{\mathrm{ON}} / \mathrm{I}_{\mathrm{OFF}}$ ratio of $10^{6}$ (Fig. 19f). Finally, the threshold voltage is $0.18 \mathrm{~V}$, and the peak transconductance at $\mathrm{V}_{\mathrm{DS}}$ of $1 \mathrm{~V}$ is $280 \mu \mathrm{S} / \mu \mathrm{m} .{ }^{[31]}$ The good switching characteristics have even more room for improvement, by passivation of surface states to increase carrier mobility within the channel. The adoption of the core/multishell structure described earlier, for instance, may lead to a vast increase in the transconductance and drain current at $1.42 \mathrm{mS} / \mu \mathrm{m}$ and 45 $\mu \mathrm{A} / \mu \mathrm{m}$, respectively. The IoN $/ \mathrm{I}_{\mathrm{OFF}}$ ratio is increased to $10^{8}$, indicative of the excellent switching features of the FETs. ${ }^{[31]}$
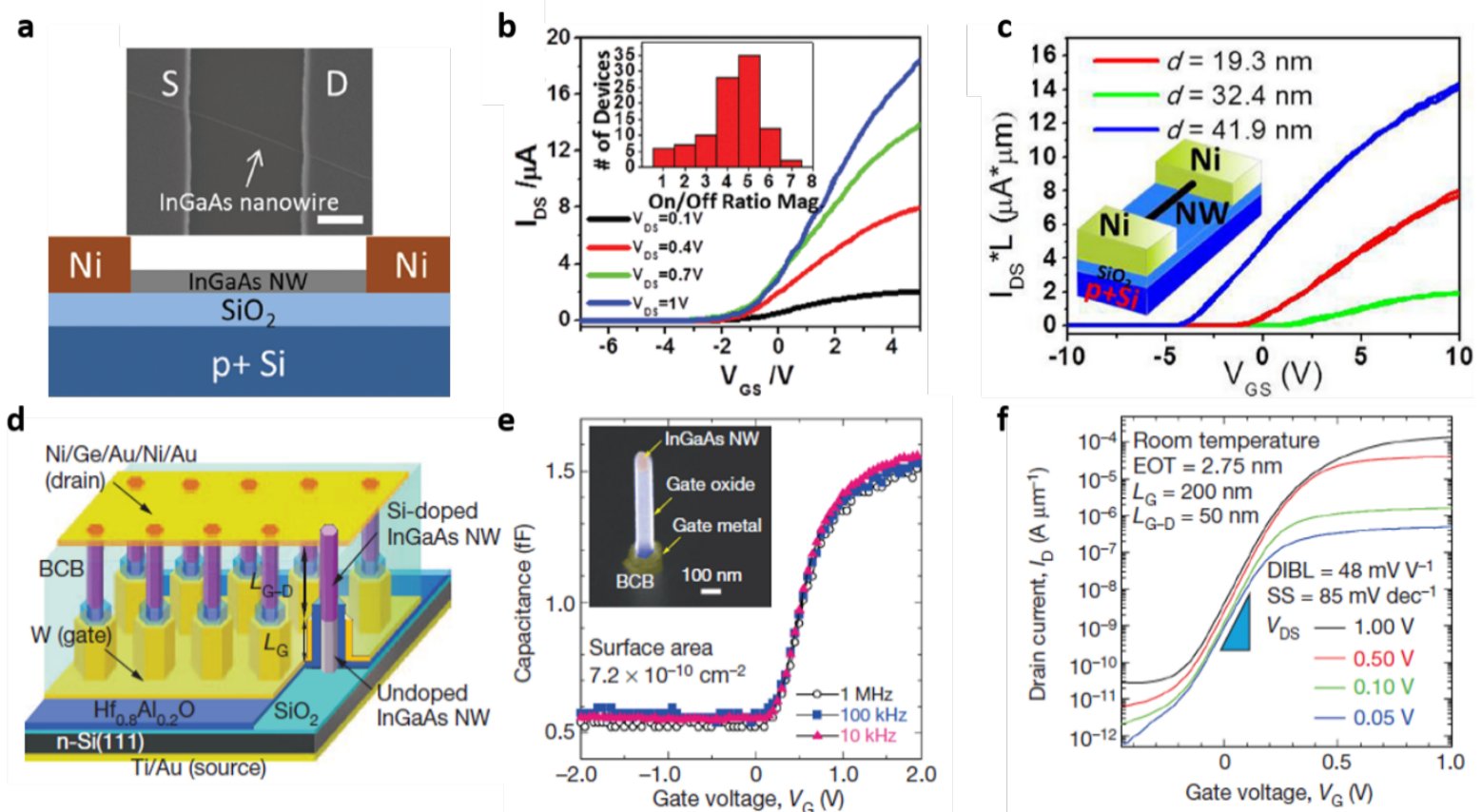

Figure 19: a) SEM (top) and schematics (bottom) of a back-gated FET with metal contacts based on a single InGaAs NW. b) Transfer characteristics of the FET of Fig. 19 a for different $V_{D S}$. The inset depicts the statistics of the $I_{O N} / I_{\text {OFF }}$ ratio of 100 such devices. (Figs. 19a-b: Reprinted with permission from ref. 32. Copyright (2012) American Chemical Society c) Transfer characteristics of three back-gated FETs based on single In $n_{0.7} G a_{0.3} A s$ NWs with three different diameters. Smaller NWs present reduced electron transport and mobility. (Fig. 19c: Reprinted ref. 141 with the permission of AIP publishing.) d) Surround-gated FET comprised of a parallel array of 10 InGaAs NWs. Each NW is composed of a Sidoped InGaAs on top of an undoped In GaAs $\mathrm{NW}$. Each $\mathrm{NW}$ is wrapped with $\mathrm{Hf}_{0.8} \mathrm{Al}_{0.2} \mathrm{O}$ gate oxide and tungsten (W) gate metal. $L_{G}$ is $200 \mathrm{~nm}$ and $L_{G-D}$ is $50 \mathrm{~nm}$. e) Capacitance-gate voltage curve of the surround gated FET of Fig. 19d, with 250 parallel NWs. The inset shows the SEM of a representative structure. f) Transfer characteristics of a surround-gated FET based on InGaAs NWs. The values of different parameters are included in the Figure. (Figs. 19d-f: Reprinted by permission from Springer Nature: Nature, ref. 31. Copyright (2012)) 
The high carrier mobility of InAs among the most of rest binary III-V semiconductors increased the interest for other ternary alloys comprised of InAs. For that reason, InAsSb NWs have also been examined for FET fabrication. ${ }^{[30]}$ The FETs constructed by InAsSb NWs exhibit a remarkably low resistivity, which is a sign of the significant increase in the electron mobility upon more $\mathrm{Sb}$ incorporation in the structures. More specifically, InAsSb NWs with $15 \% \mathrm{Sb}$ content exhibit a six-fold reduction of resistivity, when compared to pure InAs NWs ${ }^{[30]}$ The surfactant effect of Sb, improving the material quality of the NWs and reducing the density of stacking faults, is a contributing factor to this phenomenon. It is noted that the impact of temperature and $\mathrm{Sb}$ concentration on the performance of the FET is strong and the difference is even more pronounced at Sb-richer structures. ${ }^{[30]}$

\section{6) Conclusion and Discussion}

Growth of III-V ternary NWs has attracted attention, due to their promising properties in bandgap engineering, controllable material quality and monolithic integration on the Si platform. In our literature review the recent advances have been discussed in the development of NWs and NWQDs based on III$\mathrm{V}$ ternary alloys on Si substrates, along with the challenges that were met. As described in the previous sections, one of the basic requirements is the realization of pure, defect-free NW structures, as crystal defects give rise to unwanted effects and uncontrollable phenomena. Indeed, as clearly shown in the case of $\mathrm{Sb}$, inhomogeneous elemental distribution can significantly alter the morphology and the properties of NWs and degrade the performance of the resulting devices. For instance, the photodetectors based on antimonides presented persistent photocurrent, attributed to Sb-related defects, such as antisites or vacancies. ${ }^{[79]} \mathrm{Sb}$ content also has a noticeable effect on the carrier mobility in InAsSb-based FETs. ${ }^{[30]}$ On the other hand, homogeneity in the morphology of the structures is a significant factor, especially for solar cells and other energy harvesting applications. ${ }^{[21-22,140]}$ Uniformity needs to be maintained in the elemental distribution of III-V elements within the NWs as well. Inhomogeneity in the elemental distribution alters the crystal structure, the morphology and the bandgap of the materials, leading to modifications in their optical properties. As a result, the performance of optical applications, such as lasers, based on ternary III-V NWs can be degraded.

As stated in the growth mechanisms section, each primary technique employed for NW growth exhibits its own advantages and disadvantages. SAG can potentially lead to a highly homogeneous array of vertically aligned NWs, which is beneficial to photovoltaic cells, but includes several additional steps of lithography and etching that increase the complexity and the cost of the procedure. ${ }^{[35]}$ On the contrary, Au-catalysed VLS growth is a direct approach with fewer steps and more straightforward in its realization. ${ }^{[39]}$ The formation of a liquid alloy among III-V adatoms and Au seeds is simple, due to the intrinsic properties of $\mathrm{Au}^{[40]}$ However, the control of the homogeneity in the elemental distribution is a challenging task, while Au is also a contaminant for the material system, as it can be alloyed with the Si substrate or diffuse into the NW forming deep trap energy states. ${ }^{[41]}$ Self-catalysed VLS growth has 
emerged as a promising growth technique, since group III droplets do not pose any contamination hazards and their post-growth consumption is accessible under a high group V flux. However, the reservoir effect hinders the abruptness of the interfaces between different parts of the NW, an effect that can also be detrimental to the functionality of the resulting NW-based devices. ${ }^{[42]}$ An alternative that has been exhibited is the catalyst-free approach, where the NWs are elongated as a result of the strain between III-V compounds and $\mathrm{Si}^{[49-50]}$ Nevertheless, this approach has not yet been thoroughly investigated and its exact mechanism is not fully comprehended. Consequently, control over the catalyst-free grown NWs is still at a relatively low level.

Despite the difficulties and the complexity of the procedures regarding the growth of ternary III-V NWs on $\mathrm{Si}$, the progress in device fabrication has also been significant. Energy harvesting devices such as solar cells and photocathodes for water splitting have shown promising characteristics and higher power conversion efficiency, when employing ternary III-V NWs. Even though room for improvement is still significant, the breakthroughs on this scientific field rendered the renewable energy sources and the alternatives to fossil fuels a realistic target for the future. Optoelectronic devices such as lasers and photodetectors based on ternary III-V NWs on Si, also show exceptional features. For instance, optically-pumped operation of ternary III-V nanolasers has been achieved at room temperature with lasing at tuneable wavelength. The controlled bandgap of III-V NWs enabled emission and photodetection of infrared light, a function that can be applied in several fields, such as telecommunications and medical applications. Furthermore, monolithic integration of III-V semiconductor NWs on the Si platform opened the route for the fabrication of highly efficient electronics that exhibit stability and good carrier transport features, with lower power consumption.

Our work has summarized and described the growth methods, properties and main applications of ternary III-V NWs on Si and briefly introduced ternary III-V NWs on other substrates. For clarity, in the following figure (Fig. 20), we present three tables, where the bandgap, growth methods, basic properties and device implementation of all the analysed ternary NWs are synopsised. Table I (Fig. 20a) contains III-V-V NWs, Table II (Fig. 20b) includes III-III-V NWs and the final table (Fig. 20c) encloses the four rare alloys that were described. It is reminded that, for the last case, growth on Si has not been reported yet. 


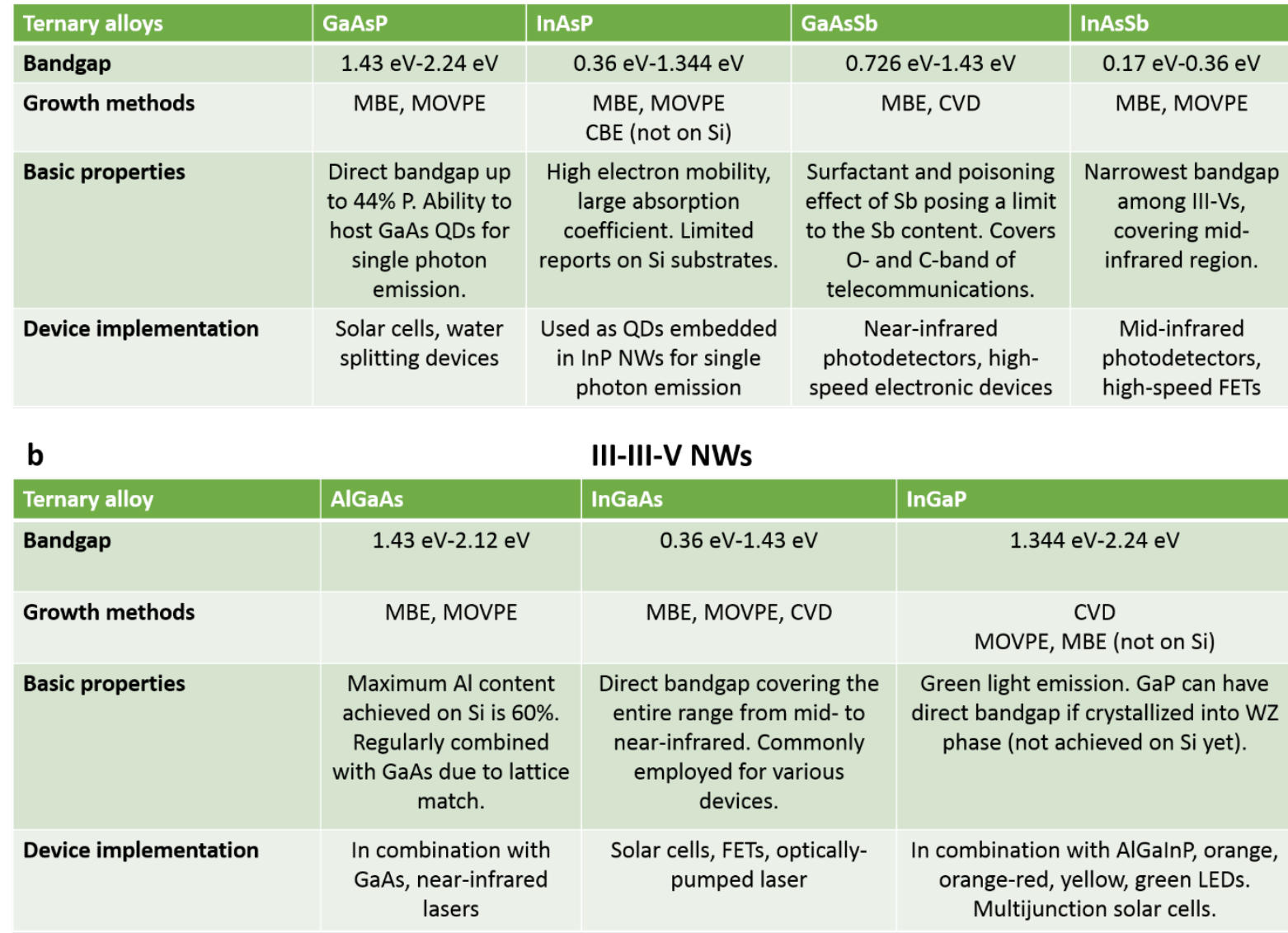

C

Rare NWs

\begin{tabular}{|c|c|c|c|c|}
\hline Ternary alloys & InGaSb & InPSb & GaPSb & AllnP \\
\hline Bandgap & $0.17 \mathrm{eV}-0.726 \mathrm{eV}$ & $0.17 \mathrm{eV}-1.344 \mathrm{eV}$ & $0.726 \mathrm{eV}-2.24 \mathrm{eV}$ & $1.344 \mathrm{eV}-2.25 \mathrm{eV}$ \\
\hline Growth methods & MOVPE (not on Si) & MOVPE (not on Si) & Reactive Vapour Transport (not on Si) & MOVPE (not on Si) \\
\hline Basic properties & $\begin{array}{l}\text { InSb has the } \\
\text { highest electron } \\
\text { mobility and GaSb } \\
\text { the highest hole } \\
\text { mobility among III- } \\
\text { V semiconductors. }\end{array}$ & $\begin{array}{l}\text { Highly tuneable } \\
\text { morphology. } \\
\text { Bandgap can cover } \\
\text { mid-infrared } \\
\text { region. }\end{array}$ & $\begin{array}{l}\text { Alloying of GaP with GaSb caused an } \\
\text { indirect-to-direct transition of the } \\
\text { bandgap. }\end{array}$ & $\begin{array}{l}\text { Direct bandgap in } \\
\text { the green region, } \\
\text { solution for the } \\
\text { 'green gap'. }\end{array}$ \\
\hline
\end{tabular}

Figure 20: a-c) Tables including III-V-V, III-III-V and rare alloys, respectively, encapsulating the bandgap, growth methods, basic properties and device implementation of the ternary III-V NWs described throughout the current paper.

To sum up, in the current review we have thoroughly analysed the growth methods and characterization of ternary III-V NWs on Si substrates and highlighted the unique properties and behaviours of each of the examined ternary alloys. By employing this type of alloys, the bandgap can be widely controlled and as a result the wavelength of the emission is tuneable between the values of the two binary alloys comprising the ternary material. The introduction of a third element in the ternary alloy frequently has a significant impact on the morphology and the crystal structure of the NWs. Some rare alloys grown on substrates other than Si have also been described. Based on these observations, 
the devices based on ternary III-V NWs on Si including lasers, solar cells, water splitting devices, photodetectors and FETs have been illuminated, with emphasis on their optimized performance. Even though there is a long path of refinements lying ahead, the investigation and advances in ternary III-V $\mathrm{NW}$ growth on $\mathrm{Si}$, presented in this paper, are promising for the future realization of highly efficient and accessible technologies in the field of electronics and optoelectronics.

\section{AUTHOR INFORMATION:}

\section{Corresponding author}

*Email: xuezhe.yu@ucl.ac.uk

Author Contribution

" G.Boras and X.Z. Yu contributed equally to this work.

Notes

The authors declare no competing financial interest.

\section{Acknowledgment:}

This project has received funding from the European Union's Horizon 2020 research and innovation programme under the Marie Skłodowska-Curie grant agreement No 721394. 


\section{References}

(1) Q.-Y. Tong, U. Gösele, Semiconductor Wafer Bonding, Science and Technology, John Wiley \& Sons, 1999, 204

(2) S. Palit, J. Kirch, M. Huang, L. Mawst, N. M. Jokerst, Facet-embedded thin-film III-V edge-emitting lasers integrated with SU-8 waveguides on silicon, Optics Letters, 2010, 35(20), 3474-3476

(3) S. Palit, J. Kirch, G. Tsvid, L. Mawst, T. Kuech, N. M. Jokerst, Low-threshold thin-film III-V lasers bonded to silicon with front and back side defined features, Optics Letters, 2009, 34(18), 2802-2804

(4) M. V. Bogdanov, K. A. Bulashevich, O. V. Khokhlev, I. Y. Evstratov, M. S. Ramm, S. Y. Karpov, Current crowding effect on light extraction efficiency of thin-film LEDs, Phys. Stat. Solidi (c), 2010, 7(7-8), 2124-2126

(5) J. J. Wierer Jr., A. David, M. M. Megens, III-nitride photonic-crystal light-emitting diodes with high extraction efficiency, Nature Photonics, 2009, 3, 163-169

(6) S. Pouladi, M. Rathi, D. Khatiwada, M. Asadirad, S. K. Oh, P. Dutta, Y. Yao, Y. Gao, S. Sun, Y. Li, S. Shervin, K.-H. Lee, V. Selvamanickam, J.-H. Ryou, High-efficiency flexible III-V photovoltaic solar cells based on single-crystal-like thin films directly grown on metallic tapes, Prog. Photovolt. Res. Appl., 2019, 27, 30-36

(7) K. Tanabe, A Review of Ultrahigh Efficiency III- V Semiconductor Compound Solar Cells: Multijunction Tandem, Lower Dimensional. Photonic Up/Down Conversion and Plasmonic Nanometallic Structures, Energy, 2009, 2, 504-530

(8) M. Yokohama, T. Yasuda, H. Takagi, H. Yamada, N. Fukuhara, M. Hata, M. Sugiyama, Y. Nakano, M. Takenaka, S. Takagi, Thin Body III-V Semiconductor-on-Insulator Metal-Oxide-Semiconductor Field-Effect Transistors on Si Fabicated Using Direct Wafer Bonding, Appl. Phys. Express, 2009, 2, 124501

(9) P. D. Ye, Main determinants for III-V metal-oxide-semiconductor field-effect transistors, J. Vac. Sci. Technol. A, 2008, 26(4), 697

(10) V. G. Dubrovskii, Theory of VLS Growth of Compound Semiconductors, Semiconductors and Semimetals, Chapter 1, Elsevier Inc, 2015, 93

(11) Y. B. Bolkhovityanov, O. P. Pchelyakov, GaAs epitaxy on Si substrates: modern status of research and engineering, Phys.-Usp., 2008, 51(5), 437

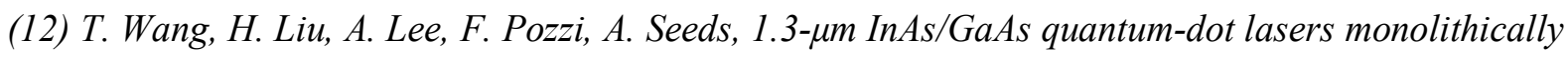
grown on Si substrates, Optics Express, 2011, 19(12), 11381-11386 
(13) E. Y. Chang, T.-H. Yang, G. Luo, C.-Y. Chang, A GeSi-buffer structure for growth of high-quality GaAs epitaxial layers on a Si substrate, J. Electron. Mater., 2005, 34(1), 23-26

(14) E.A. Fitzgerald, Y.-H. Xie, M. L. Green, D. Brasen, A. R. Kortan, J. Michel, Y.-J. Mii, B. E. Weir, Totally relaxed $G e_{x} S i_{1-x}$ layers with low threading dislocation densities grown on Si substrates, Appl. Phys. Lett., 1991, 59, 811

(15) V. K. Dixit, T. Ganguli, T. K. Sharma, R. Kumar, S. Porwal, V. Shukla, A. Ingale, P. Tiwari, A. K. Nath, Studies on MOVPE growth of GaP epitaxial layer on Si(001) substrate and effects of annealing, J. Cryst. Growth, 2006, 293(1), 5-13

(16) Y. Komatsu, K. Hosotani, T. Fuyuki, H. Matsunami, Heteroepitaxial Growth of InGaP on Si with InGaP/GaP Step-graded Buffer Layers, Jpn. J. Appl. Phys., 1997, 36, 5425

(17) T. Tsuji, H. Yonezu, N. Ohshima, Selective epitaxial growth of GaAs on Si with strained shortperiod superlattices by molecular beam epitaxy under atomic hydrogen irradiation, J. Vac. Sci. Technol. B, 2004, 22(3), 1428

(18) U. Gösele, Y. Bluhm, G. Kastner, P. Kopperschmidt, G. Krauter, R. Scholz, A. Schumacher, S. Senz, Q.-Y., Tong, L.-J., Huang, Y.-L. Chao, T. H. Lee, Fundamental issues in wafer bonding, J. Vac. Sci. Technol. A, 1999, 17(4), 1145

(19) T. Mårtensson, C. P. T. Svensson, B. A. Wacaser, M. W. Larsson, W. Seifert, K. Deppert, A. Gustafsson, L. R. Wallenberg, L. Samuelson, Epitaxial III-V Nanowires on Silicon, Nano Lett., 2004, 4(10), $1987-1990$

(20) J. Treu, T. Stettner, M. Watzinger, S. Morkötter, M. Döblinger, S. Matich, K. Saller, M. Bichler, G. Abstreiter, J. J. Finley, J. Stangl, G. Koblmüller, Lattice-Matched InGaAs-InAlAs Core-Shell Nanowires with Improved Luminescence and Photoresponse Properties, Nano Lett. 2015, 15(5), $3533-3540$

(21) J. C. Shin, A. Lee, P. K. Mohseni, D. Y. Kim, L. Yu, J. H. Kim, H. J. Kim, W. J. Choi, D. Wasserman, K. J. Choi and X. Li, Wafer-Scale Production of Uniform InAs $P_{1-y}$ Nanowire Array on Silicon for Heterogeneous Integration, ACS Nano, 2013, 7 (6), 5463-5471

(22) J. Wu, Y. Li, J. Kubota, K. Domen, M. Aagesen, T. Ward, A. Sanchez, R. Beanland, Y. Zhang, M. Tang, S. Hatch, A. Seeds and H. Liu, Wafer-Scale Fabrication of Self-Catalyzed $1.7 \mathrm{eV}$ GaAsP Core-Shell Nanowire Photocathode on Silicon Substrates, Nano Lett. 2014, 14(4), 2013-2018

(23) D. Saxena, N. Jiang, X. Yuan, S. Mokkapati, Y. Guo, H. H. Tan, C. Jagadish, Design and RoomTemperature Operation of GaAs/AlGaAs Multiple Quantum Well Nanowire Lasers, Nano Lett., 2016, 16(8), 5080-5086 
(24) T. Stettner, P. Zimmermann, B. Loitsch, M. Döblinger, A. Regler, B. Mayer, J. Winnerl, S. Matich, H. Riedl, M. Kaniber, G. Abstreiter, G. Koblmüller, J. J. Finley, Coaxial GaAs-AlGaAs core-multishell nanowire lasers with epitaxial gain control, Appl. Phys. Lett., 2016, 108, 011108

(25) K. Tomioka, J. Motohisa, S. Hara, K. Hiruma, T. Fukui, GaAs/AlGaAs Core Multishell NanowireBased Light-Emitting Diodes on Si, Nano Lett., 2010, 10(5), 1639-1644

(26) C. P. T. Svensson, T. Mårtensson, J. Trägårdh, C. Larsson, M. Rask, D. Hessman, L. Samuelson, J. Ohlsson, Monolithic GaAs/InGaP nanowire light emitting diodes on silicon, Nanotechnology, 2008, 19, 305201

(27) J. Huh, D. C. Kim, A. M. Munshi, D. L. Dheeraj, D. Jang, G. T. Kim, B. O. Fimland, H. Weman, Low frequency noise in single GaAsSb nanowires with self-induced compositional gradients, Nanotechnology, 2016, 27, 385703

(28) M. Sharma, E. Ahmad, D. Dev, J. Li, C. L. Reynolds Jr, Y. Liu, S. Iyer, Improved performance of GaAsSb/AlGaAs nanowire ensemble Schottky barrier based photodetector via in situ annealing, Nanotechnology, 2019, 30, 034005

(29) D. Ren, D. L. Dheeraj, C. Jin, J. S. Nilsen, J. Huh, J. F. Reinertsen, A. M. Munshi, A. Gustafsson, A. T. J. Van Helvoort, H. Weman, B. O. Fimland, New Insights into the Origins of Sb-Induced Effects on Self-Catalyzed GaAsSb Nanowire Arrays, Nano Lett., 2016, 16(2), 1201-1209

(30) M. J. L. Sourribes, I. Isakov, M. Panfilova, H. Liu, P. A. Warburton, Mobility Enhancement by Sbmediated Minimisation of Stacking Fault Density in InAs Nanowires Grown on Silicon, Nano Lett., 2014, 14(3), 1643-1650

(31) K. Tomioka, M. Yoshimura, T. Fukui, A III-V nanowire channel on silicon for high-performance vertical transistors, Nature, 2012, 488, 189-192

(32) J. J. Hou, N. Han, F. Wang, F. Xiu, S. P. Yip, A. T. Hui, T. F. Hung and J. C. Ho, Synthesis and Characterizations of Ternary InGaAs Nanowires by a Two-Step Growth Method for High-Performance Electronic Devices, ACS Nano, 2012, 6(4), 3624-3630

(33) A. Bengoechea-Encabo, F. Barbagini, S. Fernandez-Garrido, J. Grandal, J. Ristic, M. A. SanchezGarcia, E. Calleja, U. Jahn, E. Luna, A. Trampert, Understanding the selective area growth of GaN nanocolumns by MBE using Ti nanomasks, Journal of Crystal Growth, 2011, 325(1), 89-92

(34) X. Ji, X. Yang, W. Du, H. Pan, T. Yang, Selective-Area MOCVD Growth and Carrier-TransportType Control of InAs(Sb)/GaSb Core-Shell Nanowires, Nano Lett., 2016, 16(12), 7580-7587

(35) K. Tomioka, Selective-area growth of III-V nanowires and their applications, J. Mater. Res., 2011, 26(17), 2127-2141 
(36) K. Tomioka, T. Tanaka, S. Hara, K. Hiruma, T. Fukui, III-V Nanowires on Si Substrate: SelectiveArea Growth and Device Applications, IEEE Journal of selected topics in quantum electronics, 2011, 17(4)

(37) K. Yamano, K. Kishino, Selective area growth of InGaN-based nanocolumn LED crystals on AlN/Si substrates useful for integrated $\mu$-LED fabrication, Appl. Phys. Lett., 2018, 112(9), 091105

(38) D. Kohen, V. Tileli, C. Cayron, P. Faucherand, C. Morin, J. Dufourcq, S. Noël, M. Levis, A. Brioude, S. Perraud, Al catalyzed growth of silicon nanowires and subsequent in situ dry etching of the catalyst for photovoltaic application, Phys. Status Solidi A, 2011, 208(11), 2676-2680

(39) R. S. Wagner, W. C. Ellis, Vapor-liquid-solid mechanism of single-crystal growth, Appl. Phys. Lett., 1964, 4(5), 89-90

(40) M. E. Messing, K. Hillerich, J. Johansson, K. Deppert, K. A. Dick, The use of gold for fabrication of nanowire structures, Gold Bulletin, 2009, 42(3), 172-181

(41) Y. Zhang, J. Wu, M. Aagesen, H. Liu, III-V nanowires and nanowire optoelectronic devices, J. Phys. D., 2015, 48, 463001

(42) N. Li, T. Y. Tan, U. Gösele, Transition region width of nanowire hetero- and pn-junctions grown using vapor-liquid-solid processes, Appl. Phys. A, 2008, 90, 591-596

(43) K. Sarkar, M. Palit, P. Banerji, S. Chattopadhyay, N. N. Halder, P. Biswas, B. Nagabhusana and S. Chowdhurya, Silver catalyzed growth of $\mathrm{In}_{x} G a_{1-x}$ Asnanowires on $\mathrm{Si}(001)$ by metal-organic chemical vapour deposition, CrystEngComm, 2015, 17, 8519-8528

(44) C. Colombo, D. Spirkoska, M. Frimmer, G. Abstreiter, A. Fontcuberta i Morral, Ga-assisted catalyst-free growth mechanism of GaAs nanowires by molecular beam epitaxy, Phys. Rev. B, 2008, 77,155326

(45) S. G. Ghalamestani, M. Ek, M. Ghasemi, P. Caroff, J. Johansson, K. A. Dick, Morphology and composition controlled $G a_{x} I n_{1-x} S b$ nanowires: understanding ternary antimonide growth, Nanoscale, 2014, 6, 1086

(46) A. Berg, F. Lenrick, N. Vainorius, J. P. Beech, L. R. Wallenberg, M. T. Borgström, Growth parameter design for homogeneous material composition in ternary $G a_{x} I n_{1-x} P$ nanowires, Nanotechnology, 2015, 26, 435601

(47) K. A. Dick, J. Bolinsson, B. M. Borg, J. Johansson, Controlling the Abruptness of Axial Heterojunctions in III-V Nanowires: Beyond the Reservoir Effect, Nano Lett., 2012, 12(6), 3200-3206

(48) J. Motohisa, J. Noborisaka, S. Hara, M. Inari, T. Fukui, Catalyst-free growth of semiconductor nanowires by selective area MOVPE, AIP Conference Proceedings, 2005, 772, 877 
(49) G. Koblmüller, G. Abstreiter, Growth and properties of InGaAs nanowires on silicon, Phys. Status Solidi, 2013, 7(10)

(50) J. C. Shin, K. J. Choi, D. Y. Kim, W. Jun Choi, X. Li, Characteristics of Strain-Induced $\operatorname{In}_{x} G a_{1-x} A s$ Nanowires Grown on Si(111) Substrates, Cryst. Growth Des., 2012, 12, 2994-2998

(51) F. Glas, Critical dimensions for the plastic relaxation of strained axial heterostructures in freestanding nanowires, Physical Review B, 2006, 74, 121302

(52) L. Li, D. Pan, Y. Xue, X. Wang, M. Lin, D. Su, Q. Zhang, X. Yu, H. So, D. Wei, B. Sun, P. Tan, A. Pan, J. Zhao, Near Full-Composition-Range High-Quality GaAs ${ }_{1-x} S b_{s}$ Nanowires Grown by Molecular Beam Epitaxy, Nano Lett., 2017, 17(2), 622-630

(53) J. H. van der Merwe, Misfit dislocations in Epitaxy, Metallurgical and Materials Transactions A, 2002, 33(8), 2475-2483

(54) K. L. Kavanagh, Misfit dislocations in nanowire heterostructures, Semicond. Sci. Technol., 2010, 25, 024006

(55) M. de la Mata, C. Magen, P. Caroff, J. Arbiol. Atomic Scale Strain Relaxation in Axial Semiconductor III-V Nanowire Heterostructures, Nano Lett., 2014, 14(11), 6614-6620

(56) J. Grönqvist, N. Søndergaard, F. Boxberg, T. Guhr, S. Åberg,H. Q. Xu, Strain in semiconductor core/shell nanowires, J. Appl. Phys., 2009, 106, 053508

(57) D. Ferrand, J. Cibert, Strain in crystalline core-shell nanowires. European Physical Journal: Applied Physics, EDP Sciences, 2014, 67 (3), 30403

(58) L. Gagliano, M. Albani, M. A. Verheijen, E. P. A. M. Bakkers, L. Miglio, Twofold origin of straininduced bending in core-shell nanowires: the GaP/InGaP case, Nanotechnology, 2018, 29(31), 315703

(59) R. B. Lewis, P. Corfdir, H. Kupers, T. Flissikowski, O. Brandt, L. Geelhaar, Nanowires Bending over Backward from Strain Partitioning in Asymmetric Core-Shell heterostructures, Nano Lett., 2018 , 18(4), 2343-2350

(60) K. L. Kavanagh, I. Saveliev, M. Blumin, G. Swadener, H. E. Ruda, Faster radial strain relaxation in InAs-GaAs core-shell heterowires, Appl. Phys. Lett., 2012, 111, 044301

(61) S. A. Dayeh, W. Tang, F. Boioli, K. L. Kavanagh, H. Zheng, J. Wang, N. H. Mack, G. Swadener, J. Y. Huang, L. Miglio, K.-N. Tu, S. T. Picraux, Direct Measurement of Coherency Limits for Strain Relaxation in Heteroepitaxial Core/Shell Nanowires, Nano Lett., 2013, 13(5), 1869-1876

(62) J. Gronqvist, N. Sondergaard, F. Boxberg, T. Guhr, S. Aberg, H. Q. Xu, Strain in semiconductor core/shell nanowires, J. Appl. Phys., 2009, 106, 053508 
(63) A. Biermanns, T. Rieger, G. Bussone, U. Pietsch, D. Grutzmacher, M. I. Lepsa, Axial strain in GaAs/InAs core-shell nanowires, Appl. Phys. Lett., 2013, 102, 043109

(64) H. Zeng, X. Yu, H. A. Fonseka, J. A. Gott, M. Tang, Y. Zhang, G. Boras, J. Xu, A. M. Sanchez, H. Liu, Hybrid III-V/IV Nanowires: High- Quality Ge Shell Epitaxy on GaAs Cores, Nano Lett., 2018, 18(10), 6397-6403

(65) J. J. Tietjen, J. A. Amick, The preparation and properties of vapour-deposited epitaxial GaAs ${ }_{1-x} P_{x}$ using arsine and phosphine, J. Electrochem. Soc., 1966, 113, 724-728

(66) G. Priante, G. Patriarche, F. Oehler, F. Glas, J.-C. Harmand, Abrupt GaP/GaAs Interfaces in Self-Catalyzed Nanowires, Nano Lett., 2015, 15(9), 6036-6041

(67) N. N. Halder, A. Kelrich, S. Cohen, D. Ritter, Pure wurtzite GaP nanowires grown on zincblende GaP substrates by selective area vapor liquid solid epitaxy, Nanotechnology, 2017, 28, 465603

(68) H. S. Im, C. S. Jung, K. Park, D. M. Jang, Y. R. Lim, and J. Park, Band Gap Tuning of Twinned GaAsP Ternary Nanowires, J. Phys. Chem. C, 2014, 118(8), 4546-4552

(69) Y. Zhang, M. Aagesen, J. V. Holm, H. I. Jørgensen, J. Wu and H. Liu, Self-Catalyzed GaAsP Nanowires Grown on Silicon Substrates by Solid-Source Molecular Beam Epitaxy, Nano Lett., 2013, 13(8), 3897-3902

(70) Y. Zhang, J. Wu, M. Aagesen, J. Holm, S. Hatch, M. Tang, S. Huo, H. Liu, Self-Catalyzed Ternary Core-Shell GaAsP Nanowire Arrays Grown on Patterned Si substrates by Molecular Beam Epitaxy, Nano Lett., 2014, 14(8), 4542-4547

(71) J. Wu, A. Ramsay, A. M. Sanchez, Y. Zhang, D. Kim, F. Brossard, X. Hu, M. Benamara, M. E. Ware, Y. I. Mazur, G. J. Salamo, M. Aagesen, Z. Wang, H. Liu, Defect-Free Self-Catalyzed GaAs/GaAsP Nanowire Quantum Dots Grown on Silicon Substrate, Nano Lett., 2016, 16(1), 504-511

(72) I. Isakov, M. Panfilova, M. J. L. Sourribes, V. Tileli, A. E. Porter, P. A. Warburton, InAs $s_{1-x} P_{x}$ nanowires grown by catalyst-free molecular-beam epitaxy, Nanotechnology, 2013, 24(8), 085707

(73) J. H. Lee, M. W. Pin, S. J. Choi, M. H. Jo, J. C. Shin, S.-G. Hong, S. M. Lee, B. Cho, S. J. Ahn, N. W. Song, S.-H. Yi, Y. H. Kim, Electromechanical Properties and Spontaneous Response of the Current in InAsP Nanowires, Nano Lett., 2016, 16(11), 6738-6745

(74) A. I. Persson, M. T. Björk, S. Jeppesen, J. B. Wagner, L. R. Wallenberg, L. Samuelson, InAs $s_{1-x} P_{x}$ Nanowires for Device Engineering, Nano Lett., 2006, 6(3), 403-407 
(75) J. Trägårdh, A. I. Persson, J. B. Wagner, D. Hessman, L. Samuelson, Measurements of the band gap of wurtzite InAs I-x $_{x}$ nanowires using photocurrent spectroscopy, J. Appl. Phys., 2007, 101(12), 123701

(76) M. Tchernycheva, G. E. Cirlin, G. Patriarche, L. Travers, V. Zwiller, U. Perinetti, J.-C. Harmand, Growth and Characterization of InP Nanowires with InAsP Insertions, Nano Lett., 2007, 7(6), 15001504

(77) G. E.Cirlin, M. Tchernycheva, G. Patriarche, J.-C. Harmand, Effect of Postgrowth Heat Treatment on the Structural and Optical Properties of InP/InAsP/InP Nanowires, Semiconductors, 2012, 46(2), $175-178$

(78) L. Ma, X. Zhang, H. Li, H. Tan, Y. Yang, Y. Xu, W. Hu, X. Zhu, X. Zhuang, A. Pan, Bandgapengineered GaAsSb alloy nanowires for near-infrared photodetection at $1.31 \mu \mathrm{m}$, Semicond. Sci. Technol., 2015, 30(10), 105033

(79) J. Huh, H. Yun, D.-C. Kim, A. M. Munshi, D. L. Dheeraj, H. Kauko, A. T. J. van Helvoort, S. W. Lee, B.-O. Fimland, H. Weman, Rectifying Single GaAsSb Nanowire Devices Based on Self-Induced Compositional Gradients, Nano Lett., 2015, 15(6), 3709-3715

(80) D. Ren, J. Huh, D. L. Dheeraj, H. Weman, B.-O. Fimland, Influence of pitch on the morphology and luminescence properties of self-catalyzed GaAsSb nanowire arrays, Appl. Phys. Lett., 2016, 109, 243102

(81) X. Yu, L. Li, H. Wang, J. Xiao, C. Shen, D. Pan, J. Zhao, Two-step fabrication of self-catalyzed Ga-based semiconductor nanowires on Si by molecular-beam epitaxy, Nanoscale, 2016, 8, 10615 10621

(82) E. Ahmad, M. R. Karim, S. B. Hafiz, C. L. Reynolds, Y. Liu, S. Iyer, A Two-step Growth Pathway for High Sb Incorporation in GaAsSb Nanowires In The Telecommunication Wavelength Range, Sci. Rep., 2017, 7, 10111

(83) M. Sharma, M. R. Karim, P. Kasanaboina, J. Li, S. Iyer, Pitch-Induced Bandgap Tuning in SelfCatalyzed Growth of Patterned GaAsSb Axial and GaAs/GaAsSb Core-Shell Nanowires Using Molecular Beam Epitaxy, Cryst. Growth Des., 2017, 17(2), 730-737

(84) E. Alarcon-Llado, S. Conesa-Boj, X. Wallart, P. Caroff, A. Fontcuberta i Morral, Raman spectroscopy of self-catalyzed GaAs ${ }_{1-x} S b_{x}$ nanowires grown on silicon, Nanotechnology, 2013, 24(40), 405707 
(85) S. Conesa-Boj, D. Kriegner, X. Han, S. Plissard, X. Wallart, J. Stangl, A. Fontcuberta i Morral, P. Caroff, Gold-free Ternary III-V Antimonide Nanowire Arrays on Silicon: Twin-free Down To The First Bilayer, Nano Lett., 2014, 14(1), 326-332

(86) S. Plissard, K. A. Dick, X. Wallart, P. Caroff, Gold-free GaAs/GaAsSb heterostructure nanowires grown on silicon, Apll. Phys. Lett., 2010, 96, 121901

(87) A. Alhodaib, Y. J. Noori, P. J. Carrington, A. M. Sanchez, M. D. Thompson, R. J. Young, A. Krier, A. R. J. Marshall, Room-Temperature Mid-Infrared Emission from Faceted InAsSb Multi Quantum Wells Embedded in InAs Nanowires, Nano Lett., 2018, 18(1), 235-240

(88) W.-N. Du, X.-G. Yang, X.-Y. Wang, H.-Y. Pan, H.-M. Ji, S. Luo, T. Yang, Z.-G. Wang, The selfseeded growth of InAsSb nanowires on silicon by metal-organic vapour phase epitaxy, Journal of Crystal Growth, 2014, 396, 33-37

(89) E. A. Anyebe, Q. Zhang, Self-catalysed InAs $I_{-x} S b_{x}$ nanowires grown directly on bare Si substrates, Materials Research Bulletin, 2014, 60, 572-575

(90) Q. D. Zhang, E. A. Anyebe, R. Chen, H. Liu, A. M. Sanchez, M. K. Rajpalke, T. D. Veal, Z. M. Wang, Y. Z. Huang, H. D. Sun, Sb-Induced Phase Control of InAsSb Nanowires Grown by Molecular Beam Epitaxy, Nano Lett., 2015, 15(2), 1109-1116

(91) W. Du, X. Yang, H. Pan, X. Wang, H. Ji, S. Luo, X. Ji, Z. Wang, T. Yang, Two Different Growth Mechanisms for Au-Free InAsSb Nanowires Growth on Si Substrate, Cryst. Growth Des., 2015, 15(5), 2413-2418

(92) W. Du, X. Yang, H. Pan, X. Ji, H. Ji, S. Luo, X. Zhang, Z. Wang, T. Yang, Controlled-Direction Growth of Planar InAsSb Nanowires on Si Substrates without Foreign Catalysts, Nano Lett., 2016, 16(2), 877-882

(93) Q. D. Zhuang, H. Alradhi, Z. M. Jin, X. R. Chen, J. Shao, X. Chen, A. M. Sanchez, Y. C. Cao, J. Y. Liu, P. Yates, Optically efficient InAsSb nanowires for silicon-based mid-wavelength infrared optoelectronics, Nanotechnology, 2017, 28(10), 105710

(94) E. A. Anyebe, M. K. Rajpalke, T. D. Veal, C. J. Jin, Z. M. Wang, Q. D. Zhuang, Surfactant effect of antimony addition to the morphology of self-catalyzed InAs $I_{1-x} S b_{x}$ nanowires, Nano Res., 2015, 8(4), 1309-1319

(95) M. D. Thompson, A. Alhodaib, A. P. Craig, A. Robson, A. Aziz, A. Krier, J. Svensson, L.-E. Wernersson, A. M. Sanchez, A. R. J. Marshall, Low Leakage-Current InAsSb Nanowire Photodetectors on Silicon, Nano Lett., 2016, 16(1), 182-187 
(96) G. E. Cirlin, R. R. Reznik, I. V. Shtrom, A. I. Khrebtov, I. P. Soshnikov, S. A. Kukushkin, L. Leandro, T. Kasama, N. Akopian, AlGaAs and AlGaAs/GaAs/AlGaAs nanowires grown by molecular beam epitaxy on silicon substrates, J. Phys. D.: Appl. Phys., 2017, 50(48), 484003

(97) M. J. Tambe, S. K. Lim, M. J. Smith, L. F. Allard, S. Gradecak, Realization of defect-free epitaxial core/shell GaAs/AlGaAs nanowire heterostructures, Appl. Phys. Lett., 2008, 93, 151917

(98) L. V. Titova, T. B. Hoang, H. E. Jackson, L. M. Smith, J. M. Yarrison-Rice, Y. Kim, H. J. Joyce, H. H. Tan, C. Jagadish, Temperature dependence of photoluminescence from single core-shell GaAsAlGaAs nanowires, Appl. Phys. Lett., 2006, 89, 173126

(99) T. B. Hoang, L. V. Titova, J. M. Yarrison-Rice, H. E. Jackson, A. O. Govorov, Y. Kim, H. J. Joyce, H. H. Tan, C. Jagadish, L. M. Smith, Resonant Excitation and Imaging of Non-equilibrium Exciton Spins in Single Core-Shell GaAs-AlGaAs Nanowires, Nano Lett., 2007, 7(3), 588-595

(100) G. Koblmüller, B. Mayer, T. Stettner, G. Abstreiter, J. J. Finley, GaAs-AlGaAs core-shell nanowire lasers on silicon: invited review, Semicond. Sci. Technol., 2017, 32, 053001

(101) D. Saxena, S. Mokkapati, P. Parkinson, N. Jiang, Q. Gao, H. H. Tan, C. Jagadish, Optically pumped room-temperature GaAs nanowire lasers, Nature Photonics, 2013, 7, 963-968

(102) M. Heiss, Y. Fontana, A. Gustafsson, G. Wust, C. Magen, D. D. O’Regan, J. W. Luo, B. Ketterer, S. Conesa-Boj, A. V. Kuhlmann, J. Houel, E. Russo-Averchi, J. R. Morante, M. Cantoni, N. Marzari, J. Arbiol, A. Zunger, R. J. Warburton, A. Fontcuberta i Morral, Self-assembled quantum dots in a nanowire system for quantum photonics, Nature Materials, 2013, 12, 439-444

(103) C. Chen, S. Shehata, C. Fradin, R. LaPierre, C. Couteau, G. Weihs, Self-Directed Growth of AlGaAs Core-Shell Nanowires for Visible Applications, Nano Lett., 2007, 7(9), 2584-2589

(104) Z. H. Wu, M. Sun, X. Y. Mei, H. E. Ruda, Growth and photoluminescence characteristics of AlGaAs nanowires, Appl. Phys. Lett., 2004, 85(4), 657

(105) V. G. Dubrovskii, I. V. Shtrom, R. R. Reznik, Y. B. Samsonenko, A. I. Khrebtov, I. P. Soshnikov, S. Rouvimov, N. Akopian, T. Kasama, G. E. Cirlin, Origin of Spontaneous Core-Shell AlGaAs Nanowires Grown by Molecular Beam Epitaxy, Crys. Growth Des., 2016, 16(12), 7251-7255

(106) J. Guo, H. Hang, Y. Ding, Z. Ji, M. Liu, X. Ren, X. Zhang, Y. Huang, Growth of zinc blende GaAs/AlGaAs heterostructure nanowires on Si substrate by using AlGaAs buffer layers, Journal of Crystal Growth, 2012, 359, 30-34

(107) B. Loitsch, J. Winnerl, G. Grimaldi, J. Wierzbowski, D. Rudolph, S. Morkötter, M. Döblinger, G. Abstreiter, G. Koblmüller, J. J. Finley, Crystal Phase Quantum Dots in the Ultrathin Core of GaAsAlGaAs Core-Shell Nanowires, Nano Lett., 2015, 15(11), 7544-7551 
(108) C. P. Dietrich, A. Fiore, M. G. Thompson, M. Kamp, S. Höfling, GaAs integrated quantum photonics: Towards compact and multi-functional quantum photonic integrated circuits, Laser Photonics Rev., 2016, 10(6), 870-894

(109) R. Chen,T.-T. D. Tran, K. W. Ng, W. S. Ko, L. C. Chuang, F. G. Sedgwick, C. Chang-Hasnain, Nanolasers grown on silicon, Nature Photonics, 2011, 5, 170-175

(110) J. Tatebayashi, S. Kako, J. Ho, Y. Ota, S. Iwamoto, Y. Arakawa, Room-temperature lasing in a single nanowire with quantum dots, Nature Photonics, 2015, 9, 501-505

(111) J. J. Hou, F. Wang, N. Han, F. Xiu, S. P. Yip, M. Fang, H. Lin, T. F. Hung, J. C. Ho, Stoichiometric Effect on Electrical, Optical and Structural Properties of Composition-Tunable $\operatorname{In}_{x} G a_{1-x} A s$ Nanowires, ACS Nano, 2012, 6(10), 9320-9325

(112) J. C. Shin, D. Y. Kim, A. Lee, H. J. Kim, W. J. Choi, H.-S. Kim, K. J. Choi, Improving the composition uniformity of Au-catalyzed InGaAs nanowires on silicon, Journal of Crystal Growth, 2013, $372,15-18$

(113) J. C. Shin, K. H. Kim, H. Hu, K. J. Yu, J. A. Rogers, J.-M. Zuo, X. Li, Monolithically Grown $I_{x} G a_{1-x} A s$ Nanowire Array on Silicon Tandem Solar Cells with High Efficiency, IEEE Photonic Society, 2011, $24^{\text {th }}$ Annual Meeting

(114) J. C. Shin, K. H. Kim, K. J. Yu, H. Hu, L. Yin, C.-Z. Ning, J. A. Rogers, J.-M. Zuo, X. Li, In $\ln _{x} a_{1-}$ ${ }_{x}$ As Nanowires on Silicon: One-Dimensional Heterogeneous Epitaxy, Bandgap Engineering, and Photovoltaics, Nano Lett., 2011, 11(11), 4831-4838

(115) J. Treu, M. Speckbacher, K. Saller, S. Morkötter, M. Döblinger, X. Xu, H. Riedl, G. Abstreiter, J. J. Finley, G. Koblmüller, Widely tunable alloy composition and crystal structure in catalyst-free InGaAs nanowire arrays grown by selective area molecular beam epitaxy, Appl. Phys. Lett., 2016, 108(5), 053110

(116) S. Morkötter, S. Funk, M. Liang, M. Döblinger, S. Hertenberger, J. Treu, D. Rudolph, A. Yadav, J. Becker, M. Bichler, G. Scarpa, P. Lugli, I. Zardo, J. J. Finley, G. Abstreiter, G. Koblmüller, Role of microstructure on optical properties in high-uniformity $\operatorname{In}_{x} G a_{1-x} A s$ nanowire arrays: Evidence of a wider wurtzite band gap, Phys. Rev. B, 2013, 87, 205303

(117) A. Berg, S. Yazdi, A. Nowzari, K. Storm, V. Jain, N. Vainorius, L. Samuelson, J. B. Wagner, M. T. Borgström, Radial Nanowire Light-Emitting Diodes in the $\left(A l_{x} G a_{1-x}\right)_{y} I n_{1-y} P$ Material System, Nano Lett., 2016, 16(1), 656-662 
(118) P. Kivisaari, A. Berg, M. Karimi, K. Storm, S. Limpert, J. Oksanen, L. Samuelson, H. Pettersson, M. T. Borgström, Optimization of Current Injection in AlGaInP Core-Shell Nanowire Light-Emitting Diodes, Nano Lett., 2017, 17(6), 3599-3606

(119) X. Li, T.Shi, G. Liu, L.Wen, BuK. Zhou, Y. Wang, Absorption enhancement of GaInP nanowires by tailoring transparent shell thicknesses and its application in III-V nanowire/Si film two-junction solar cells, Optics Express, 2015, 23(19), 25316-15328

(120) S. E. H. Amiri, P. Ranga, D. Y. Li, F. Fan, C. Z. Ning, Growth of InGaP Alloy Nanowires with Widely Tunable Bandgaps on Silicon Substrates, Conference on Lasers and Electro-Optics, 2017, OSA Technical Digest, Optical Society of America, STh3I.4

(121) J. Tatebayashi, A. Lin, P. S. Wong, R. F. Hick, D. L. Huffaker, Visible light emission from selfcatalyzed GaInP/GaP core-shell double heterostructure nanowires on silicon, J. Appl. Phys., 2010, 108,034315

(122) A. Fakhr, Y. M. Haddara, R. R. LaPierre, Dependence of InGaP nanowire morphology and structure on molecular beam epitaxy growth conditions, Nanotechnology, 2010, 21(16), 165601

(123) D. Jacobsson, J. M. Persson, D. Kriegner, T. Etzelstorfer, J. Wallentin, J. B. Wagner, J. Stangl, L. Samuelson, K. Deppert, M. T. Borgström, Particle-assisted $G a_{x} I n_{1-x} P$ nanowire growth for designed bandgap structures, Nanotechnology, 2012, 23(24), 245601

(124) A. Berg, P. Caroff, N. Shahid, M. N. Lockrey, X. Yuan, M. T. Borgström, H. H. Tan, C. Jagadish, Growth and optical properties of $\operatorname{In}_{x} G a_{1-x} P$ nanowires synthesized by selective-area epitaxy, Nano Res., 2017, 10(2), 672-682

(125) G. Otnes, M. Heurlin, Xulu Zeng, M. T. Borgström, $I n_{x} G a_{1-x} P$ Nanowire Growth Dynamics Strongly Affected by Doping Using Diethylzinc, Nano Lett., 2017, 17(2), 702-707

(126) S. G. Ghalamestani, M. Ek, B. Gamjipour, C. Thelander, J. Johansson, P. Caroff, K. A. Dick, Demonstration of Defect-Free and Composition Tunable GaxIn $I_{1-x} S b$ Nanowires, Nano Lett., 2012, 12(9), 4914-4919

(127) H. Zhou, M. Pozuelo, R. F. Hicks, S. Kodambaka, Self-catalyzed vapour-liquid-solid growth of InP $P_{1-x} S b_{x}$ nanostructures, Journal of Crystal Growth, 2011, 319, 25-30

(128) H. B. Russell, A. N. Andriotis, M. Menon, J. B. Jasinski, A. Martinez-Garcia, M. K. Sunkara, Direct Band Gap Gallium Antimony Phosphide (GaSb $\left.P_{1-x}\right)$ Alloys, Sci. Rep., 2016, 6, 20822 
(129) L. Gagliano, M. Kruijsse, J. D. D. Schefold, A. Belabbes, M. A. Verheijen, S. Meuret, S. Koelling, A. Polman, F. Bechstedt, J. E. M. Haverkort, E. P. A. M. Bakkers, Efficient Green Emission from Wurtzite Al $_{x} I_{1-x} P$ Nanowires, Nano Lett., 2018, 18(6), 3543-3549

(130) B. Mayer, D. Rudolph, J. Schnell, S. Morkötter, J. Winnerl, J. Treu, K. Muller, G. Bracher, G. Abstreiter, G. Koblmüller, J. J. Finley, Lasing from individual GaAs-AlGaAs core-shell nanowires up to room temperature, Nature Communications, 2013, 4, 2931

(131) M. D. Birowosuto, A. Yokoo, G. Zhang, K. Tateno, E. Kuramochi, H. Taniyama, M. Takiguchi, M. Notomi, Movable high-Q nanoresonators realized by semiconductor nanowires on a Si photonic crystal platform, Nature Materials, 2014, 13, 279-285

(132) D. Ren, L. Ahtapodov, J. S. Nilsen, J. Yang, A. Gustafsson, J. Huh, G. J. Conibeer, A. T. J. van Helvoort, B.-O. Fimland, H. Weman, Single-Mode Near-Infrared Lasing in a GaAsSb-Based Nanowire Superlattice at Room Temperature, Nano Lett., 2018, 18(4), 2304-2310

(133) T. Stettner, A. Thurn, M. Döblinger, M. O. Hill, J. Bissinger, P. Schmiedeke, S. Matich, T. Kostenbader, D. Ruhstorfer, H. Riedl, M. Kaniber, L. J. Lauhon, J. J. Finley, G. Koblmüller, Tuning Lasing Emission toward Long Wavelengths in GaAs-(In,Al)GaAs Core-Multishell Nanowires, Nano Lett., 2018, 18(10), 6292-6300

(134) H. Kim, W.-J. Lee, A. C. Farrell, A. Balgarkashi, D. L. Huffaker, Telecom-Wavelength Bottomup Nanobeam Lasers on Silicon-on-Insulator, Nano Lett., 2017, 17, 5244-5250

(135) H. Kim, A. C. Farrell, P. Senanayake, W.-J. Lee, D. L. Huffaker, Monolithically Integrated InGaAs Nanowires on 3D Structured Silicon-on-Insulator as a New Platform for Full Optical Links, Nano Lett., 2016, 16, 1833-1839

(136) W.-J. Lee, H. Kim, J.-B. You, D. L. Huffaker, Ultracompact bottom-up photonic crystal lasers on silicon-on-insulator, Scientific Reports, 2017, 7, 9543

(137) Y. Zhang, H. Liu, Nanowires for High-Efficiency, Low-Cost Solar Photovoltaics, Crystals, 2019, $9(2), 87$

(138) R. Lin, S. V. Galan, H. Sun, Y. Hu, M. S. Alias, B. Janjua, T. K. Ng, B. S. Ooi, X. Li, Taperinginduced enhancement of light extraction efficiency of nanowire deep ultraviolet LED by theoretical simulations, Photonics Research, 2018, 6(5), 457-462

(139) Y. Zhang, A. M. Sanchez, M. Aagesen, S. Huo, H. A. Fonseka, J. A. Gott, D. Kim, X. Yu, X. Chen, J. Xu, T. Li, H. Zeng, G. Boras, H. Liu, Growth and Fabrication of High-Quality Single Nanowire Devices with Radial p-i-n Junctions, Small, 2019, 15(3), 1803684 
(140) J. V. Holm, H. I. Jørgensen, P. Krogstrup, J. Nygård, H. Liu, M. Aagesen, Surface-passivated GaAsP single-nanowire solar cells exceeding $10 \%$ efficiency grown on silicon, Nature Communications, 2013, 4, 1498

(141) J. J. Hou, F. Wang, N. Han, H. Zhu, K. W. Fok, W. C. Lam, S. P. Yip, T. F. Hung, J. E.-Y. Lee, J. C. Ho, Diameter dependence of electron mobility in InGaAs nanowires, Appl. Phys. Lett., 2013, 102(9), 093112

(142) O.-P. Kilpi, J. Svensson, J. Wu, A. R. Persson, R. Wallenberg, E. Lind, L.-E. Wernersson, Vertical InAs/InGaAs Heterostructure Metal-Oxide-Semiconductor Field-Effect Transistors on Si, Nano Lett., 2017, 17(10), 6006-6010 